\title{
A Review Paper on Heterogeneous Fenton Catalyst: Types of Preparation, Modification Techniques, Factors Affecting the Synthesis, Characterization, and Application in the Wastewater Treatment
}

\author{
Vijyendra Kumar, Titikshya Mohapatra, Sandeep Dharmadhikari, Prabir Ghosh* \\ Department of Chemical Engineering, National Institute of Technology Raipur, 492010 India
}

Received: 1 ${ }^{\text {st }}$ May 2019; Revised: 20th August 2019; Accepted: $23^{\text {rd }}$ August 2019;

Available online: $2^{\text {th }}$ February 2020; Published regularly: April 2020

\begin{abstract}
This comprehensive review focuses on the different factors, modification in the synthesis method, characterization and application of heterogeneous catalyst in the wastewater treatment based on the Fenton process. The present review highlights the different catalyst preparation methods like wet impregnation method, hydrothermal method, sol-gel method, precipitation method and their application to treat different recalcitrant organic chemicals. Major heterogeneous catalyst synthesis methods were discussed with their excellent workability. The importance of modification through physical and chemical method was also reported. Different catalyst, pollutants and optimum parametric conditions available in the literature along with some relevant studies are summarized. The effect of factors like $\mathrm{pH}$, calcination and some other modifiers on the synthesis and their efficiency in the wastewater treatment has been described. The important characterization of synthesized catalysts explaining their working efficiency has also been discussed. In the final section, the application of heterogeneous catalyst synthesized by different methods in the wastewater/effluent treatment has been investigated. The main aim of this review is to find out the influence of process parameters and catalytic method on degradation/decolorization of organic compounds present in industrial or synthetic wastewater. Copyright (C) 2020 BCREC Group. All rights reserved
\end{abstract}

Keywords: Catalytic Method; Fenton Process; Synthetic and Industrial Wastewater; Influence of Process Parameter; Modification of Catalyst Synthesis

How to Cite: Kumar, V., Mohapatra, T., Dharmadhikari, S., Ghosh, P. (2020). A Review Paper on Heterogeneous Fenton Catalyst: Types of Preparation, Modification Techniques, Factors Affecting the Synthesis, Characterization, and Application in the Wastewater Treatment. Bulletin of Chemical Reaction Engineering \& Catalysis, 15(1), 1-34 (doi:10.9767/bcrec.15.1.4374.1-34)

Permalink/DOI: https://doi.org/10.9767/bcrec.15.1.4374.1-34

\section{Introduction}

Many researchers are working on the treatment of wastewater generated from different industries like printing, dye [1], plastic, paint [2], textile [3], food, leather [4], petroleum [5], paper [6], cosmetics [7], distilleries [8], steel, rice mill,

* Corresponding Author.

E-mail: prbairg.che@nitrr.ac.in (P. Ghosh);

Tel.: +91 7773892359 sugar [9], fertilizers, pharmaceutical [10-13], sewage, oil extraction and refineries etc. [14-20]. These industries produce a huge amount of wastewater containing harmful organic compound that affects the environment directly or indirectly [21]. For instance, direct disposal of phenol and their derivatives into environment create serious issues for the living organism [2227]. In the same way, dyes are also intolerable and unacceptable due to poisonous and their re- 
fractory nature [28-30]. For the treatment of generated wastewater, various conventional and non-conventional methods like coagulation method [31], biological method [32], ion exchange method have been used, but among these methods, advanced oxidation technology (AOTs) is very effective comparatively [16,33]. During advanced oxidation reaction process, an oxidizing agent $(\cdot \mathrm{OH}$ radical) reacts with pollutant and degrades almost all the harmful recalcitrant compounds into $\mathrm{CO}_{2}, \mathrm{H}_{2} \mathrm{O}$, and inorganic salts [34]. Hydroxyl radical $(\cdot \mathrm{OH})$ is one of the strongest oxidizing oxidants $\left(\mathrm{E}^{0}=2.73 \mathrm{~V}\right)$. Hydroxyl radical generation is the basic principle of AOPs, produced by using hydrogen peroxide, UV-catalyst, $\mathrm{O}_{3}$, etc. and it mineralizes almost all organic chemicals [35].

The role of heterogeneous catalyst, preparation method, and process parameters are very important in the advanced oxidation processes. The applicability of catalysts has vast areas: chemical engineering, material science, surface
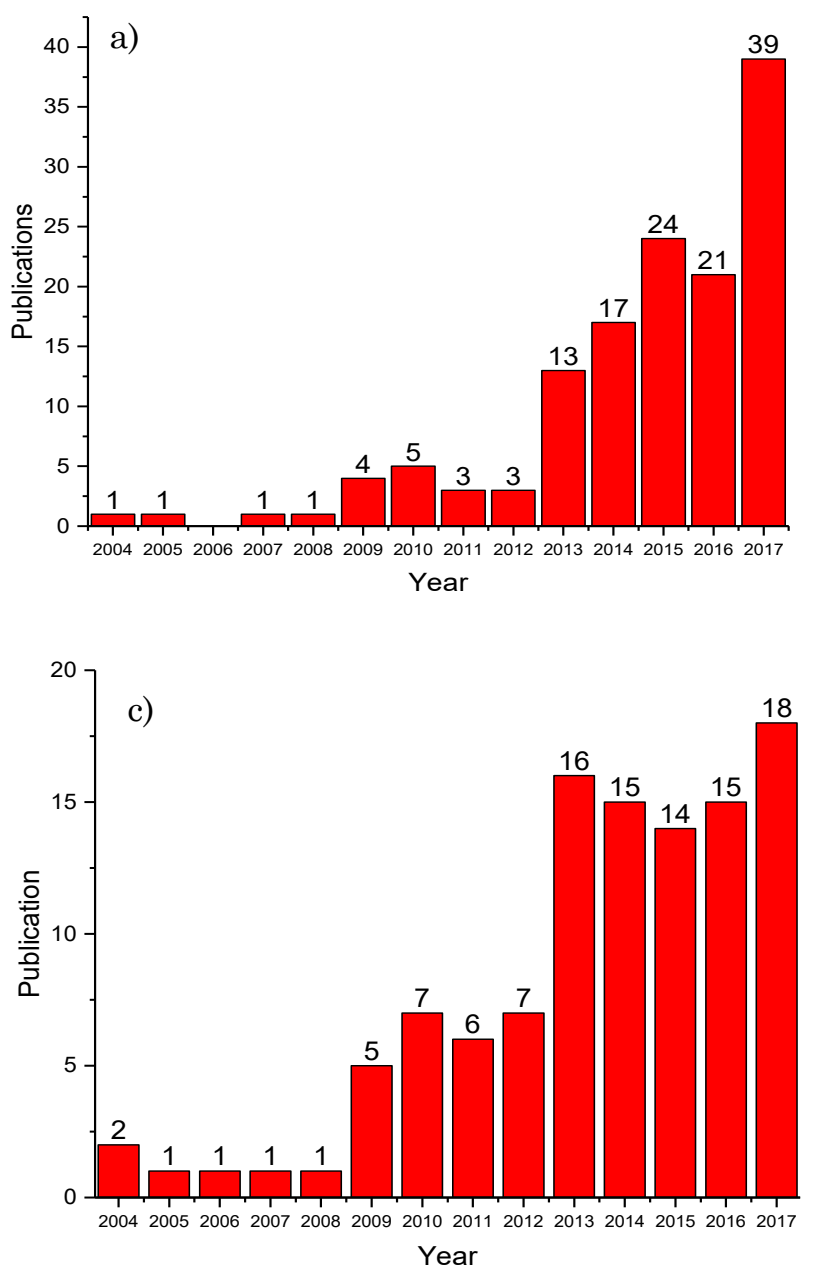

science, biochemistry, organic chemistry and inorganic chemistry [36]. Some examples of heterogeneous catalyst and pollutants are FeSBA-15/ $\mathrm{H}_{2} \mathrm{O}_{2}$ and Rhodamine B [28], $\mathrm{FeO}_{\mathrm{x}}, \mathrm{SiO}_{2}, \mathrm{TiO}_{2} / \mathrm{Ti} / \mathrm{H}_{2} \mathrm{O}_{2}$ and phenol [22], FeZSM-5 and Reactive Red 120 [29], $\mathrm{Fe}^{3+} / \mathrm{Cu}^{2+}$ grafted $\mathrm{ZnO}$ [37], copper loaded bentonite and Yellow FCF [38], Cu-Zeolites and 2(methylmercapto)-benzothiazole [39], Fe/clinoptilolite zeolite and phenol [23], $\mathrm{Cu}$ modified alkalinized $\mathrm{g}-\mathrm{C}_{3} \mathrm{~N}_{4}$ [40], $\mathrm{CuO} / \mathrm{Fe}_{2} \mathrm{O}_{3} / \mathrm{SBA}-15$ and $\mathrm{N}, \mathrm{N}$-diethyl-p-phenyl diamine degradation [41], $\mathrm{Fe}_{2} \mathrm{O}_{3} / \mathrm{ACF}$ and of acid red $\mathrm{B}$ [42], Fe/OMC and 4-chlorophenol [24], GO- $\mathrm{Fe}_{2} \mathrm{O}_{3}$ [43], HPW-Fe-Bent and methyl orange[44], $\mathrm{Fe} / \mathrm{meso}-\mathrm{Al}_{2} \mathrm{O}_{3}$ and phenol [45], $\mathrm{Fe}(\mathrm{III})-\mathrm{SiO}_{2}$ and polyacrylamide [46], Fe(III)HY[47], Fe-doped $\mathrm{TiO}_{2}$ and carboxylic acids [48], $\mathrm{Cs}_{\mathrm{x}} \mathrm{H}_{3-\mathrm{x}} \mathrm{PW}_{12} \mathrm{O}_{40}$ [49], CuFeZSM-5 and Rhodamine 6G [50], Bi-doped goethitehematite and actual pesticide [51], $\mathrm{Ag}_{2} \mathrm{O}-\mathrm{ZnO}$ [52], Schwertmannite and phenol [26], Fe-
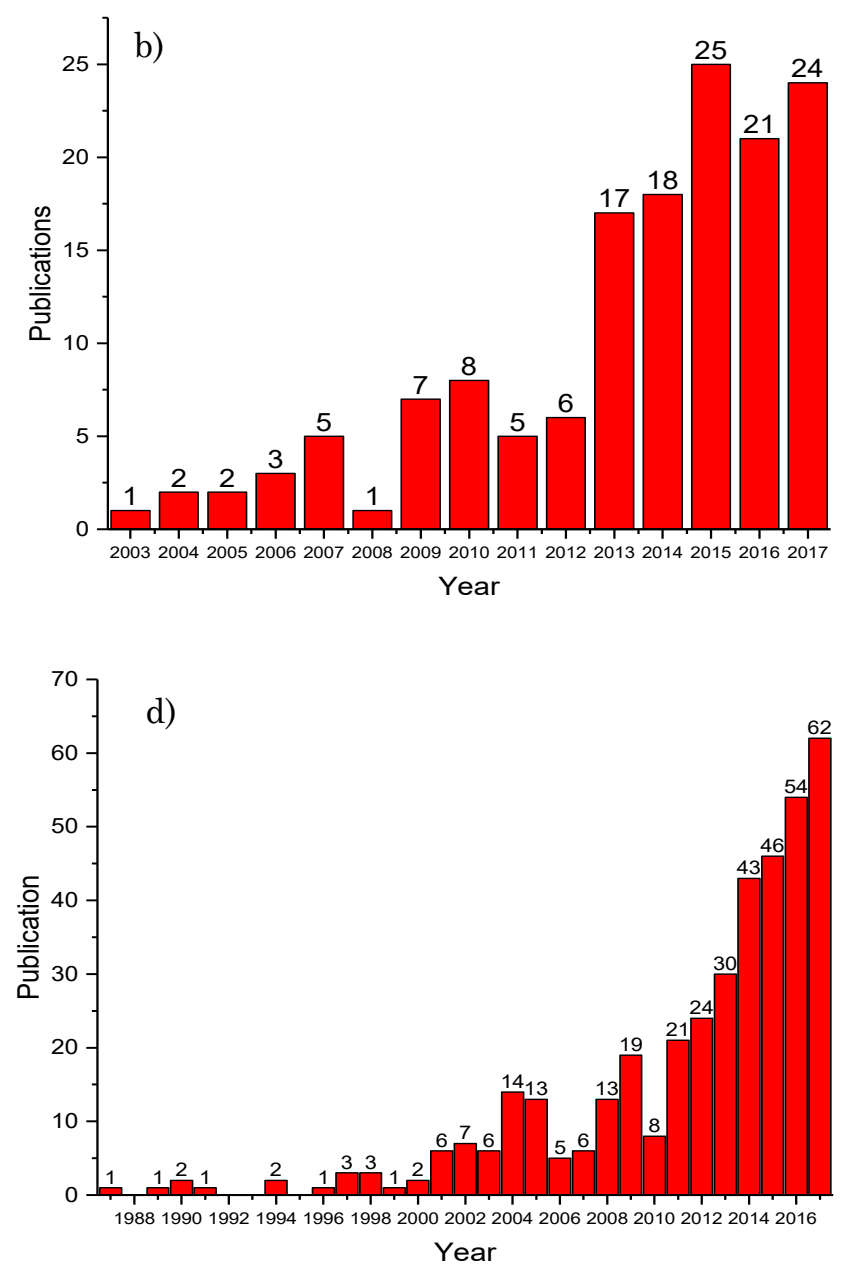

Figure 1. (a, b, c, and d) A number of research papers related to the application of the hydrothermal method, wet impregnation method, sol-gel, precipitation and co-Precipitation method in Fenton process to the wastewater treatment. Source: Scopus (December 2017) 
titanate [53], Ferric giniite [54], $\mathrm{Fe}_{3} \mathrm{O}_{4}-\mathrm{GO}$ and phenol [27], $\mathrm{BiFeO}_{3}-\mathrm{g}-\mathrm{C}_{3} \mathrm{~N}_{4}$ and lignin [55], $\mathrm{MoS}_{2} / \mathrm{Fe}_{3} \mathrm{O}_{4}$ [56], $\mathrm{Ag}$ loaded $\mathrm{CoFe}_{2} \mathrm{O}_{4} / \mathrm{Fe}_{2} \mathrm{O}_{3}$ and R6G [57]. Figure 1 (a, b, c, and d) shows the number of research papers related to the appli-

Hydrothermal Method

Wet Impregnation Method

Sol-Gel Method

Precipitation Method

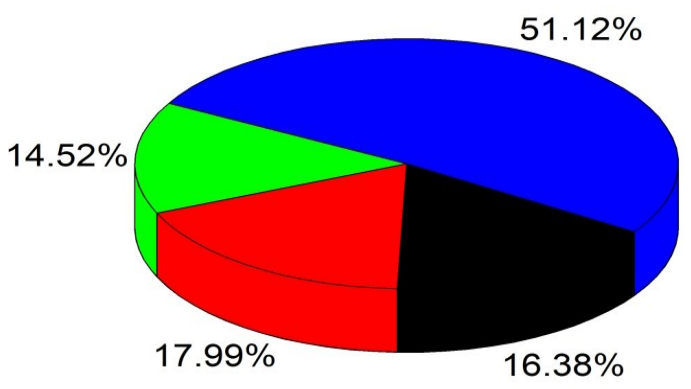

Figure 2. Three-dimensional graphical representation of published papers on different catalyst preparation methods in the Fenton process. cation of the hydrothermal method [57-59], wet impregnation method [22,28,29], sol-gel method $[4,17,60]$, precipitation and co-precipitation method [61-63] with the Fenton process in the field of wastewater treatment. Figure 2 depicts the pie chart of published papers of four different catalyst preparation methods used in the Fenton process. Figure 2 also displays that the most commonly used methods in the Fenton process are precipitation and co-precipitation method (51.12\%) till December 2017.

The characterization of any synthesized catalyst is necessary to reveal the scientific reason behind their efficiency. For instance, BET surface area investigates the specific surface area and available pore volume in the catalyst. Fourier transform infrared spectroscopy (FTIR) is used to obtain the idea about available functional group present in a catalyst with a wide range of intensity through absorbance or transmittance. Materials like metals, salts, semiconductors, minerals, and many organic-inorganic chemicals can form a crystal. X-ray diffraction (XRD) is helpful to know about the development of catalyst scientifically by measuring the

a)

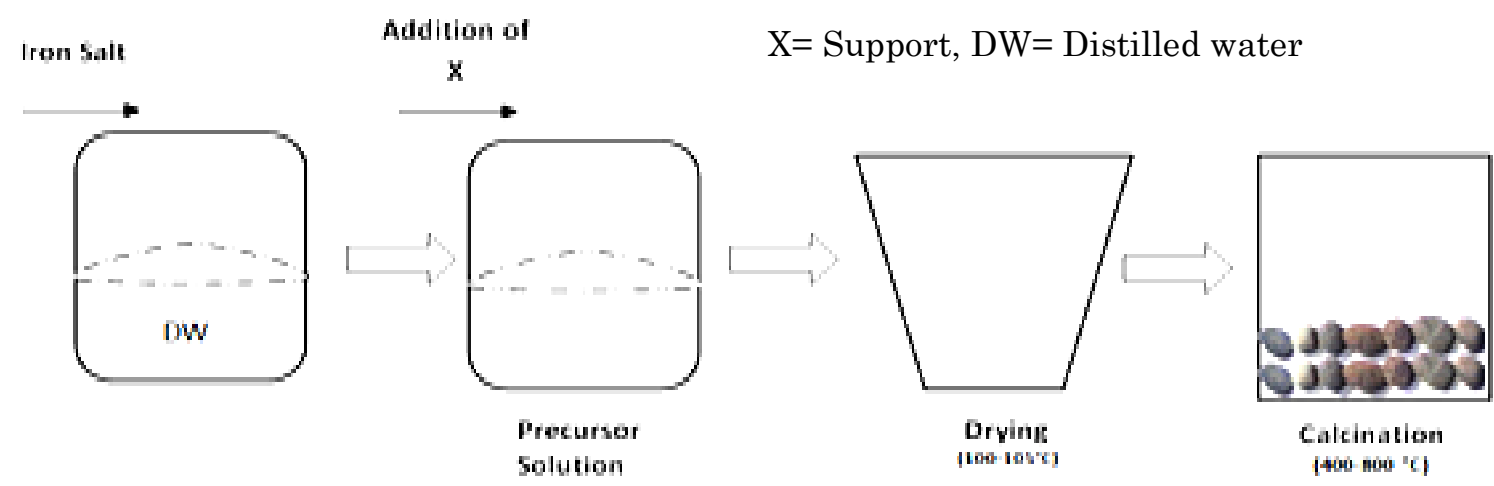

b)

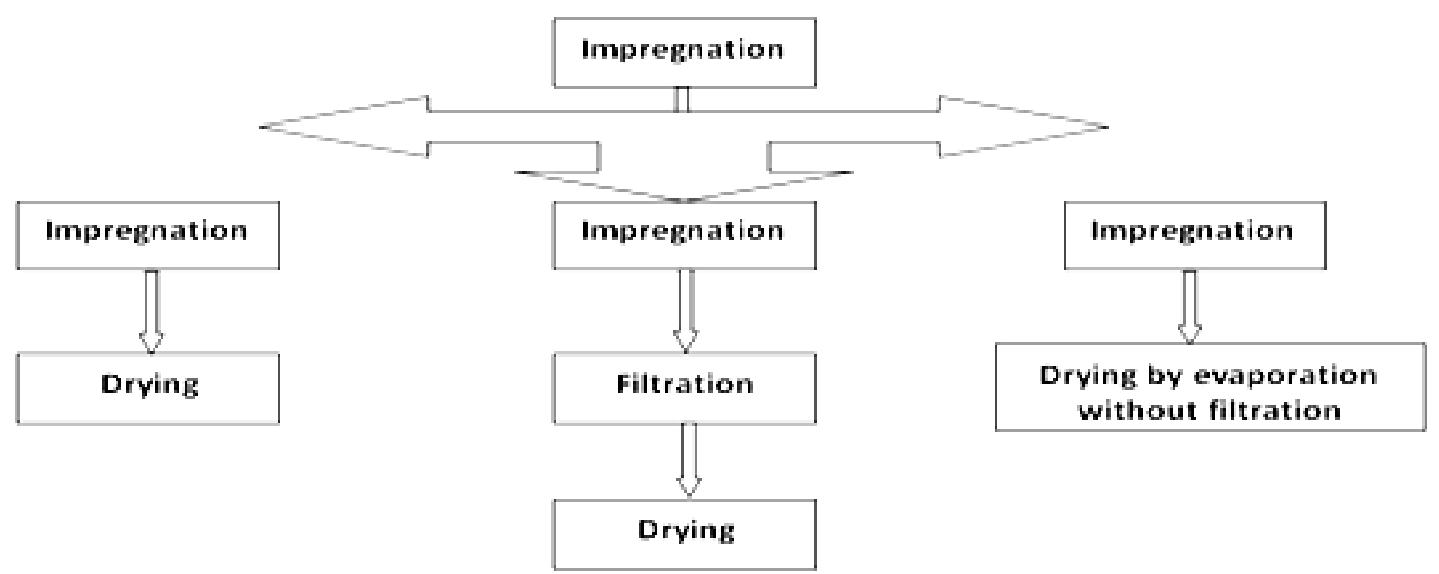

Figure 3. (a) Schematic diagram of wet impregnation process. (b) Possibilities of wet impregnation process. 
intensities and angle through the diffracted beam from the material. Scanning Electron Microscopy (SEM) produces images of the sample through an incident beam of electrons on the surface of the material. The SEM can significantly achieve the resolution better than $1 \mathrm{~nm}$. Thermo Gravimetric Analysis (TGA) is a technique to get information about the chemical and physical phenomena like thermal decomposition, chemisorption, solid-gas reaction and desorption, adsorption and phase transition, respectively. Temperature Programmed Reduction (TPR) profile characterization is especially useful for a heterogeneous catalyst to find the most significant oxidation/reduction condition. Raman spectroscopy observes the rotational, vibration and low frequency mode of any structural fingerprint of molecules identified by the same.

The modification of any catalyst is needed to enhance their respective characteristic and properties in the area of wastewater treatment. This can be possible with the physical and chemical modification like changing of morphology or using some modifiers. Many researchers investigated the influence of calcination temperature, acid and alkali treatment on heterogeneous catalyst.

The objective of the present review is to explore the recent development of heterogeneous catalysts on the Fenton process. Major catalyst preparation methods like wet impregnation, hydrothermal, sol-gel method, and their applications are studied in this review paper. The effect of $\mathrm{pH}$ and calcinations are also important in wastewater treatment. Present literature review reports the article published in last two decades on the heterogeneous catalyst for the

(a)

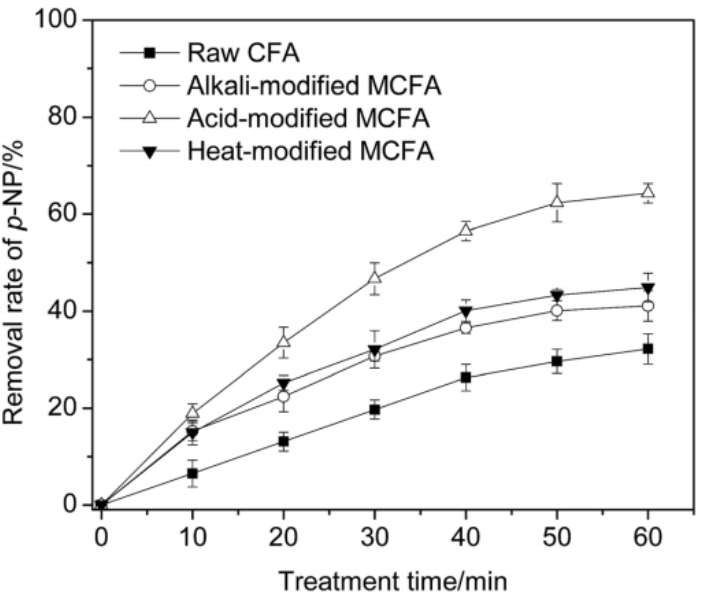

treatment of recalcitrant, harmful organic compound and real wastewater along with reaction conditions and catalytic performances. Figure 1 and Figure 2 demonstrate the number of papers published in last 2 decades.

Present review is aimed at finding out the most significant and widely used catalyst preparation methods for the treatment of synthetic and industrial wastewater in Fenton's process. Authors also demonstrated the simplification and possible process of heterogeneous catalyst preparation methods. In addition, various parametric conditions are also tabulated to compare with each other. There are many important parameters to know about the catalyst preparation methods with respect to ageing, calcination temperature, solubility and washing $(\mathrm{pH})$ as these parameters decide about the size, structure, optical, magnetic property, dimensions, homogeneous-heterogeneous nucleation and their efficiency. Present review has been focused for the same. Stability and recyclability which are important property for any useful, effective and significant catalyst has also been discussed in this work. Modification of catalyst during preparation can change their property and characteristics which have been described along with modification techniques. Factors such as $\mathrm{pH}$, calcination, modifier or RPM affecting the synthesis of catalyst with respect to the surface area, pore volume, diameter of particles have been elaborated in the present review. The characterizations of synthesized catalyst are necessary to know the scientific reasons behind the outcomes which may be the next step for new research. Variety of research on synthesized catalyst have been used in Fenton's process have been discussed

(b)

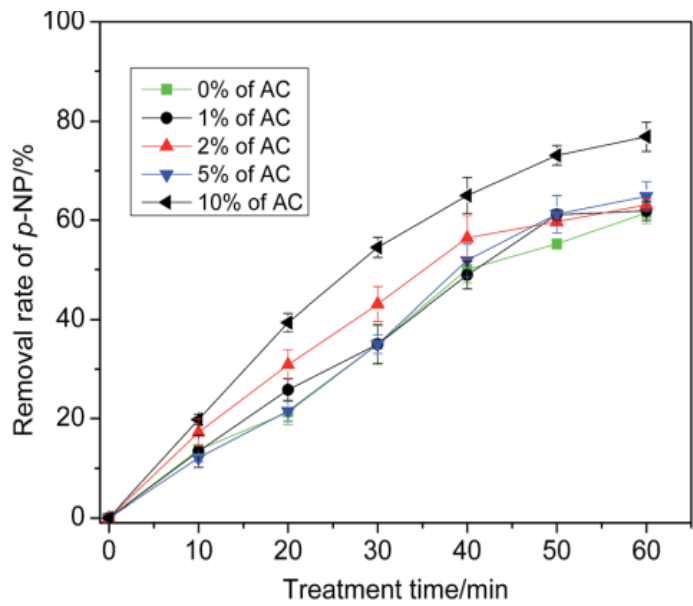

Figure 4. (a) Effect of acid, alkali and heat treatment on modification/activation of the catalyst (b) Influence of modifier on the enhancement of catalytic efficiency [68] (CFA: Coal fly ash, MCFA: Modified coal fly ash) (Reprinted with permission from Ref. [68] copyright from Royal Society of Chemistry) 
Table 1. Catalyst prepared by WI method, optimum conditions and their performances

\begin{tabular}{|c|c|c|c|c|c|c|c|c|c|}
\hline \multirow{2}{*}{$\begin{array}{l}\text { Pollutants } \\
\text { treated }\end{array}$} & \multirow{2}{*}{$\begin{array}{l}\text { Catalyst used in } \\
\text { WI method }\end{array}$} & \multicolumn{4}{|c|}{ Optimum Conditions } & \multicolumn{3}{|c|}{$\begin{array}{c}\text { Maximum } \\
\text { \% Degradation }\end{array}$} & \multirow[b]{2}{*}{ Ref. } \\
\hline & & $\begin{array}{l}\text { Initial } \\
\text { Conc. } \\
\text { (ppm) }\end{array}$ & $\mathrm{pH}$ & $\begin{array}{l}\text { Catalyst } \\
\text { dose } \\
(\mathrm{ppm})\end{array}$ & $\begin{array}{l}\mathrm{H}_{2} \mathrm{O}_{2} \\
(\mathrm{mM})\end{array}$ & $\begin{array}{l}\text { COD } \\
(\%)\end{array}$ & $\begin{array}{c}\text { Color } \\
(\%)\end{array}$ & $\begin{array}{l}\text { TOC } \\
(\%)\end{array}$ & \\
\hline $\begin{array}{l}\text { Rhodamine B } \\
\text { dye }\end{array}$ & Fe-SBA-15 & 10 & 3 & 15 & - & 97.7 & 35.1 & 97 & {$[28]$} \\
\hline Red 120 & Fe-ZSM-5 & 10 & 3 & 0.1 & - & - & 98 & - & [29] \\
\hline Phenol & $\begin{array}{l}\text { Fe/Faujasite Y } \\
\text { zeolite }\end{array}$ & $\begin{array}{c}0.8-88 \\
\mathrm{mg} / \mathrm{L}\end{array}$ & 5.5 & $1 \mathrm{~g} / \mathrm{L}$ & 0.007 & & 100 & & [65] \\
\hline P-nitrophenol & ACFA & 25 & 3 & $5.5 \mathrm{mM} / \mathrm{L}$ & 5 & 85.6 & - & - & [69] \\
\hline Methylene Blue & $\begin{array}{l}\text { Kiesel- } \\
\text { guhr/Fe } \mathrm{Fe}_{2} / \mathrm{TiO}_{2}\end{array}$ & $2.9 \times 10^{-5}$ & 3 & 2 & 13.7 & - & - & 65.5 & [70] \\
\hline Bisphenol A & Fe-GS & 100 & 3 & 20 & $530 \mathrm{mg} / \mathrm{L}$ & 40 & & 65 & [71] \\
\hline $\begin{array}{l}\text { N, N-diethyl-p- } \\
\text { phenyl diamine }\end{array}$ & $\mathrm{Cu} / \mathrm{Fe}-\mathrm{SBA}-15$ & 100 & 3.1 & 10 & 8 & 83 & & 80 & [41] \\
\hline Phenol & $\mathrm{Fe}-\mathrm{Cu}$ allophane & $5 \times 10^{-5} \mathrm{M}$ & 3 & - & 3.5 & 80 & - & - & [72] \\
\hline Crystal violet & Fe/Clay & $0.02 \mathrm{~g} / \mathrm{L}$ & 7 & 0.75 & 2 & 99 & 99 & - & [73] \\
\hline Orange II & $\begin{array}{l}\mathrm{FeCl}_{3} \text { and } \\
\mathrm{K}_{4}\left[\mathrm{Fe}(\mathrm{CN})_{6}\right]\end{array}$ & $0.2 \mathrm{mM} / \mathrm{L}$ & 3 & $6.5 \mathrm{~g} / \mathrm{L}$ & 9 & & 100 & 60 & [74] \\
\hline Astrazon Blue & $\mathrm{Fe} / \mathrm{ACs}$ & $\begin{array}{c}828 \mathrm{mg} \\
\mathrm{O}_{2} / \mathrm{L}\end{array}$ & 3 & $1.76 \mathrm{~g} / \mathrm{L}$ & 3.52 & 35.5 & 62.7 & 39.9 & {$[75]$} \\
\hline $\begin{array}{l}\text { Coking } \\
\text { wastewater }\end{array}$ & $\mathrm{Cu} / \mathrm{Fe} / \mathrm{TiO}_{2} / \mathrm{Al}_{2} \mathrm{O}_{3}$ & - & - & $1 \mathrm{~g} / \mathrm{L}$ & 14.68 & 65 & - & 68 & [76] \\
\hline Congo Red dye & Cu/zeolite Y & $\begin{array}{c}0.143 \\
\mathrm{mM}\end{array}$ & 7 & $1 \mathrm{~g} / \mathrm{L}$ & $\begin{array}{c}52.24 \\
\mathrm{mM}\end{array}$ & 93.58 & 95.34 & 79.52 & [77] \\
\hline Acid Red B & $\mathrm{Fe}_{2} \mathrm{O}_{3} / \mathrm{ACF}$ & 100 & 3 & 175 & $334 \mathrm{mg} / \mathrm{L}$ & - & 100 & 43 & [42] \\
\hline Clofibric acid & $\mathrm{Pd} / \mathrm{FeOOH}$ & 25 & 3.3 & $2 \mathrm{~g} / \mathrm{L}$ & 0.5 & - & - & 82 & [78] \\
\hline Phenol & $\mathrm{Fe}_{2} \mathrm{O}_{3} / \mathrm{Al}_{2} \mathrm{O}_{3}$ & $5 \mathrm{~g} / \mathrm{L}$ & 2 & $1.8 \mathrm{~g} / \mathrm{L}$ & 0.54 & - & - & 80 & [79] \\
\hline Phenol & $\mathrm{Fe}_{2} \mathrm{O}_{3} / \mathrm{Y}-\mathrm{Al}_{2} \mathrm{O}_{3}$ & $1 \mathrm{~g} / \mathrm{L}$ & 4.5 & $2.5 \mathrm{~g} / \mathrm{L}$ & $\begin{array}{c}0.15 \\
\mathrm{~mol} / \mathrm{L}\end{array}$ & 92 & - & - & [80] \\
\hline P-nitrophenol & $\mathrm{CuO} / \mathrm{Al}_{2} \mathrm{O}_{3}$ & 50 & 6 & 4 & 25 & - & - & 68 & [81] \\
\hline Acid scarlet 3R & $\mathrm{CuO} / \mathrm{SiO}_{2}$ & $0.4 \mathrm{~g} / \mathrm{L}$ & 3.5 & $6 \mathrm{~g} / \mathrm{L}$ & 34 & 90 & 97 & - & [82] \\
\hline
\end{tabular}


Table 1.... (continued)

\begin{tabular}{|c|c|c|c|c|c|c|c|c|c|}
\hline \multirow[b]{2}{*}{$\begin{array}{l}\text { Pollutants } \\
\text { treated }\end{array}$} & \multirow{2}{*}{$\begin{array}{l}\text { Catalyst used in } \\
\text { WI method }\end{array}$} & \multicolumn{4}{|c|}{ Optimum Conditions } & \multicolumn{3}{|c|}{$\begin{array}{c}\text { Maximum } \\
\text { \% Degradation }\end{array}$} & \multirow[b]{2}{*}{ Ref } \\
\hline & & $\begin{array}{l}\text { Initial } \\
\text { Conc. } \\
\text { (ppm) }\end{array}$ & $\mathrm{pH}$ & $\begin{array}{c}\text { Catalyst } \\
\text { dose } \\
(\mathrm{ppm})\end{array}$ & $\begin{array}{l}\mathrm{H}_{2} \mathrm{O}_{2} \\
(\mathrm{mM})\end{array}$ & $\begin{array}{l}\text { COD } \\
(\%)\end{array}$ & $\begin{array}{l}\text { Color } \\
(\%)\end{array}$ & $\begin{array}{l}\text { TOC } \\
(\%)\end{array}$ & \\
\hline 4-chlorophenol & $\mathrm{Fe} / \mathrm{OMC}$ & 100 & 3 & 40 & 6.6 & 96.1 & & 47.4 & {$[24]$} \\
\hline Black 5 dye & $\mathrm{Fe} / \mathrm{RHA}$ & 100 & 3 & $0.5 \mathrm{~g} / \mathrm{L}$ & 4 & 59.71 & 89.18 & & [83] \\
\hline Reactive Black 5 & Fe-AN & 50 & 2.5 & 1 & 16 & - & 99 & - & [84] \\
\hline $\begin{array}{l}6 \text {-Nitryl } \\
\text { wastewater }\end{array}$ & $\mathrm{Fe}-\mathrm{Cu}-\mathrm{Y}$ & $\begin{array}{l}12610 \\
\mathrm{mg} / \mathrm{L}\end{array}$ & 7 & 7.5 & $3 \mathrm{~mL}$ & 97 & - & - & [85] \\
\hline $\begin{array}{l}\text { Diisopropanola- } \\
\text { mine (DIPA) }\end{array}$ & $\mathrm{Fe} / \mathrm{TiO}_{2}$ & 100 & 1.77 & - & - & 80 & - & - & [86] \\
\hline Acid Green 25 & Fe-IC & $50 \mathrm{mg} / \mathrm{L}$ & 3 & 1.25 & 6.7 & - & 95 & - & [87] \\
\hline Reactive black 5 & Fe-areca nut & $50 \mathrm{mg} / \mathrm{L}$ & 2.5 & 1 & 16 & - & 99 & - & [84] \\
\hline Methyl orange & NdFeB-AC-FC & $20 \mathrm{mg} / \mathrm{L}$ & 3 & $10 \mathrm{~g} / \mathrm{L}$ & 0.6 & 97.8 & - & - & [88] \\
\hline $\begin{array}{l}\text { Rhodamine B and } \\
\text { 4-nitrophenol }\end{array}$ & $\mathrm{GO}-\mathrm{Fe}_{2} \mathrm{O}_{3}$ & 100 & 2 & $0.2 \mathrm{~g} / \mathrm{L}$ & 10 & - & 99 & 76 & [43] \\
\hline Phenol & Fe/clinoptilolite & $100 \mathrm{mg} / \mathrm{L}$ & 3.5 & 5 & $\begin{array}{c}5.876 \\
\mathrm{~mm}\end{array}$ & 70 & - & - & [89] \\
\hline Phenol & $\mathrm{Fe} / \mathrm{NH} 4 \mathrm{Y}$ & $100 \mathrm{mg} / \mathrm{L}$ & - & 1 & $1.65 \mathrm{~g} / \mathrm{L}$ & - & 96 & - & [90] \\
\hline 4-Chlorophenol & $\mathrm{Fe}_{3} \mathrm{O}_{4} / \mathrm{CeO}_{2}$ & $0.78 \mathrm{mM}$ & 3 & $2 \mathrm{~g} / \mathrm{L}$ & $30 \mathrm{~mm}$ & & $\begin{array}{l}954- \\
\mathrm{CP}\end{array}$ & 66 & {$[91]$} \\
\hline fparaquat & $\begin{array}{l}\text { Modified activat- } \\
\text { ed carbon }\end{array}$ & $25 \mathrm{mg} / \mathrm{L}$ & 3 & 1 & 12.5 & 92 & - & - & {$[92]$} \\
\hline 2,4-dichlorophenol & Fe/SBA-15 & $100 \mathrm{mg} / \mathrm{L}$ & 3 & $0.05 \mathrm{~g}$ & $1 \mathrm{~g} / \mathrm{L}$ & - & - & 60 & {$[93]$} \\
\hline Polyacrylamide & $\mathrm{Fe}(\mathrm{III})-\mathrm{SiO}_{2}$ & $100 \mathrm{mg} / \mathrm{L}$ & 6.8 & $1.0 \mathrm{~g} / \mathrm{L}$ & $200 \mathrm{mg} / \mathrm{L}$ & & $\begin{array}{c}94 \\
\text { PAM }\end{array}$ & 60 & {$[46]$} \\
\hline Phenol & $\begin{array}{l}\text { Clinoptilolite } \\
\text { zeolite }\end{array}$ & $100 \mathrm{mg} / \mathrm{L}$ & 3.25 & $15 \mathrm{~g} / \mathrm{L}$ & $20 \mathrm{mg} / \mathrm{L}$ & 91.6 & $\begin{array}{c}98 \\
\text { phenol }\end{array}$ & - & [23] \\
\hline Orange G & $\mathrm{Fe}_{3} \mathrm{O}_{4} / \mathrm{CeO}_{2}$ & $50 \mathrm{mg} / \mathrm{L}$ & 2.5 & 2 & $26 \mathrm{mM}$ & - & $\begin{array}{l}98.2 \\
\mathrm{OG}\end{array}$ & - & {$[94]$} \\
\hline Sulfamethazine & $\mathrm{Fe}_{3} \mathrm{O}_{4}-\mathrm{Mn}_{3} \mathrm{O}_{4} / \mathrm{Gr}$ & $20 \mathrm{mg} / \mathrm{L}$ & 3 & $0.5 \mathrm{~g} / \mathrm{L}$ & 6 & - & $98 \mathrm{smt}$ & - & {$[95]$} \\
\hline 2-chlorophenol & $\begin{array}{l}\text { Mesostructured } \\
\text { silicananoparticle }\end{array}$ & $50 \mathrm{mg} / \mathrm{L}$ & 5.11 & $0.40 \mathrm{~g} / \mathrm{L}$ & $0.14 \mathrm{mM}$ & 99.9 & - & - & {$[96]$} \\
\hline
\end{tabular}


in this review to know about the efficiency of the different processes with different parametric conditions.

\section{Catalyst: Types of Synthesis}

\subsection{Wet Impregnation Method}

Wet impregnation (WI), also called dry impregnation or capillary impregnation is a frequently used method for the synthesis of heterogeneous catalysts. In this technique, the metal precursor is dissolved in an aqueous solution. Then the same pore volume of metal-containing solution is added into a catalyst support. Due to capillary action, solution goes into the pores. After this, drying and calcinations are done to remove the volatile component. It is a process of enhancement of dispersion of active phase on support [36]. Figure 3 (a and b) demonstrates the flow diagram of WI method and possible processes.

Garcia et al. studied the catalyst preparation from Rose stems impregnated with iron oxide by wet impregnation method and found the excellent surface area. Significant color (98.33\%) was removed only in 20 min with $3 \%$ wt of Fe [64]. Alam et al. reported that the synthesis of $\mathrm{Fe}^{3+} / \mathrm{Cu}^{2+}$ over $\mathrm{ZnO}$ can be utilized for the degradation of Rhodamine $\mathrm{B}$ (RhB), 4-nitrophenol (4-NP) and Paracetamol. The prepared catalyst is very sensitive to visible light but the iron grafted $\mathrm{ZnO}$ exhibited higher activity than copper grafted zinc oxide [37]. Faujasite $\mathrm{Y}$ zeolite impregnated with $\mathrm{Fe}^{3+}$ showed excellent potential to degrade micropollutants like carbamazepine, bisphenol A, clarithromycin, carbamazepine-10,11-epoxide, triclosan, diclofenac, PFOS, estrone, naproxen, ibuprofen, lidocaine, ketoprofen. The absence of a diffraction line (in XRD pattern) of iron oxide and zero modification found on the morphology of faujasite (in SEM images) were reported. This is due to the very low temperature used

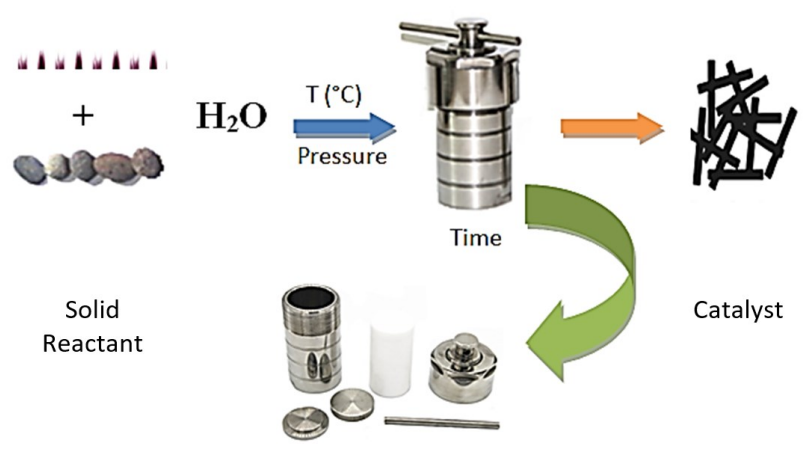

Figure 5. Schematic diagram of catalyst preparation method by hydrothermal synthesis. for the synthesis of catalyst [65]. Figure 4 (a) displays that Acid treatment is a comparatively better option for the enhancement of catalytic activity or activation of catalyst (coal fly ash). Wang et al. recommended that the $12 \mathrm{~h}$ of impregnation is necessary for the good catalyst [66]. Material like activated carbon can be used as a modifier. With the use of the modifier, it will increase the catalytic efficiency by increasing the pore volume. Figure 4 (b) shows the \% removal of p-NP using a modified catalyst. In the wet impregnation method for the preparation of appropriate catalyst, the effect of calcination and time have a significant role. For the making of a suitable catalyst, the calcinations temperature should be higher than $673 \mathrm{~K}$ and optimal time was measured as $12 \mathrm{hrs}$ [65]. Some authors used special equipment for the preparation of catalyst like rotary evaporator device. Impregnation of iron nitrate and nanocatalyst was employed in this device with certain temperature, pressure and time [67]. Table 1 shows brief literature survey of previous research work in which catalyst was prepared by WI method.

\subsection{Hydrothermal Method}

Hydrothermal synthesis occurs with the interaction between two solids under certain temperature and pressure. For the preparation of catalyst with the hydrothermal method, Teflon lined autoclave reactor made of stainless steel is required. This method is generally used for the making of zeolites and mixed oxides when molecular sieves come contact to each other. Hydrothermal method has opened a marvelous hope for the synthesis of various dimensional catalysts with an array of monodispersed pore size and redox properties [97]. Figure 5 depicts the procedure of the hydrothermal method for the synthesis of the catalyst. Bian et al. studied this method and prepared catalyst was used as a Surface-enhanced Raman scattering (SERS) substrate for the detection and decolorization of RG6 through photo-Fenton catalytic method. SERS is a susceptible, nondestructive and investigative instrument which can sense analytes even a solitary particle [98]. In this study, nanorod arrays $\left(\mathrm{CoFe}_{2} \mathrm{O}_{4} / \mathrm{Fe}_{2} \mathrm{O}_{3}\right)$ loaded with $\mathrm{Ag}$ was successfully fabricated on the carbon fiber cloth and that composite shows the bifunctional property (SERS) as well as a photo-Fenton catalyst [98]. Natural mineral (saprolite laterite ore) doped with multi-metal do not have the excellent photocatalytic activity, but it has good stability and recyclability performance. Most important 
Table 2. Catalyst prepared by HM method, optimum conditions and their respective performances.

\begin{tabular}{|c|c|c|c|c|c|c|c|c|c|}
\hline \multirow{2}{*}{$\begin{array}{l}\text { Pollutants } \\
\text { Treated }\end{array}$} & \multirow{2}{*}{$\begin{array}{l}\text { Catalyst used in hydro- } \\
\text { thermal method }\end{array}$} & \multicolumn{4}{|c|}{ Optimum Conditions } & \multicolumn{3}{|c|}{$\begin{array}{l}\text { Maximum } \\
\text { \% Degradation }\end{array}$} & \multirow[b]{2}{*}{ Ref. } \\
\hline & & $\begin{array}{l}\text { Initial } \\
\text { Conc. } \\
\text { (mg/L) } \\
\end{array}$ & $\mathrm{pH}$ & $\begin{array}{c}\text { Catalyst } \\
\text { dose } \\
(\mathrm{mg} / \mathrm{L}) \\
\end{array}$ & $\begin{array}{c}\mathrm{H}_{2} \mathrm{O}_{2} \\
(\mathrm{mM} / \mathrm{L})\end{array}$ & $\begin{array}{c}\mathrm{CO} \\
\mathrm{D} \\
(\%) \\
\end{array}$ & $\begin{array}{l}\text { Color } \\
(\%)\end{array}$ & $\begin{array}{c}\text { TOC } \\
(\%)\end{array}$ & \\
\hline $\begin{array}{l}\text { p- } \\
\text { Hydroxybenzoic } \\
\text { acid }\end{array}$ & $\mathrm{Fe} / \mathrm{Pd}-\mathrm{HNT}$ & $\begin{array}{c}10^{-3} \\
\mathrm{~mol} / \mathrm{L}\end{array}$ & 3.1 & $0.1 \mathrm{~g}$ & 0.12 & 52 & - & - & {$[112]$} \\
\hline $\begin{array}{l}\text { Rhodamine B } \\
\text { (RhB) }\end{array}$ & V-MCM-41 & $\begin{array}{c}0.02 \\
\mathrm{mmol} / \mathrm{L}\end{array}$ & 3 & $4 \mathrm{~g} / \mathrm{L}$ & 4 & - & 55 & - & [113] \\
\hline Rhodamine $6 \mathrm{G}$ & CuFeZSM-5 & $0.1 \mathrm{~g} / \mathrm{dm}$ & 3.4 & $0.15 \mathrm{~g}$ & 40 & & 100 & 51.8 & {$[50]$} \\
\hline Orange II & ZSM-5 & $\begin{array}{l}0.05 \\
\mathrm{~g} / \mathrm{dm}^{3}\end{array}$ & 3.5 & $0.15 \mathrm{~g}$ & 40 & 81.2 & 99.7 & - & {$[114]$} \\
\hline methylene blue & $\mathrm{Fe}_{5}\left(\mathrm{PO}_{4}\right)_{4}(\mathrm{OH})_{3} .2 \mathrm{H}_{2} \mathrm{O}$ & $100 \mathrm{~mL}$ & 4 & $0.5 \mathrm{~g} / \mathrm{L}$ & 9.8 & - & & - & [115] \\
\hline Phenol & $\mathrm{Fe}_{8} \mathrm{O}_{8}(\mathrm{OH})_{4.5}\left(\mathrm{SO}_{4}\right)_{1.75}$ & 100 & 5 & $1 \mathrm{~g} / \mathrm{L}$ & $\begin{array}{c}500 \\
\mathrm{mg} / \mathrm{L}\end{array}$ & - & - & 98 & {$[26]$} \\
\hline Methyl Orange & $\mathrm{TiO}_{2} / \mathrm{B}-\mathrm{FeOOH}$ & 100 & 4.5 & $0.2 \mathrm{~g} / \mathrm{L}$ & $0.3 \mathrm{~g} / \mathrm{L}$ & - & 41.2 & 90.09 & [116] \\
\hline RR195 & Fe-Cr-MIL-101 & $100 \mathrm{ppm}$ & 5.5 & 300 & $\begin{array}{c}136 \\
\mathrm{mg} / \mathrm{L}\end{array}$ & - & 98 & - & [117] \\
\hline dye pollution & $\begin{array}{l}\mathrm{HPB} \mathrm{Ba}_{2} \mathrm{Na}_{2}[\mathrm{HPWV} \\
4 \mathrm{WVI} \\
\left.8 \mathrm{O}_{40}\right] \cdot 26 \mathrm{H}_{2} \mathrm{O}(\mathrm{BaNaPW})\end{array}$ & $0.02 \mathrm{mM}$ & 2.5 & 14.6 & 6 & - & 95 & - & [99] \\
\hline Methylene blue & $\mathrm{Fe}_{2} \mathrm{O}_{3} @$ diatomite & 20 & 3 & $0.05 \mathrm{~g} / \mathrm{L}$ & 90 & - & 99 & - & {$[59]$} \\
\hline $\begin{array}{l}\text { methyl orange } \\
\text { sulfamethoxa- } \\
\text { zole }\end{array}$ & BFOs & 3 & 3 & & 16 & - & 99 & 99 & [118] \\
\hline phenol & (Fhy/MC) & 100 & 5 & $0.5 \mathrm{~g} / \mathrm{L}$ & 30 & 94.2 & - & - & [119] \\
\hline $\begin{array}{l}\text { organic pollu- } \\
\text { tants }\end{array}$ & $\begin{array}{l}\mathrm{CoS}_{2} / \mathrm{MWCNT} \\
\mathrm{CoS}_{2} \text { black }\end{array}$ & $\begin{array}{c}0.112 \\
\mathrm{mM}\end{array}$ & 3 & 1 & 30 & 60 & - & 43 & [120] \\
\hline Methyl Orange & $\mathrm{Fe}-\mathrm{Mn} / \mathrm{MCM}-41$ & 100 & $3.0 \pm 0.1$ & $1 \mathrm{~g} / \mathrm{L}$ & 5 & - & 100 & 60 & [121] \\
\hline $\begin{array}{l}\text { Dyeing } \\
\text { wastewater } \\
\text { treatment }\end{array}$ & $\mathrm{MBC}$ & 100 & 3 & $\begin{array}{l}0.1 \mathrm{~g} / 100 \\
\mathrm{~mL}\end{array}$ & $1 \mathrm{ml} / \mathrm{L}$ & $\begin{array}{l}47 \pm \\
3.3\end{array}$ & - & $\begin{array}{l}49 \pm \\
2.7\end{array}$ & [122] \\
\hline acid red G & {$\left[\mathrm{a}-\mathrm{Fe}_{2} \mathrm{O}_{3}\right]$} & 50 & 2 & $1 \mathrm{~g} / \mathrm{L}$ & 30 & 62 & 98 & - & [101] \\
\hline benzophenone-3 & CueMneO & 2 & 7 & 100 & 1 & 81.5 & - & - & [123] \\
\hline Bisphenol A & $\mathrm{Cu}$-doped $\mathrm{AlPO}_{4}$ & 25 & 7 & $1 \mathrm{~g} / \mathrm{L}$ & 10 & 92 & - & - & [124] \\
\hline DDT & $\mathrm{Ni} @ \mathrm{Fe}_{3} \mathrm{O}_{4}$ & $1 \mathrm{ppm}$ & 7 & $0.2 \mathrm{~g} / \mathrm{L}$ & 50 & 90 & - & - & [125] \\
\hline methylene blue & $\mathrm{MnO}_{2} @ \mathrm{SiO}_{2} \mathrm{NFM}$ & 10 & 6 & 10 & $15 \mathrm{~mL}$ & - & 95 & - & [126] \\
\hline
\end{tabular}


Table 2. ... (continued)

\begin{tabular}{|c|c|c|c|c|c|c|c|c|c|}
\hline \multirow{2}{*}{$\begin{array}{l}\text { Pollutants } \\
\text { Treated }\end{array}$} & \multirow{2}{*}{$\begin{array}{l}\text { Catalyst used in } \\
\text { hydrothermal } \\
\text { method }\end{array}$} & \multicolumn{4}{|c|}{ Optimum Conditions } & \multicolumn{3}{|c|}{$\begin{array}{c}\text { Maximum } \\
\% \text { Degradation }\end{array}$} & \multirow[b]{2}{*}{ Ref. } \\
\hline & & $\begin{array}{l}\text { Initial } \\
\text { Conc. } \\
(\mathrm{mg} / \mathrm{L})\end{array}$ & $\mathrm{pH}$ & $\begin{array}{l}\text { Catalyst } \\
\text { dose } \\
(\mathrm{mg} / \mathrm{L})\end{array}$ & $\begin{array}{c}\mathrm{H}_{2} \mathrm{O}_{2} \\
(\mathrm{mM} / \mathrm{L})\end{array}$ & $\begin{array}{l}\text { COD } \\
(\%)\end{array}$ & $\begin{array}{l}\text { Color } \\
(\%)\end{array}$ & $\begin{array}{c}\text { TOC } \\
(\%)\end{array}$ & \\
\hline methyl orange & MIL-100(Fe)/GO & 50 & 3 & $0.5 \mathrm{~g} / \mathrm{L}$ & 8 & - & 98 & 38 & [127] \\
\hline Reactive Black 5 & $\mathrm{rGO} / \mathrm{MnO}_{2}$ & $10 \mu \mathrm{m}$ & 5 & 20 & $6 \mathrm{~mL}$ & - & 95 & - & [128] \\
\hline Rhodamine B & $\begin{array}{l}\text { Amorphous Fe- } \\
\text { Zn- } \\
\text { oxide/hydrochar }\end{array}$ & 10 & 6.5 & $0.5 \mathrm{~g} / \mathrm{L}$ & 10 & - & 96.2 & - & [105] \\
\hline Bisphenol A & $\mathrm{Cu} / \mathrm{TUD}-1$ & $\begin{array}{l}100 \\
\text { ppm }\end{array}$ & 3.5 & $0.1 \mathrm{~g}$ & 90 & 90.4 & - & - & {$[45]$} \\
\hline Methyl Blue & $\mathrm{MnO}_{2}$ nanorods & $\begin{array}{l}0.16 \\
\mathrm{mM}\end{array}$ & 6.7 & $1 \mathrm{~g} / \mathrm{L}$ & 1.45 & - & 100 & - & [129] \\
\hline Methyl Orange & CuAl-LDH & 20 & 7 & 20 & $0.5 \mathrm{~mL}$ & - & 93.05 & - & [130] \\
\hline Methyl Blue & $\mathrm{Fe}_{3} \mathrm{O}_{4} / \mathrm{C} / \mathrm{Cu}$ & 100 & 6.9 & $0.5 \mathrm{~g} / \mathrm{L}$ & 163.7 & - & 97.2 & - & [131] \\
\hline PFOA & $\mathrm{Pb}-\mathrm{BFO} / \mathrm{rGO}$ & 50 & 5 & $1 \mathrm{~g} / \mathrm{L}$ & $44 \mathrm{mg} / \mathrm{L}$ & - & - & 90 & [132] \\
\hline $\mathrm{BPA}$ & $\mathrm{BiFeO}_{3}$ & 30 & 5 & $1 \mathrm{~g} / \mathrm{L}$ & $44 \mathrm{mg} / \mathrm{L}$ & - & - & 94 & [133] \\
\hline MB & $\mathrm{Fe}_{3} \mathrm{O}_{4} / \mathrm{SiO}_{2} / \mathrm{C}$ & 50 & 7.5 & 20 & $1 \mathrm{~mL}$ & 68.62 & 96 & - & [134] \\
\hline MO & $\mathrm{Fe}_{2} \mathrm{O}_{3} / \mathrm{MoS}_{2}$ & 20 & 7.5 & 10 & $0.4 \mathrm{~mL}$ & 56 & 99 & - & [135] \\
\hline MG \& RhB & $\mathrm{CuSe}$ & $20 \mathrm{~mL}$ & - & 10 & $1 \mathrm{~mL}$ & - & 96 & - & [136] \\
\hline $\begin{array}{l}\text { Refactory Pollu- } \\
\text { tants }\end{array}$ & DCAS Ns & 23 & 7 & $1 \mathrm{~g} / \mathrm{L}$ & 10 & - & 92.5 & 73.5 & [137] \\
\hline $\mathrm{C}_{6} \mathrm{H}_{5} \mathrm{OH}$ & $\mathrm{Cu}-\mathrm{SBA}-5$ & 100 & 8 & $1 \mathrm{~g} / \mathrm{L}$ & $\begin{array}{c}0.05 \\
\mathrm{~mol} / \mathrm{L}\end{array}$ & 100 & & 66.9 & [138] \\
\hline MB & MnSNTs & $50 \mathrm{ppm}$ & $2-3$ & 20 & $10 \mathrm{~mL}$ & - & 98.1 & - & [139] \\
\hline MB & $\begin{array}{l}\text { CNTs/ } \\
\text { Beta-FeOOH }\end{array}$ & 80 & 4.5 & $0.4 \mathrm{~g} / \mathrm{L}$ & $0.3 \mathrm{~g} / \mathrm{L}$ & - & 88.9 & - & [140] \\
\hline $\mathrm{RhB}$ & $\mathrm{Bi}_{25} \mathrm{FeO}_{40}$ & 80 & $4.2-4.4$ & $0.08 \mathrm{~g}$ & $1 \mathrm{~mL}$ & - & 98 & - & [141] \\
\hline $\mathrm{BPA}$ & $\begin{array}{l}\mathrm{d}-\mathrm{TiCuAl}-\mathrm{SiO}_{2} \\
\mathrm{Ns}\end{array}$ & 23 & 7 & $0.8 \mathrm{~g} / \mathrm{L}$ & 12 & - & - & $60-90$ & [142] \\
\hline MB & $\mathrm{Co}_{3} \mathrm{O}_{4}$ & 10 ppm & 7 & $1 \mathrm{mg}$ & $1 \mathrm{~mL}$ & - & 100 & - & [143] \\
\hline
\end{tabular}


Table 3. Catalyst prepared by SG method, optimum conditions and their achievements.

\begin{tabular}{|c|c|c|c|c|c|c|c|c|c|}
\hline \multirow{2}{*}{$\begin{array}{l}\text { Pollutants } \\
\text { Treated }\end{array}$} & \multirow{2}{*}{$\begin{array}{l}\text { Catalyst used in } \\
\text { sol-gel method }\end{array}$} & \multicolumn{4}{|c|}{ Optimum Conditions } & \multicolumn{3}{|c|}{$\begin{array}{c}\text { Maximum } \\
\text { \% Degradation }\end{array}$} & \multirow[b]{2}{*}{ Ref. } \\
\hline & & $\begin{array}{l}\text { Initial } \\
\text { Conc. } \\
\text { (ppm) }\end{array}$ & $\mathrm{pH}$ & $\begin{array}{c}\text { Catalyst } \\
\text { dose } \\
(\mathrm{ppm})\end{array}$ & $\begin{array}{l}\mathrm{H}_{2} \mathrm{O}_{2} \\
(\mathrm{mM})\end{array}$ & $\begin{array}{l}\text { COD } \\
(\%)\end{array}$ & $\begin{array}{l}\text { Color } \\
(\%)\end{array}$ & $\begin{array}{l}\text { TOC } \\
(\%)\end{array}$ & \\
\hline organic & Solid & 100 & 6.5 & 100 & $20 \mathrm{~mL}$ & 80 & - & - & [148] \\
\hline phenol & $\mathrm{Fe}_{2} \mathrm{O}_{3}-\mathrm{ZrO}_{2}$ & 100 & 7 & $0.8 \mathrm{~g} / \mathrm{L}$ & 112 & - & - & 56 & {$[18]$} \\
\hline bisphenol & $\begin{array}{l}\text { N-BFO nanoparti- } \\
\text { cles }\end{array}$ & 30 & 5.5 & $\begin{array}{c}0.25 \\
\mathrm{mmol} / \mathrm{L}\end{array}$ & 10 & 94 & - & - & [144] \\
\hline Rhodamine B & $\begin{array}{l}\text { Cu-embedded mes- } \\
\text { oporous alumina }\end{array}$ & $10 \mathrm{ppm}$ & 5.14 & $1 \mathrm{~g} / \mathrm{L}$ & $\begin{array}{l}1000 \\
\text { ppm }\end{array}$ & 98.53 & - & - & {$[21]$} \\
\hline Paracetamol & $\begin{array}{l}\text { five Fe-carbon } \\
\text { xerogels }\end{array}$ & 50 & $\begin{array}{l}6.2- \\
6.4\end{array}$ & 100 & 13.8 & 90 & - & 50 & {$[10]$} \\
\hline Tetracycline & $\mathrm{Fe}_{3} \mathrm{O}_{4} @$ void@ $\mathrm{TiO}_{2}$ & 40 & 3 & $0.25 \mathrm{~g} / \mathrm{L}$ & $0.377 \mathrm{M}$ & - & 75 & - & {$[12]$} \\
\hline $\begin{array}{l}\text { Sulfide and phe- } \\
\text { nolic } \\
\text { compounds }\end{array}$ & $\mathrm{CuO} / \mathrm{CeO}_{2}$ & 250 & 3.5 & $1 \mathrm{~g} / \mathrm{L}$ & $\begin{array}{l}1238 \\
\mathrm{mg} / \mathrm{L}\end{array}$ & 76 & - & - & [19] \\
\hline Antibiotics & $\begin{array}{l}\mathrm{Fe}_{2} \mathrm{O}_{3}, \mathrm{TiO}_{2} \text { and } \\
\mathrm{Fe}_{2} \mathrm{O}_{3}-\mathrm{TiO}_{2}\end{array}$ & 10 & 2.8 & 2 & 2.5 & 79 & - & 55 & {$[13]$} \\
\hline Phenol & $\mathrm{Fe}_{2} \mathrm{O}_{3} / \mathrm{SiO}_{2}$ & $1 \mathrm{~g} / \mathrm{L}$ & 2.7 & $5 \mathrm{~g}$ & $2.55 \mathrm{~g} / \mathrm{L}$ & 100 & - & 60 & [149] \\
\hline $\begin{array}{l}\text { Rhodamine B } \\
\text { dye }\end{array}$ & iron molybdate & 10 & - & $1 \mathrm{~g} / \mathrm{L}$ & 40 & - & 97 & - & {$[30]$} \\
\hline $\begin{array}{l}2- \\
\text { Chlorophenol }\end{array}$ & $\begin{array}{l}\text { metal-doped BFO } \\
\text { MNPsv }\end{array}$ & 50 & 5.5 & $0.04 \mathrm{~g}$ & 10 & 100 & - & 73 & [150] \\
\hline acid orange II & Fe-doped $\mathrm{TiO}_{2}$ & 20 & 4 & $0.5 \mathrm{~g} / \mathrm{L}$ & $\begin{array}{c}0.04 \\
\mathrm{~mol} / \mathrm{L}\end{array}$ & - & 99 & -- & [7] \\
\hline $\begin{array}{l}\text { Electrochemical } \\
\text { nitrate }\end{array}$ & $\begin{array}{l}\text { novel } \mathrm{Co}_{3} \mathrm{O}_{4} / \mathrm{Ti} \\
\text { cathode }\end{array}$ & 100 & 7 & $50 \mathrm{mg} / \mathrm{L}$ & $\begin{array}{c}0.05 \\
\mathrm{~mol} / \mathrm{L}\end{array}$ & $\begin{array}{l}\text { Nitro- } \\
\text { gen-85 }\end{array}$ & - & - & [151] \\
\hline methyl blue & Fe-GMCA & $\begin{array}{c}10 \\
\mathrm{mg} / \mathrm{mL}\end{array}$ & 3 & $0.02 \mathrm{~g}$ & & - & 99 & - & {$[20]$} \\
\hline $\begin{array}{l}\text { sulfamethoxa- } \\
\text { zole }\end{array}$ & $\begin{array}{l}\text { Magnetic carbon } \\
\text { xerogels }\end{array}$ & $50 \mu \mathrm{g} / \mathrm{L}$ & 3 & 80 & $500 \mathrm{mg} / \mathrm{L}$ & - & - & 42 & {$[152]$} \\
\hline toluene & $\begin{array}{l}\text { Ba-doping in } \\
\mathrm{BiFeO}_{3}\end{array}$ & 100 & 5.5 & 40 & $0.6-65$ & 85 & - & 94 & [153] \\
\hline methyl orange & $\mathrm{TiO}_{2} / \mathrm{Fe}_{3} \mathrm{O}_{4}$ & 100 & 6.5 & 100 & 0.4 & - & 88 & - & [148] \\
\hline Glycerol & $\mathrm{CuFe}_{2} \mathrm{O}_{4}$ & $\begin{array}{l}68.4 \\
\mathrm{mM}\end{array}$ & $\begin{array}{l}0.1 \\
\mathrm{~g} / \mathrm{L}\end{array}$ & $5 \mathrm{~g} / \mathrm{L}$ & 819.5 & $\begin{array}{c}\text { Degra- } \\
\text { dation- } \\
40\end{array}$ & - & - & [154] \\
\hline 2-chlorophenol & $\begin{array}{l}\text { mesostructured } \\
\text { silica nanoparticles }\end{array}$ & 50 & 5 & $0.4 \mathrm{~g} / \mathrm{L}$ & 0.156 & 99.9 & - & - & [96] \\
\hline Orange I & $\mathrm{Fe}-\mathrm{B}$ & $0.2 \mathrm{mM}$ & 3 & $0.5 \mathrm{~g} / \mathrm{L}$ & 10 & - & - & 75 & {$[155]$} \\
\hline
\end{tabular}


part of this study is that the leaching solution can be taken as a precursor solution for the synthesis of catalyst [58]. Similarly, HPB-3D inorganic heteropoly blue [99], $\mathrm{MnO}_{2}$-templated iron oxide-coated diatomite [59], magnetic g$\mathrm{C}_{3} \mathrm{~N}_{4} / \mathrm{a}-\mathrm{Fe}_{2} \mathrm{O}_{3} / \mathrm{Fe}_{3} \mathrm{O}_{4}$ composite [100], burgerlike $\mathrm{a}-\mathrm{Fe}_{2} \mathrm{O}_{3}$ catalyst [101], 3D hierarchical nanostructured hematite $\left(\mathrm{Fe}_{2} \mathrm{O}_{3}\right)$ [102], $\mathrm{MoS}_{2} / \mathrm{Fe}_{3} \mathrm{O}_{4}$ nano composite [56], Mixed a$\mathrm{Fe}_{2} \mathrm{O}_{3} / \mathrm{Bi}_{2} \mathrm{WO}_{6}$ oxides [103], $\mathrm{Fe}_{2} \mathrm{O}_{3} /$ expanded perlite $\left(\mathrm{Fe}_{2} \mathrm{O}_{3}\right.$-Ep) composite catalyst [104], FeZn-oxide/hydrochar [105], $\mathrm{Ag}_{3} \mathrm{PO}_{4} / \mathrm{CuO}$ composites [106], zinc oxide decorated iron oxide/reduced graphene oxide nanocomposite [107], Cu-doped $\mathrm{LaFeO}_{3}$ [108], $\mathrm{Fe}$ (III)tartrate $/ \mathrm{TiO}_{2}$ nano tubular [109], $\mathrm{Fe}_{3} \mathrm{O}_{4}$-GO nano composite [27], $\mathrm{ZnFe}_{2} \mathrm{O}_{4}$ nanostructures [110], $\mathrm{BiFeO}_{3}-\mathrm{g}-\mathrm{C}_{3} \mathrm{~N}_{4}$ compound [55] were prepared by the hydrothermal method and used as photocatalysts. Recently, some researchers investigated that the metal doped $\mathrm{Fe}_{2} \mathrm{O}_{3}$ catalyst has good stability and potential to enhance the photo degradation [111]. Table 2 shows the brief literature survey of previous research work in which catalyst was prepared by hydrothermal method (HM) method.

\subsection{Sol-gel Method}

Sol-gel is the second largest method used for the preparation of xerogels or aerogels. Sol is a colloidal suspension of solid particles in a solution with the particle range from $\mathrm{nm}$ to $\mu \mathrm{m}$ size. Gel is defined as the solid surrounded by liquid. In the first step for the synthesis of catalyst monomer, metallic oxide reacts with water and produce a polymeric gel which is considered as an oxide gel. Then in the second step, condensation occur which forms gel by dehydration and create a Metal-O-Metal bond. The third step is a formation of a gel by drying or aging which produces a cross-linked gel. During the progress of gel formation, viscosity also increases till the sol-gel transition point. Then with further increase in viscosity in cross-

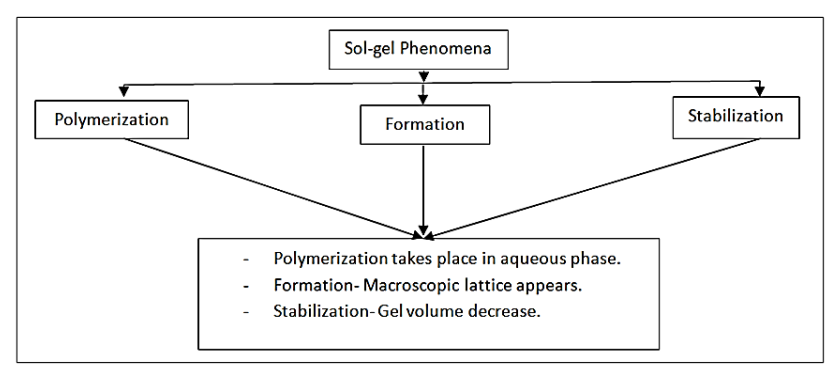

Figure 6. The basic phenomena of the sol-gel process. linking results in maximum density which occurs by aging and drying. The role of $\mathrm{pH}$ is very important in the synthesis process because lower value of the same parameter gives a lower cross-linking substance. Similarly, aging, temperature, water alkoxide ratio, alkyl group (length, molecular weight) and oxidation state are also important parameters. One of the parameters is gel structure which completely depends on hydroxyl group present in the gel after drying. Figure 6 displays the fundamental phenomena of the sol-gel process.

Alhmoud et al. investigated that mixed oxide containing iron has great efficacy to degrade phenol and their derivatives $[2,18,19,144,145]$. The author used different metals and synthesized catalyst by sol-gel method for example: $\mathrm{CuFe}_{1.2} \mathrm{O}_{2.8}, \mathrm{BaFe}_{7.2} \mathrm{O}_{11.8}$, $\mathrm{BaFe}{ }_{7.2} \mathrm{Cu}_{2} \mathrm{O}_{13.8}, \quad \mathrm{BaFe}_{5.4} \mathrm{~V}_{3} \mathrm{O}_{16.6}$, $\mathrm{BaFe}_{4.8} \mathrm{Cu}_{2} \mathrm{~V}_{3} \mathrm{O}_{17.7}$, and $\mathrm{Ag}_{2} \mathrm{Fe}_{5.4} \mathrm{~V}_{3} \mathrm{O}_{16.6}$. In addition, it was also found that catalytic activity was suppressed with the increase of temperature [17]. Another researcher developed a catalyst $\mathrm{rGO}-\mathrm{Fe}_{3} \mathrm{O}_{4}-\mathrm{TiO}_{2}$ with a ratio of $1: 1: 2$ and about $99 \%$ of dye was degraded in only 6 minutes [4]. Similarly, many scientists worked with the prepared catalyst by sol-gel method for degradation of dye like Rhodamine B and $\mathrm{Cu}$-Embedded Alumina [21], acid orange-II and Fe-doped $\mathrm{TiO}_{2}$ [7], methylene blue dye and Ag$\mathrm{SiO}_{2} @-\mathrm{Fe}_{2} \mathrm{O}_{3}$ [146], Orange $\mathrm{G}$ and $\mathrm{TiO}_{2} /$ stainless steel mesh photo-electrode [147], methyl orange and $\mathrm{TiO}_{2} / \mathrm{Fe}_{3} \mathrm{O}_{4}$ [148]. Table 3 shows the brief literature survey of previous

Table 4. Precipitation parameters and their effects on seeding [2].

\begin{tabular}{ll}
\hline Parameters & Effects on crystal \\
\hline $\mathrm{pH}$ & Phase \\
Solution composition & $\begin{array}{l}\text { Phase, purity, precipitation } \\
\text { composition } \\
\text { Purity, crystallinity, textur- } \\
\text { al properties }\end{array}$ \\
Aging & Textural properties \\
Additives & Phase, textural properties \\
Temperature & $\begin{array}{l}\text { Precipitate composition, } \\
\text { homogeneity }\end{array}$ \\
Mixing sequence & $\begin{array}{l}\text { Textural properties, crystal- } \\
\text { linity } \\
\text { Sarticle size, rate of precipi- } \\
\text { tation }\end{array}$ \\
Super saturation & $\begin{array}{l}\text { Phase, homogeneity } \\
\text { Morphology, textural prop- } \\
\text { Precipitating agent }\end{array}$ \\
Anion & \begin{tabular}{l} 
erties \\
\hline
\end{tabular}
\end{tabular}


Table 5. Catalyst prepared by (PPT and CO-PPT) method, optimum condition and their achievements.

\begin{tabular}{|c|c|c|c|c|c|c|c|c|c|}
\hline \multirow[b]{2}{*}{ Pollutant Treated } & \multirow{2}{*}{$\begin{array}{l}\text { Catalyst used in } \\
\text { precipitation and } \\
\text { co-precipitation } \\
\text { method }\end{array}$} & \multicolumn{4}{|c|}{ Optimum Conditions } & \multicolumn{3}{|c|}{$\begin{array}{c}\text { Maximum } \\
\text { \% Degradation }\end{array}$} & \multirow[b]{2}{*}{ Ref. } \\
\hline & & $\begin{array}{l}\text { Initial } \\
\text { Conc. } \\
\text { (ppm) }\end{array}$ & $\mathrm{pH}$ & $\begin{array}{l}\text { Catalyst } \\
\text { dose } \\
(\mathrm{ppm})\end{array}$ & $\begin{array}{l}\mathrm{H}_{2} \mathrm{O}_{2} \\
(\mathrm{mM})\end{array}$ & $\begin{array}{l}\text { COD } \\
(\%)\end{array}$ & Color (\%) & $\begin{array}{l}\text { TOC } \\
(\%)\end{array}$ & \\
\hline $\mathrm{NiZn}$ & $\overline{\mathrm{Fe} / \mathrm{L}}$ & $1 \mathrm{mM}$ & 7 & 174 & 10 & 67 & - & 100 & [169] \\
\hline Thallium & $\mathrm{FeSO}_{4} \cdot 7 \mathrm{H}_{2} \mathrm{O}$ & $500 \mu \mathrm{m}$ & 2.5 & 21.58 & 53.96 & 96.54 & - & 70.42 & [170] \\
\hline Methyl Orange & $\mathrm{Fe}_{3} \mathrm{O}_{4} / \mathrm{MWCNTS}$ & 50 & 2 & $2 \mathrm{~g} / \mathrm{L}$ & 19.38 & - & - & - & [159] \\
\hline Methylene Blue & $\mathrm{MFe}_{2} \mathrm{O}_{4}$ & 10 & - & 100 & $4 \mathrm{~mL}$ & - & 99.5 & - & [157] \\
\hline Acid orange 7 & $\mathrm{~K}-\mathrm{FeOx}-2 / 2-3 \mathrm{H}$ & 25 & 3 & $3 \mathrm{~g} / \mathrm{L}$ & 5 & -- & 80 & - & [171] \\
\hline Organic Pollutant & $\mathrm{Fe}_{2} \mathrm{GeS}_{4}$ & 20 & 7 & $0.3 \mathrm{~g} / \mathrm{L}$ & 50 & 74.2 & $\begin{array}{l}\mathrm{MO}=66.2 \\
\mathrm{MB}=56.3\end{array}$ & - & [172] \\
\hline 4-Bromophenol & $\begin{array}{l}\text { Se-doped } \mathrm{CuO} \\
\text { NPs }\end{array}$ & 0.2 & - & 10 & $0.038 \mathrm{M}$ & 99.5 & - & - & [63] \\
\hline Humic acids (HAs) & $\begin{array}{l}\text { ferrioxalate com- } \\
\text { plexes }\end{array}$ & 100 & 2.8 & 40 & 42 & HA 95 & - & - & [173] \\
\hline $\begin{array}{l}\text { Chlordimeform } \\
\text { insecticide }\end{array}$ & $\mathrm{Fe}_{3} \mathrm{O}_{4}-\mathrm{Cs}$ & 37.5 & 3 & 0.5 & $0.104 \pm 0.001$ & - & - & 80 & [62] \\
\hline $\begin{array}{l}\text { Methyl Orange } \\
\text { Sulfamethoxazole }\end{array}$ & $\mathrm{BFO} / \mathrm{H}_{2} \mathrm{O}_{2} / \mathrm{PMS}$ & $3 \mathrm{mg} / \mathrm{L}$ & 3 & $0.12 \mathrm{~g} / \mathrm{L}$ & 40 & - & 99 & 99 & [118] \\
\hline Sulfate & SBR & $200 \mathrm{~mL}$ & 2 & $8 \mathrm{mM} / \mathrm{L}$ & 40 & 77.9 & - & - & [174] \\
\hline $\begin{array}{l}\text { Benzophenone-3 } \\
\text { (BP-3) }\end{array}$ & CueMneO & 2 & 7 & 100 & 40 & 81.5 & - & - & [123] \\
\hline Phenol & $\mathrm{NiFe}_{2} \mathrm{O}_{4}$ & 250 & 3 & $2 \mathrm{~g} / \mathrm{L}$ & 120 & $95 \pm 3.4$ & - & - & [175] \\
\hline $\mathrm{RhB}$ & $\mathrm{Fe}_{3} \mathrm{O}_{4} / \mathrm{Al}-\mathrm{B}$ & 40 & 3 & $1 \mathrm{~g} / \mathrm{L}$ & 50 & RhB 99.9 & - & - & [168] \\
\hline $\begin{array}{l}\text { Phenol } \\
\text { p-Nitrophenol }\end{array}$ & $\mathrm{Fe}_{3} \mathrm{O}_{4} / \mathrm{MWCNT}$ & 100 & 3 & 1 & 10 & 99.20 & - & - & [167] \\
\hline SDBS & ZVI & 60 & 6 & $2 \mathrm{~g} / \mathrm{L}$ & - & - & - & 77.8 & [176] \\
\hline $\begin{array}{l}\text { Alkalinity } \\
\text { Ammonium nitro- } \\
\text { gen }\end{array}$ & $\mathrm{Fe}^{2+}$ & 100 & 2.8 & 60 & 120 & 58 & $\begin{aligned} \mathrm{DOC} & =60 \\
\mathrm{TSS} & =88\end{aligned}$ & - & [177] \\
\hline Congo red & $\begin{array}{l}\text { Cobalt-copper } \\
\text { oxalate }\end{array}$ & 100 & 7 & 100 & $100 \mathrm{mg} / \mathrm{L}$ & - & 100 & - & [166] \\
\hline levofloxacin & $\begin{array}{l}\text { ferrioxalate com- } \\
\text { plexes }\end{array}$ & 20 & 5 & 2 & $20 \mathrm{mg} / \mathrm{L}$ & $\begin{array}{l}\mathrm{DOC}=86 \\
\mathrm{LEV}=71\end{array}$ & - & - & [178] \\
\hline Methyl orange & $\mathrm{NiFe}\left(\mathrm{C}_{2} \mathrm{O}_{4}\right)_{\mathrm{x}}$ & 20 & $\begin{array}{l}3- \\
10\end{array}$ & $0.4 \mathrm{~g} / \mathrm{L}$ & 10 & - & 98 & - & [165] \\
\hline catechol & $\mathrm{Fe}_{3} \mathrm{O}_{4}-\mathrm{CeO}_{2}$ & $10 \mathrm{mM}$ & 2.4 & 50 & 30 & 89.2 & - & - & [164] \\
\hline Reactive Black 5 & $\begin{array}{l}\text { modified } \\
\text { cigarette filter } \\
(\mathrm{MCF})\end{array}$ & 50 & 3 & $1 \mathrm{~g} / \mathrm{L}$ & $100 \mathrm{mg} / \mathrm{L}$ & - & 99.09 & - & [179] \\
\hline Enrofloxacin & $\mathrm{Fe}^{2+}$ & 100 & $2-3$ & 28.3 & $738 \mathrm{mg} / \mathrm{L}$ & - & - & $>58$ & [180] \\
\hline
\end{tabular}


Table 5. ... (continued).

\begin{tabular}{|c|c|c|c|c|c|c|c|c|c|}
\hline \multirow[b]{2}{*}{ Pollutant Treated } & \multirow{2}{*}{$\begin{array}{l}\text { Catalyst used in } \\
\text { precipitation } \\
\text { and co- } \\
\text { precipitation } \\
\text { method }\end{array}$} & \multicolumn{4}{|c|}{ Optimum Conditions } & \multicolumn{3}{|c|}{$\begin{array}{c}\text { Maximum } \\
\text { \% Degradation }\end{array}$} & \multirow[b]{2}{*}{ Ref. } \\
\hline & & $\begin{array}{l}\text { Initial } \\
\text { Conc. } \\
\text { (ppm) }\end{array}$ & $\mathrm{pH}$ & $\begin{array}{l}\text { Catalyst } \\
\text { dose } \\
(\mathrm{ppm})\end{array}$ & $\begin{array}{l}\mathrm{H}_{2} \mathrm{O}_{2} \\
(\mathrm{mM})\end{array}$ & $\begin{array}{c}\text { COD } \\
(\%)\end{array}$ & $\begin{array}{c}\text { Color } \\
(\%)\end{array}$ & $\begin{array}{c}\text { TOC } \\
(\%)\end{array}$ & \\
\hline $\begin{array}{l}\text { Methylene blue } \\
\text { Rhodamine B }\end{array}$ & La-Fe MMT & 100 & $\begin{array}{l}\text { Neu } \\
\text { tral }\end{array}$ & $0.1 \mathrm{~g} / \mathrm{L}$ & 30 & 75 & $\begin{array}{l}\mathrm{MB}=97 \\
\mathrm{RhB}=96\end{array}$ & - & {$[163]$} \\
\hline Dye & $\begin{array}{l}\text { diato- } \\
\text { mite/ } / \mathrm{Fe}_{2} \mathrm{O}_{3} / \mathrm{TiO}_{2}\end{array}$ & $2.9 \times 10^{-5}$ & 7 & $2 \mathrm{~g} / \mathrm{L}$ & $210 \mu \mathrm{L}$ & - & 96 & - & {$[162]$} \\
\hline nitrobenzene & Co-Fe LDH & $20 \mu \mathrm{L}$ & 2.7 & $1 \mathrm{~g} / \mathrm{L}$ & $\begin{array}{c}500 \\
\mathrm{mg} / \mathrm{L}\end{array}$ & $\begin{array}{l}\text { Nitro- } \\
\text { benzene } \\
100\end{array}$ & - & 75 & {$[161]$} \\
\hline Reactive Yellow 15 & $\mathrm{Fe} / \mathrm{CuO}$ & & 2 & $4 \mathrm{~g} / \mathrm{L}$ & 50 & - & 98 & - & {$[160]$} \\
\hline Micropollutants & $\mathrm{Fe}^{2+}$ & 100 & 3.72 & 1,755 & $\begin{array}{c}26,422 \\
\mathrm{mg} / \mathrm{L}\end{array}$ & 54 & - & $\begin{array}{c}\text { Mi- } \\
\text { cropollu } \\
\text { tants=9 } \\
0-99 \%\end{array}$ & {$[181]$} \\
\hline $\begin{array}{l}\text { p-arsanilic acid (p- } \\
\text { ASA) }\end{array}$ & $\mathrm{Fe}^{2+}$ & 10 & 3 & $\begin{array}{c}0.53 \\
\mathrm{mM} / \mathrm{L}\end{array}$ & 2.12 & $\begin{array}{c}\mathrm{p}- \\
\mathrm{ASA}=100 \\
\%\end{array}$ & - & - & {$[182]$} \\
\hline Phenol & $\mathrm{Fe}_{3} \mathrm{O}_{4}-\mathrm{GO}$ & 20 & 5 & $0.25 \mathrm{~g} / \mathrm{L}$ & 10 & 98.8 & - & 81.3 & {$[27]$} \\
\hline Methyl Orange & $\mathrm{Fe}_{3} \mathrm{O}_{4} @ \mathrm{HG}$ & 10 & 3.5 & 3 & $1.2 \mathrm{~mL}$ & - & 87.68 & - & [183] \\
\hline Methylene Blue & $\mathrm{BaFe}_{12} \mathrm{O}_{19}$ & 10 & - & $0.75 \mathrm{~g} / \mathrm{L}$ & 12 & - & 70.8 & $\begin{array}{c}\text { Mineral- } \\
\text { ization= } \\
63.7 \%\end{array}$ & {$[184]$} \\
\hline $\begin{array}{l}\text { reactive brilliant } \\
\text { blue }\end{array}$ & Fe-Mn-sepiolite & 50 & 2.5 & $0.4 \mathrm{~g}$ & $3 \mathrm{~mL}$ & - & - & 91.98 & {$[185]$} \\
\hline glycerin & $\begin{array}{l}\mathrm{Fe}^{2+}-\mathrm{HA} \\
\mathrm{Fe}^{2+}-\mathrm{BQ}\end{array}$ & 100 & 3 & $5 \mathrm{mM} / \mathrm{L}$ & 50 & - & - & 90 & {$[186]$} \\
\hline $\begin{array}{l}\text { beverage industrial } \\
\text { effluent }\end{array}$ & $\mathrm{Fe}(\mathrm{II}) / \mathrm{PS}$ & 500 & 2.9 & 375 & $\begin{array}{l}4000 \\
\mathrm{mg} / \mathrm{L}\end{array}$ & $\begin{array}{c}\text { Mineral }= \\
76\end{array}$ & - & 93 & {$[187]$} \\
\hline Orange II & $\mathrm{Fe}_{3} \mathrm{O}_{4} / \mathrm{Al}-\mathrm{Fe}-\mathrm{P}-\mathrm{B}$ & $\begin{array}{c}0.23 \\
\mathrm{mM} / \mathrm{L}\end{array}$ & 4 & $1 \mathrm{~g} / \mathrm{L}$ & 1 & 92.2 & 99.3 & - & {$[188]$} \\
\hline $\begin{array}{l}2,4,5- \\
\text { trichlorophenoxya- } \\
\text { cetic acid }\end{array}$ & $\mathrm{cLDHs}$ & 100 & 3.8 & $4 \mathrm{~g} / \mathrm{L}$ & 2.73 & - & - & $80-95$ & {$[189]$} \\
\hline $\begin{array}{l}\text { cibacron brilliant } \\
\text { red 3B-A (BR3B-A) }\end{array}$ & $\mathrm{Fe}_{78} \mathrm{Si}_{9} \mathrm{~B}_{13}$ & $0.02 \mathrm{mM}$ & 2.5 & $14.6 \mathrm{mg}$ & 6 & - & 100 & - & [99] \\
\hline $\mathrm{BPA}$ & $\mathrm{Fe}_{3} \mathrm{O}_{4}-\mathrm{TA}$ & $0.5 \mu \mathrm{M}$ & 6.6 & $1 \mathrm{~g} / \mathrm{L}$ & 10 & $\begin{array}{c}\mathrm{BPA}=80 \\
\%\end{array}$ & - & - & {$[190]$} \\
\hline Sludge & $\mathrm{MFeNp}$ & $100 \mathrm{~mL}$ & 3 & $50 \mathrm{mg} / \mathrm{g}$ & $\begin{array}{c}500 \\
\mathrm{mg} / \mathrm{g}\end{array}$ & $\begin{array}{l}\text { Sludge= } \\
85.9 \%\end{array}$ & - & - & [191] \\
\hline
\end{tabular}


research work in which catalyst was prepared by sol-gel (SG) method.

\subsection{Precipitation and Co-precipitation Method}

Two basic things of precipitation are nucleation and growth. When high supersaturation is achieved then nucleation comes into the picture (formation of solid crystal) and growth means approaching an equilibrium state with the new phase. Formed crystal could be homogeneous or heterogeneous. Homogeneous nucleation occurs when molecules interact with each other with the same phase under supersaturation condition and irreversible crystal is formed while in heterogeneous nucleation. For the successful precipitation, some necessary parameters should be controlled like initial $\mathrm{pH}, \mathrm{pH}$ variation during the process, the rate of addition of solution, order, types of mixing, maturation etc. Due to its simple nature and cost-effectiveness, this method is the most used all over the world. Few disadvantages are product separation after precipitation, higher salt amount present in solution, etc. [97].

In the co-precipitation method, two different ions (cation and anion) are associated with a fixed composition. If the same ions interact, then characteristic (solubility constant and supersaturation value) and properties of formed seed will also change with time. For the preparation of mixed oxide, solubility is a very important factor in the case of co-precipitation. Similar to precipitation, in the co-precipitation also $\mathrm{pH}$ has a great role. There are two methods for seeding by the co-precipitation method: a) constant $\mathrm{pH}$ method, and b) variable $\mathrm{pH}$ method during the process. Stirring can also change the property of crystal in the precipitation. Parameters and their effects on crystal are listed in Table 4 [97].

Vinosha et al. investigated the effect of different $\mathrm{pH}(9-12)$ on crystal formation in terms of structure, optical and magnetic properties of $\mathrm{CoFe}_{2} \mathrm{O}_{4}$ nanoparticle which was prepared by co-precipitation method. Authors also investigated that the prepared catalyst will be much effective in the Fenton process using methylene blue as a model pollutant [61]. Selenium has a photo absorption property and the great efficiency to improve the in-situ generation of hydroxyl radical. Sharma \& Dutta synthesized the catalyst using selenium and studied their optical, morphological and structural properties of Se-doped $\mathrm{CuO}$ NPs nanoparticles [63]. Gan et al. prepared a catalyst by oxidation, reduction and co-precipitation, and that catalyst exhibits great catalytic ability and efficient reusability performance [94]. The researchers studied the application of prepared catalyst by precipitation and co-precipitation method in the various field like color removal and recalcitrant organic compound degradation. Some examples of dye as model pollutant, and prepared catalyst are $\mathrm{Ni}_{1-\mathrm{x}} \mathrm{Zn}_{\mathrm{x}} \mathrm{Fe}_{2} \mathrm{O}_{4}$ and methylene blue [156-158], methyl orange and $\mathrm{Fe}_{3} \mathrm{O}_{4} / \mathrm{MWCNTs}$ nanocomposites [159], Reactive Yellow 15 and $\mathrm{Fe} / \mathrm{CuO}$ catalyst [160], nitrobenzene and Co-Fe layered double hydroxide (Co-Fe LDH) [161], methylene blue and Magnetic diatomite (Kieselguhr) $/ \mathrm{Fe}_{2} \mathrm{O}_{3} / \mathrm{TiO}_{2}$ composite [162], RhB and methylene blue (MB)

Table 6. The possible structure of matter and their properties.

\begin{tabular}{|c|c|c|}
\hline Shape & Figure & Properties \\
\hline Sphere & & $\begin{array}{l}\text { Low manufacturing costs } \\
\text { The relatively high-pressure drop } \\
\text { Large diffusion length HDS, Methanation }\end{array}$ \\
\hline Granules & & $\begin{array}{l}\text { Not common, Low-surface-area catalysts Ammonia } \\
\text { Synthesis, Formaldehyde }\end{array}$ \\
\hline Pellets & & $\begin{array}{l}\text { Regular shape; Most common } \\
\text { Good strength. CO shift Hydrogenation }\end{array}$ \\
\hline Extrudates cylinder & & Low-pressure drop; Poor strength HDS \\
\hline Ring & & $\begin{array}{l}\text { High strength; Low-pressure drop } \\
\text { Small diffusion length. Steam Reforming }\end{array}$ \\
\hline Monoliths ceramics & & $\begin{array}{l}\text { Low pressure drop; Insensitive to dust; Small diffusion } \\
\text { length Exhaust gas cleaning }\end{array}$ \\
\hline
\end{tabular}


and La-Fe montmorillonite (La-Fe MMT) [163], orange $\mathrm{G}$ and $\mathrm{Fe}_{3} \mathrm{O}_{4} / \mathrm{CeO}_{2}$ composite [94], catechol and $\mathrm{Fe}_{3} \mathrm{O}_{4}-\mathrm{CeO}_{2}$ metal oxide [164], methyl orange and $\mathrm{Ni}-\mathrm{Fe}\left(\mathrm{C}_{2} \mathrm{O}_{4}\right)_{\times}$[165], Rhodamine $\mathrm{B}$ dye and Iron molybdate $\mathrm{Fe}_{2}\left(\mathrm{MoO}_{4}\right)_{3}$ nanopowders [30], congo red and cobalt-copper oxalate and cobalt oxalate nanofibers [166], Phenol and $\mathrm{Fe}_{3} \mathrm{O}_{4} / \mathrm{MWCNT}$ [167], rhodamine B $(\mathrm{RhB})$ and $\mathrm{Al}$ pillared bentonite- $\mathrm{Fe}_{3} \mathrm{O}_{4}$ nanocomposites $\left(\mathrm{Fe}_{3} \mathrm{O}_{4} / \mathrm{Al}-\mathrm{B}\right)$ [168]. Table 5 shows the brief literature survey of previous research work in which catalyst was prepared by precipitation and co-precipitation (PPT and CO-PPT) method.

\section{Modification and Application of Modi- fied Catalyst in the Fenton Process}

Modified Fenton's catalyst (MFC) is directly related with the Fenton's chemistry. British chemist Henry J.H. Fenton first established the use of Fenton's chemistry (1894), through the degradation of tartaric acid by the use of iron, hydrogen peroxide under acidic conditions. MFC process was developed to overcome the application of Fenton's reagent in its conventional form (such as: acidic $\mathrm{pH}$, iron leaching, sludge formation, reusability, etc.). The main aim of modification of Fenton's catalyst is to stop iron leaching and chelating. To prevent $\mathrm{pH}$ fluctuation during process, modification of Fenton's catalyst can be done at normal $\mathrm{pH}$ of 5-7. Chelated catalyst has a better attraction towards the iron compared with other metals, hence the iron loss is negligible. The fundamental Fenton reaction is between $\mathrm{Fe}^{2+}$ and hydrogen peroxide and produces hydroxyl radical $(\cdot \mathrm{OH}), \mathrm{Fe}^{3+}$, and $\mathrm{OH}$. Modified Fenton's pro-

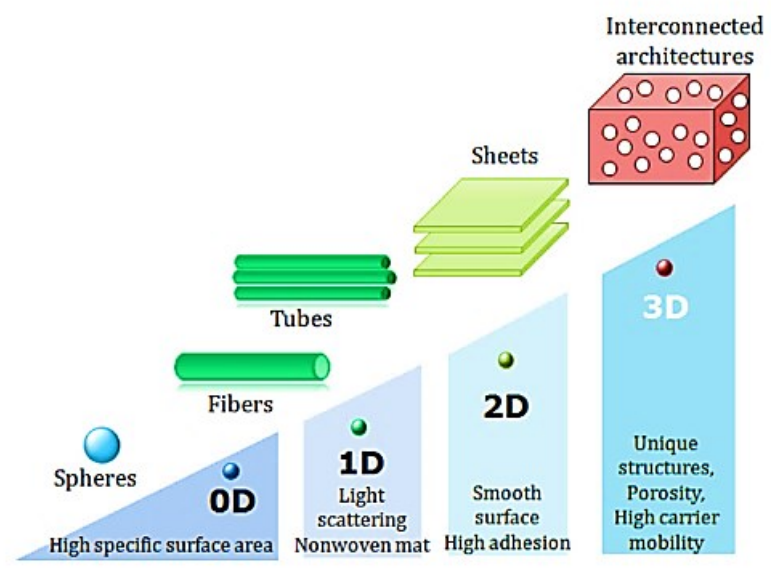

Figure 7. Various dimension and their expected properties [203] (Reprinted with permission from Ref. [203] copyright from Elsevier). cesses produce superoxide radical anion $\left(\mathrm{O}_{2} \cdot{ }^{\cdot}\right)$ or hydroperoxyl ion $\left(\mathrm{HO}_{2}\right.$ ) which endorses the chemical reaction. Similar to hydroxyl radical, superoxide and hydroperoxide anions are also responsible for the oxidation and adsorption [192].

To enhance the catalytic efficiency, many researchers have been working on the modification of catalyst. Reyes et al. used two different ligand-based iron complex and compared their efficiency with the Fenton process. They found that modified Fenton's reagent is more efficient (at neutral $\mathrm{pH}$ ) than simple Fenton reaction (at low pH) [193]. Textile effluent contains dye, surfactants, additives, and hazardous matter. Darshna \& Yogesh used fly ash along with iron dust instead of iron salt for the degradation of color and chemical oxygen demand (COD) and got significant results [194]. Jennifer et al. employed a novel technique, rotating disk-slurry electrode (iron salt on the electrode and activated carbon) for the preparation of catalyst and examined their performance [195]. Mansoorian et al. studied both the process (Fenton and modified Fenton) for the dye decolorization with the same operating parameters and observed that modified Fenton's reagent is more efficient [196]. Similarly, Ganesan et al. (decolorization of textile dyeing) [197], Lewis et al. (degradation of trichloroethylene) [198], Wang et al. (degradation of pnitrophenol) [66], Lee et al. (Degradation of phenol) [199], Barbusinski et al. (decolorization of dye wastewater) [194], Zhao et al. (removal of textile dyes) [200], Pradisty et al. (degradation of phenol) [201], Tony et al., (mineralization of an oil-water emulsion) [202], Sadek et al. (the treatment of effluents from paint industry) investigated on modified Fenton's reagent [67].

\subsection{Physical Modification}

The physical modification is directly concerned with the study of morphology. The term morphology is related to the shape, structure, and form of material. The main aim of modification of the catalyst is to enhance their catalytic activity. The shape of any matter could be zero-dimensional, 1 -dimensional, 2-dimensional, or multidimensional. Figure 7 shows the various dimension and their expected properties. Similarly, the matter could be of various type shapes like a sphere, pellet, ring, monolith, wagon wheel, monolith, extruded cylindrical, gauzes, etc. Table 6 shows the various shape and their properties. 
Lee et al. reviewed especially on the modification of $\mathrm{TiO}_{2}$ including metal, carbon or noncarbon material incorporated. Photocatalytic activity can be enhanced by the optimization of structure and size of the matter. The shape of $\mathrm{TiO}_{2}$ catalyst with nanoscale can increase the adsorption capacity which is very helpful to the wastewater treatment, particular in Fenton's process. In addition, photocatalytic performance gets affected on the surface of $\mathrm{TiO}_{2}$ dominant behavior of the $\mathrm{e} / \mathrm{h}^{+}$. Compared to the unmodified catalyst, modified catalyst showed the diversity of photocatalyst characteristics and significant catalytic activity performance. Similarly, compared to particle form, nanotube structure $\mathrm{TiO}_{2}$ exhibits great potential to degrade recalcitrant organic compound and mineralize them into the non-toxic compounds. Nano-sized materials have the high specific area, ability to charge separation on the catalyst surface, enhanced oxidative property along with opacity, but directly this cannot be applied in wastewater treatment due to the aggregation of nano-particle [203]. Apart from the titanium, ferrite spinel has also excellent potential in the field of advanced oxidation technology. Ferrite spinel consists mostly of divalent ion which is distributed in the cubic close packing of tetrahedral or octahedral coordination sites. Catalytic performance is directly related to the oxidation and reduction property and distribution of metal ion on the configuration [204]. In
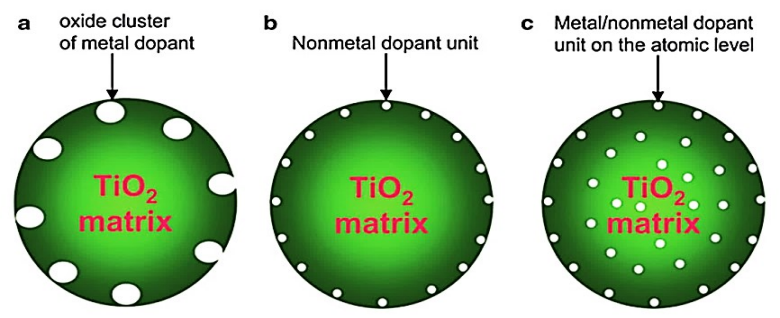

Figure 8. Different types of doping. similar way, metal ion ligand also has efficacy to convert $\mathrm{H}_{2} \mathrm{O}_{2}$ to hydroxyl ion due to the electron transfer mechanism in Fenton's reaction. Lee et al. tried to modify the surface of $\mathrm{WO}_{3}$ with iron, platinum, Nafion, and combination of these. The authors found the best combination to degrade the 4-chlorophenol was $\mathrm{WO}_{3} / \mathrm{Fe}(\mathrm{III}) / \mathrm{H}_{2} \mathrm{O}_{2}$ system [205].

\subsection{Chemical Modification}

Chemical modification (doping) is nothing but the addition of impurities, either it can be metal, non-metal, cation or anion to nanomaterial in order to enhance their potential and efficiency. The general importance of any nanomaterial lies in its good optical behavior. So, this property can be achieved by shifting of band gap from the ultraviolet region to the optical region and narrowing of band gap can be done by the doping. The main aim of doping is to increase the electrical and optical properties. Doping is the only method by which we can change the composition of nanocatalyst. Doping alters the morphological, magnetic, optical, structural and electrical properties of nano photocatalyst and also enhances the potential. Three types of doping are displayed in Figure 8. More than $40 \%$ of the photon comes under the visible light range covering large band area. The direct application of nanomaterial is quite impossible due to the activation of nanomaterial in the UV region. In this situation, a nonmetal comes into the picture to support the absorption capacity of nanocatalyst. Nonmetal co-doping has also synergistic advantages like

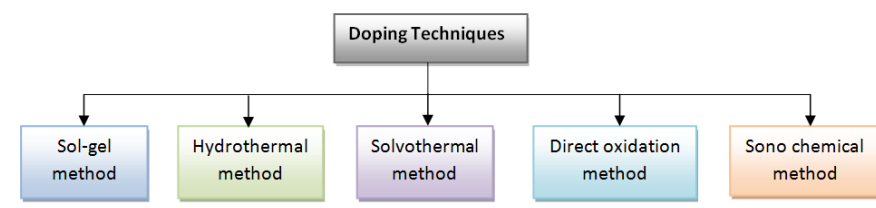

Figure 9. Various doping techniques.

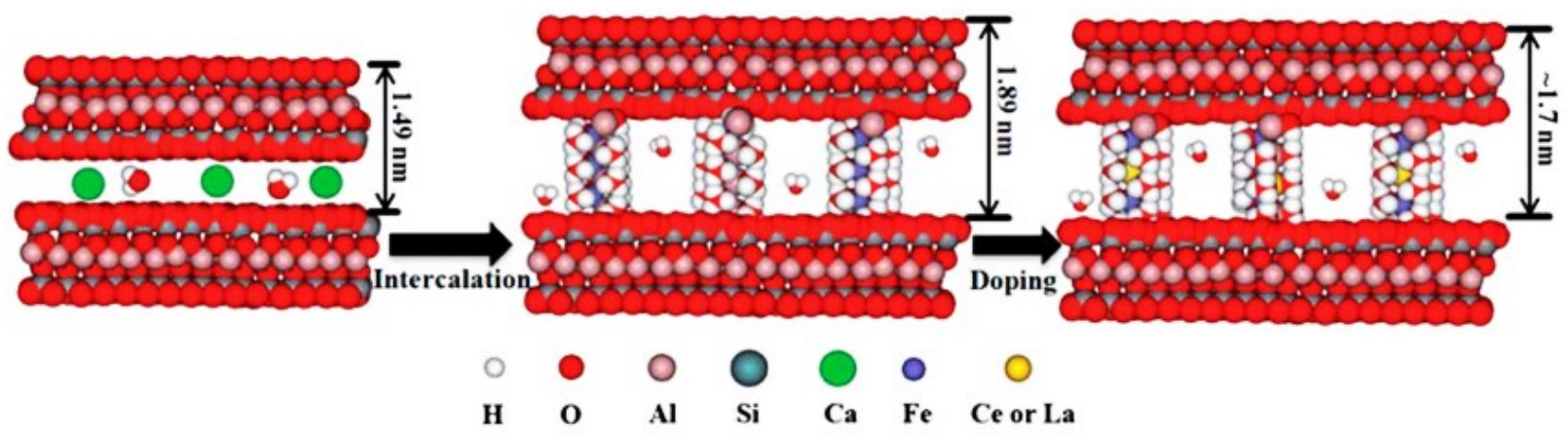

Figure 10. The insignificant doping. Ce or La was used as a doping material (Reprinted with permission from Ref. [206] copyright from RSC Advances). 
high visible responses, charge separation, etc. [3]. Various techniques for doping are shown in Figure 9. Sol-gel and hydrothermal method have already been discussed in the previous section.

In the solvothermal method, non-aqueous solvent can be applied and this is the only difference between hydrothermal and solvothermal methods. The higher temperature is used in solvothermal method compared with the hydrothermal method and it's a one of the major advantages of this process. Direct oxidation method is mostly applicable in the field of the fuel cell. Ultrasound has a great potential to the synthesis of high surface area nanomaterial. A small amount of doping is not that much significant as shown in Figure 10 [206]. Doping techniques cover the major area to prepare nanomaterial but it is not discussed in details here.

\section{Factors Affecting the Synthesis of Cata- lyst}

In this section, the effect of parameters, like $\mathrm{pH}$, calcination, and modification during catalyst synthesis and in treatment processes, will be discussed.

\subsection{Effect of $\mathrm{pH}$}

For the synthesis of catalyst, some researchers used acid medium and some researchers used alkali. It depends on the method of preparation and in precipitation method alkali medium is used. It can be judged by taking modifier or activator. Wang et al. demonstrated that acid modified catalyst is more efficient than alkali [66]. They investigated that during the acid wash, alkaline catalyst gets neutralized by acid and also observed the increased surface area and pore volume, i.e. higher exposed area for catalytic reaction. But in case of alkali modification, crystal structure is damaged due to the elemental disturbance in the catalyst. The alkaline catalyst increase the $\mathrm{pH}$ of wastewater which could also create some problem during the treatment of wastewater. Therefore, it is concluded that acid washing is an efficient process to increase the capacity of catalyst [68]. Liang et al. studied the effect of the precursor $\mathrm{pH}$ on catalyst structure as well as on photocatalytic performance. They found that $\mathrm{pH}$ is responsible to change morphology, structure, and property of catalyst. Less $\mathrm{pH}$ has strong visible light absorption capacity. Figure 11 (a) shows the influence of catalyst prepared at dif-

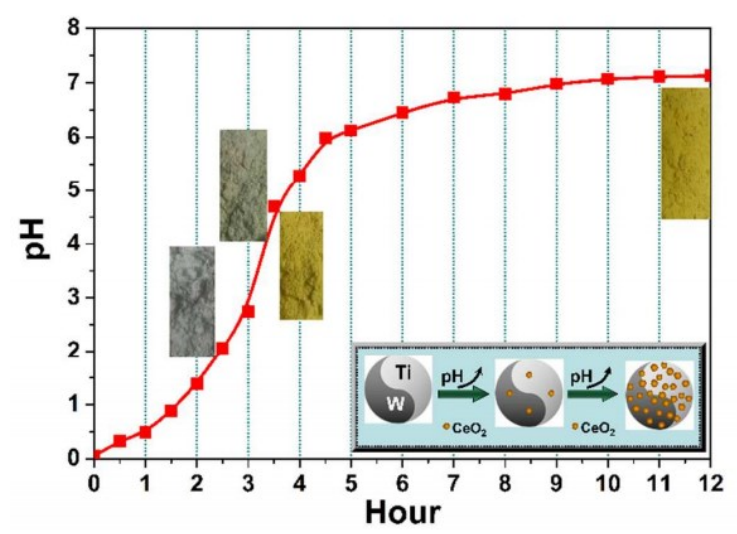

Figure 12. The synthesis of the catalyst at varying $\mathrm{pH}$ at different time by precipitation method [208] (Reprinted with permission from Ref. [208] copyright from RSC Advances).
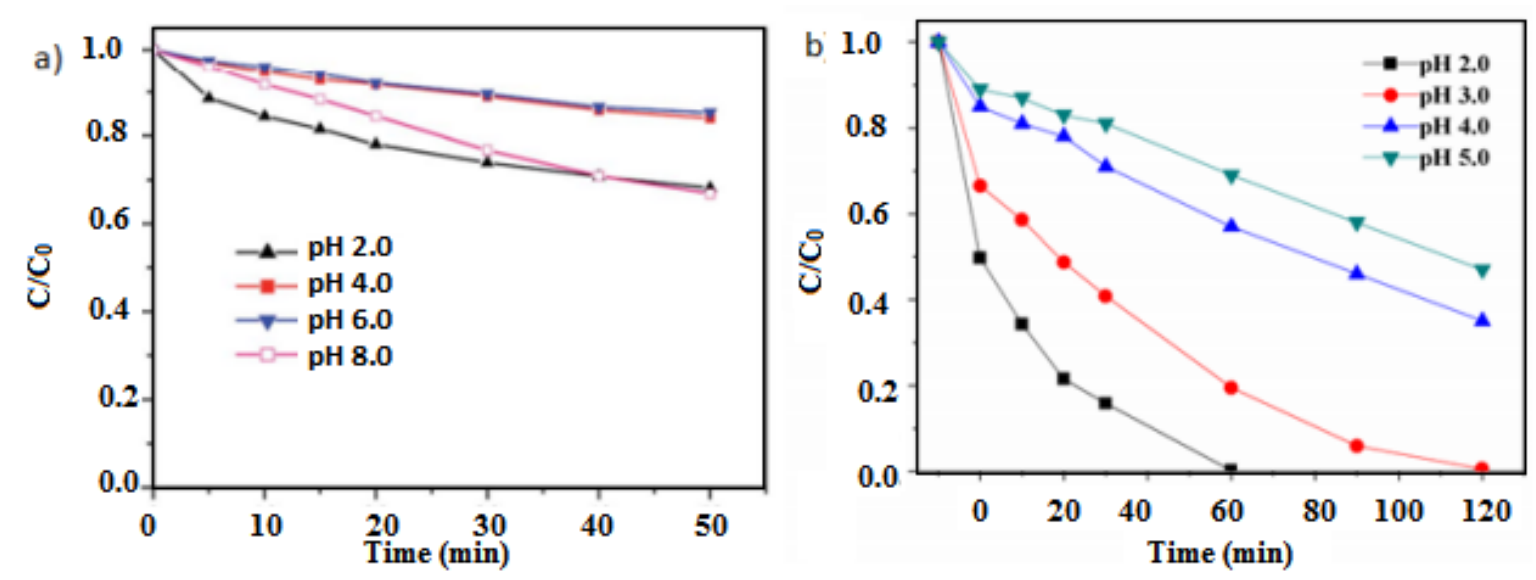

Figure 11. (a) The effect of different $\mathrm{pH}$ on the degradation of Orange II [207] (b) Catalytic performance of heterogeneous catalyst at different $\mathrm{pH}$ (Reprinted with permission from Ref. [209] copyright from RSC Advances). 
ferent $\mathrm{pH}$ on degradation of Orange II by photocatalytic activity. Catalyst prepared at $\mathrm{pH} 2$ exhibits higher degradation in lesser time as shown in Figure 11 (b) [206,207]. Huang et al. demonstrated that change in characteristics of the catalyst with a variation of $\mathrm{pH}$ at different times as shown in Figure 12. They found that with the increase of $\mathrm{pH}$ and time, color shifted from light yellow to yellow after 12 hours. $\mathrm{pH}$ can also affect the initiation, promotion, inhibition and direct reaction rate constant of any treatment processes [208,209]. Microscopic results showed that acidic medium can produce the smooth and regular structure. With the increase of $\mathrm{pH}$, the size of the particle also increases, but in basic medium size gets decreased [105].

\subsection{Effect of Calcinations}

In this section, the effect of temperature on catalyst properties as well as on treatment processes are discussed. Morphology, structure, crystallinity and surface area are significantly affected with the increase in temperature.

In case of $\mathrm{TiO}_{2}$ nanopowder, anatase phase powder was obtained in the temperature range of $250-500{ }^{\circ} \mathrm{C}$, while above $600{ }^{\circ} \mathrm{C}$ rutile phase powder was formed. But with the increase in temperature, surface area gets decreased. Anatase and rutile phase showed the different result in size distribution. The size of anatase phase powder was increased with the higher calcinations temperature, but in the case of ru- tile phase, size got increased very slightly [210,211]. Up to $300{ }^{\circ} \mathrm{C}$, there is no change in structure, crystallinity, and morphology. Crystallinity enhancement and impurities removal occur when calcinations temperature of material was increased to more than $300^{\circ} \mathrm{C}$. Due to this, photocatalytic activity is also increased [212].

The calcinations influence the reusability and hydrogenation capability of catalyst [213]. Zhang et al. investigated phenol degradation through the different calcined catalyst. In the range $400-600{ }^{\circ} \mathrm{C}$, catalytic performance was decreased due to the damage of internal voids. As a result, the availability of active sites becomes very less and decreases the active material in the solution. Therefore, it minimizes the degradation efficiency of the catalyst. Optimized calcinations of catalyst improves the adsorption capacity as well as increase the surface area by removing the unstable stratification [214]. Calcination is also responsible to maintain the rigidity. Wang et al. used three different temperatures to study the rigidity of metal coal fly ash. The optimized temperature was $723 \mathrm{~K}$ (Figure 13 (a)), as at this temperature pores have not been broken and along with that surface area was also increased with rigidity [66]. With the increase in calcinations temperature, evaporation occurs, gas produced and spread rapidly over the surface. As a result, removal of pollutant gets decreased which shows that the surface area and pore size get

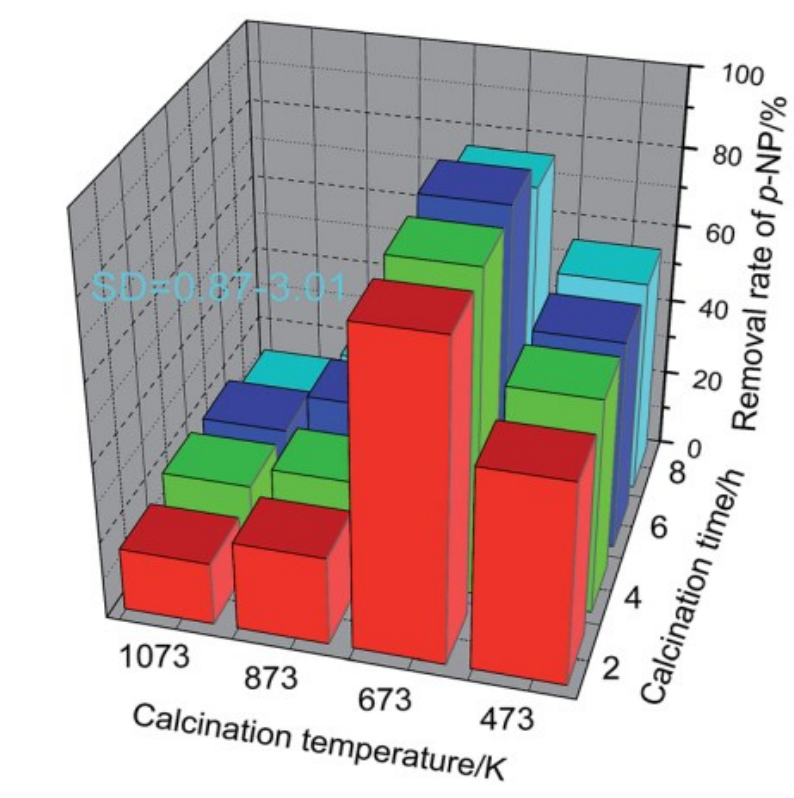

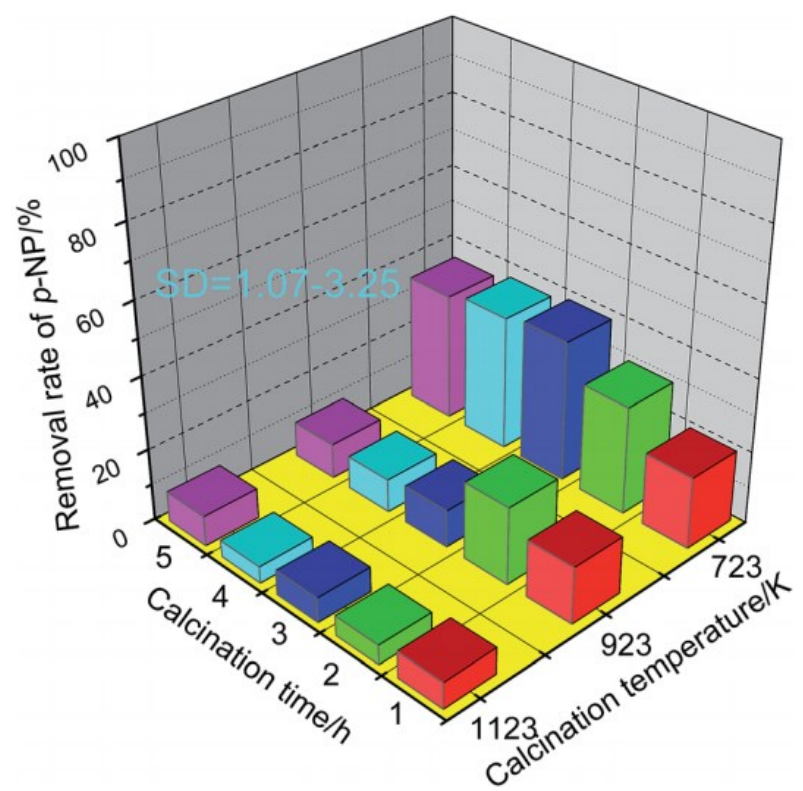

Figure 13. (a and b) The 3-dimensional view of the adsorptive and catalytic performance on the pollutant removal, with respect to calcinations time and temperature [68] (Reprinted with permission from Ref. [68] copyright from RSC Advances). 
affected. Therefore, adsorption capacity is decreased [68]. Figure 13 (b) displays catalytic performance at different calcination temperature. At low calcined catalyst, the stability of iron ion on the support material becomes weak and higher calcinations create a problem like loss of strengthening and uneven distribution of catalyst particles, etc.

On similar way, Wang et al. also worked on the phenol degradation and hydrogen peroxide decomposition with varying temperature. Calcinations at $550{ }^{\circ} \mathrm{C}$ was observed as optimized value for the degradation as well as decomposition of $\mathrm{H}_{2} \mathrm{O}_{2}$. Iron gets dissolved with the increase in temperature from 400 to $750{ }^{\circ} \mathrm{C}$, 0.698 to 0.149 , respectively. Effect of calcination can also be seen from XRD pattern which corresponds to structural alteration during phase change [58].

\subsection{Effect of Modifier}

Modifier plays a significant role on the preparation of the catalyst. The modifier can change the size, structure, stability, activity and surface area of the catalyst. There are various methods by which modifier can be applied in precipitation method, impregnation method, chemical vapor deposition method, hydrothermal method etc. It could be alkali metal, transition metal, zeolites or oxide form the element. Alipour et al. investigated that the addition of modifier decreases the surface area, but improves the activity and stability of the catalyst [215]. With the modifier, effective photodegradation efficiency was obtained compared to without modifier [216].

\section{Characterization of Synthesized Cata- lyst}

\subsection{SEM Analysis}

Scanning Electron Microscopy (SEM) images tell about the surface morphology of material which gives an idea about the structure (porous-non-porous, glossy, honeycomb) and shape (spherical) [68,217]. The surface morphology shows that all the constituents of the catalyst are distributed throughout without any major migration or phase segregation. The SEM images confirmed that pores are not significant in the synthesized catalyst [289]. This is one kind of microscopic analysis, which provides direct visual and reliable images. Figure $14(\mathrm{a}, \mathrm{b}, \mathrm{c}, \mathrm{d})$ is a demonstration of SEM images of iron-carbon xerogels with multi-dimensional and different sizes. Small incorporation of any other material and their effects can be visualized in the same figure (Figure 14 (b)). In addition, it also shows the rare or denser structure, smaller and larger particle size can also be seen when some new materials are added (Figure 14. c). Figure 14 (d) shows the image after polymerization which is highly dense and cross-linked structure [10].

Elemental analysis (atomic \% and weight \%) can be obtained when SEM connected with Energy Dispersive X-ray spectroscopy (EDX). EDX is also known as EDS, can be used to show or map one compound to another as shown in Figure 15 [7]. Generally, the magnification of any material through SEM can be in the range of $10^{5}-10^{6}$. For visualization of higher magnification, field emission-scanning electron microscopy (FESEM) is done. FESEM gives the knowledge of roughness or smoothness of material or particle [68]. Ramli et al. [86] reported that the method of preparation of catalyst can also change the morphology as shown in Figure 15 (d and e).

\subsection{XRD Analysis}

XRD is one of the most important instruments to reveal the crystallographic information. XRD analysis indicates the primary phase in the catalyst and the diffraction lines remain unchanged by the addition of impreg-

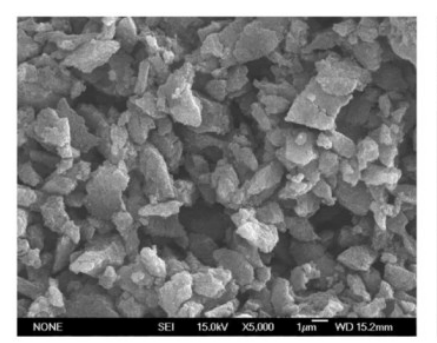

(a)

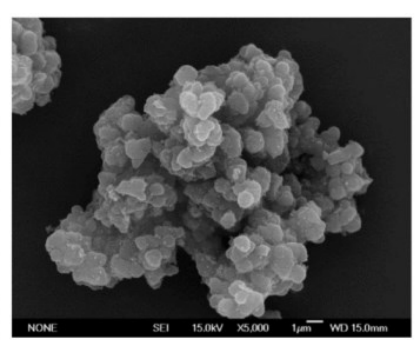

(b)

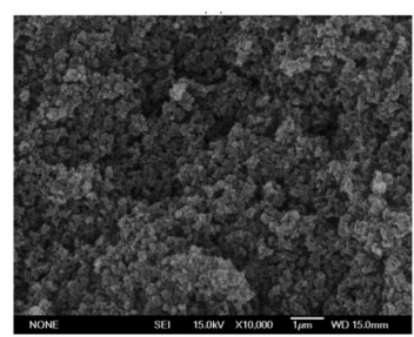

(c)

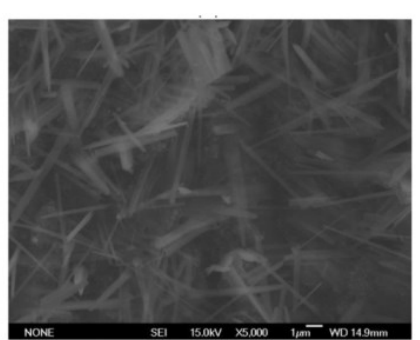

(d)

Figure 14. (a,b,c,d): The formation of iron-carbon xerogel [38]. The sizes of microbeads are $3 \mu$ and 650-830 $\mu$ with respect to (a) and (b) (Reprinted with permission from Ref. [10] copyright from Elsevier). 


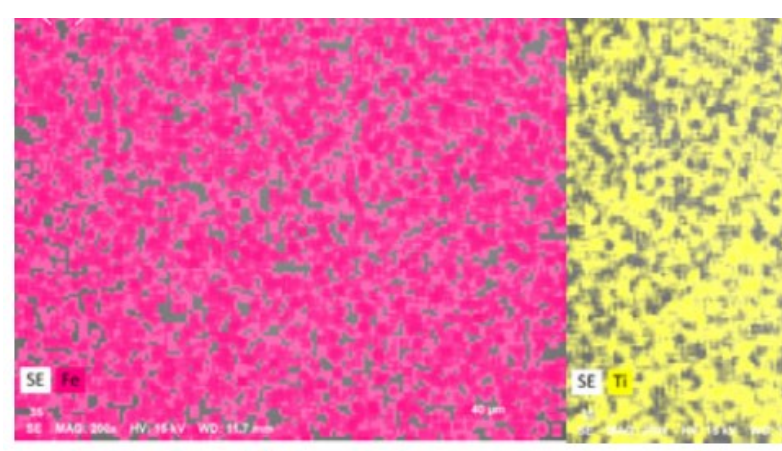

a)

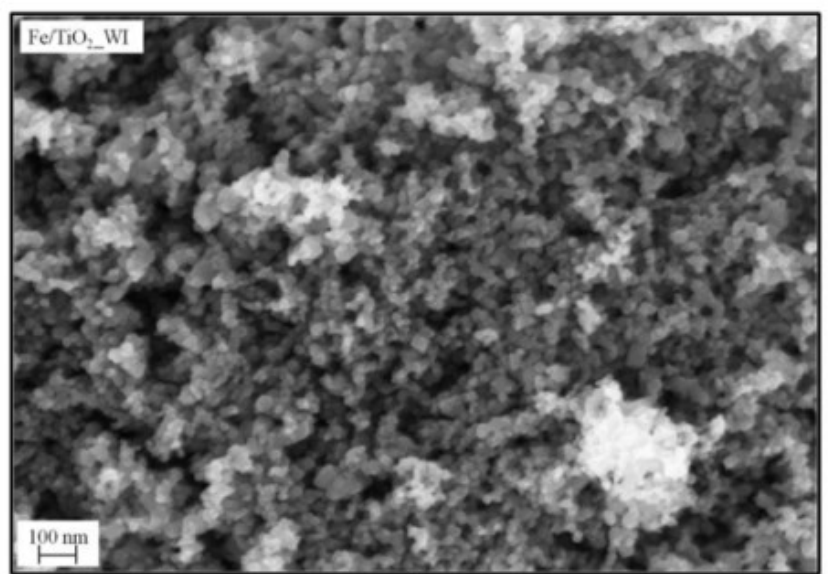

d) b)

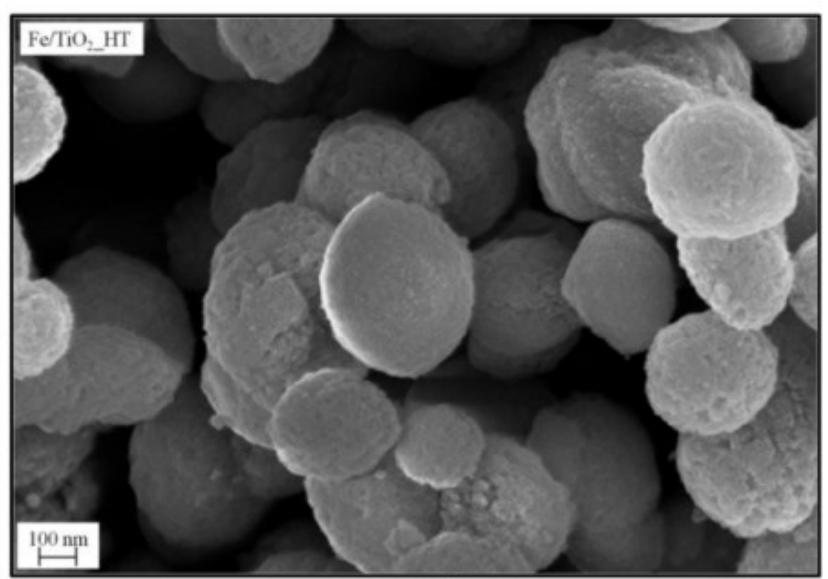

e)

Figure 15. The red color showing iron (a) and yellow color showing is Titanium (b). Red color distributed over Titanium (c) [7]. Figure $d$ and e illustrate the morphology of catalyst $\left(\mathrm{Fe} / \mathrm{TiO}_{2}\right)$ synthesized by wet impregnation and hydrothermal method respectively with $50 \times 10^{3}$ magnification (Reprinted with permission from Ref. [7] copyright from Elsevier).
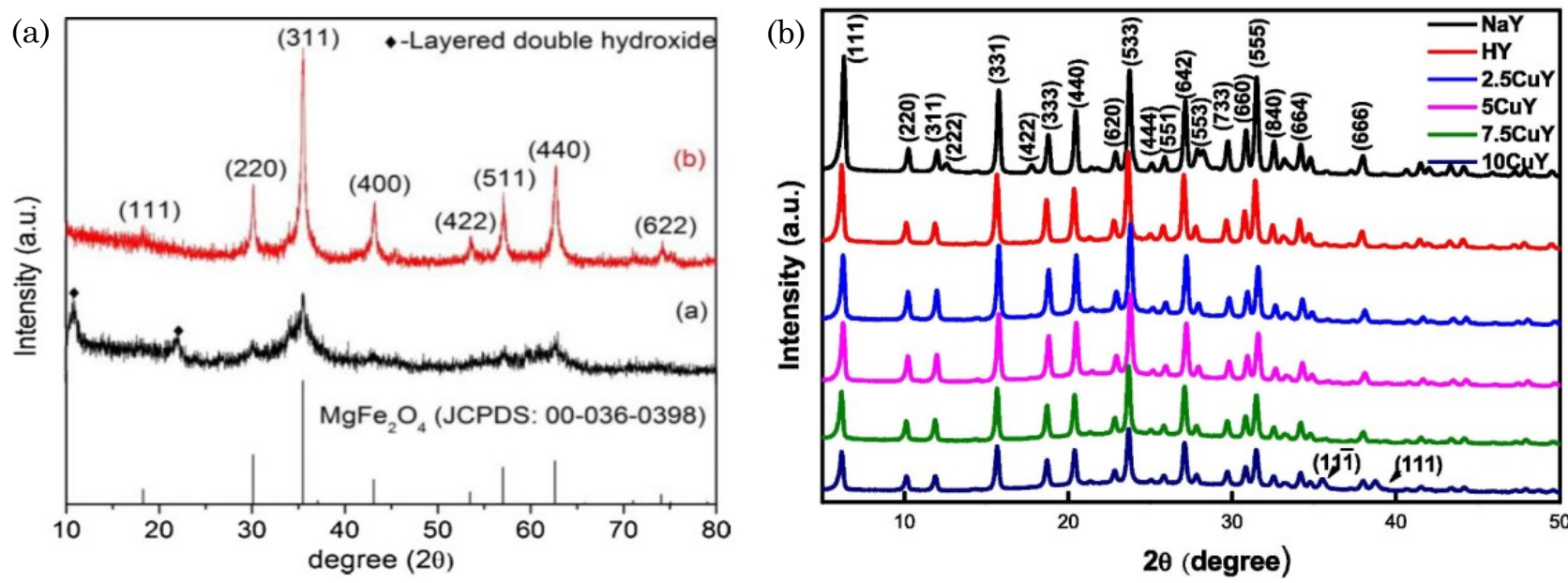

Figure 16. (a) The XRD pattern of the prepared catalyst by hydrothermal method. The black and red pattern shows the before and after calcination $\left(550^{\circ} \mathrm{C}\right.$ for $2 \mathrm{~h}$ ). Before calcination (black pattern) only four peaks were found at the angle of 30.1 (220), 35.5(311), 57.0(511), 62.6(440) and two more peaks. After calcination, a red pattern shows highly enhanced peaks [58]. (b) XRD patterns of fresh CuY zeolite with different loading [77]. Reprinted with permission from Ref. [77] copyright from Elsevier (Reprinted with permission from Ref. [58] copyright from Elsevier). 
Table 7. Wavenumber, characteristics and functional group of catalyst.

\begin{tabular}{|c|c|c|}
\hline Wavenumber $\left(\mathrm{cm}^{-1}\right)$ & Characteristics and functional group & References \\
\hline $412-400,597-615,478$ & $\mathrm{M}-\mathrm{O}$ & {$[228,229]$} \\
\hline 670 & $\mathrm{O}-\mathrm{H}$ & [229] \\
\hline $700-1300,790$ & C-H bending vibration & {$[105,127]$} \\
\hline $875-750$ & $\mathrm{C}-\mathrm{H}$ & [230] \\
\hline 919 & $\mathrm{~N}-\mathrm{O}$ & [231] \\
\hline 1020,1014 & C-O stretching & [229] \\
\hline 1030 & $-\mathrm{OH}$ & [232] \\
\hline 1051,1117 & Sulphate $\left(\mathrm{SO}_{4}{ }^{2-}\right)$ & [233] \\
\hline 1060 & $\mathrm{~T}-\mathrm{O}(\mathrm{T}=\mathrm{Si}, \mathrm{Al})$ & [234] \\
\hline 1200,1400 & $\mathrm{Si}-\mathrm{O}-\mathrm{Si}$ & [235] \\
\hline 1211 & $\mathrm{C}-\mathrm{O}-\mathrm{C}$ & [236] \\
\hline $1000-1450$ & $\mathrm{C}-\mathrm{O}$ & [230] \\
\hline 1350 & Stretching vibration of $\mathrm{NO}_{3}$ & [237] \\
\hline 1352 & $-\mathrm{NH}$ & [238] \\
\hline 1384 & C-N stretching & [229] \\
\hline $1396,1424-1429,1430$ & $\begin{array}{l}\mathrm{R}-\mathrm{H} \text {, symmetric and asymmetric carboxyl } \\
\text { group }\end{array}$ & {$[227,232,239]$} \\
\hline 1450 & Benzene & [240] \\
\hline 1400,1650 & $\mathrm{H}-\mathrm{OH}$ bending vibration & [235] \\
\hline 1570 & $\mathrm{H}-\mathrm{O}-\mathrm{H}$ absorption & [61] \\
\hline $\begin{array}{l}1615-1580,1510-1450,1680- \\
1620,1633,1515-1520,1571\end{array}$ & $\mathrm{C}=\mathrm{C}$ stretching & {$[229,230,233,236,240]$} \\
\hline $1635-1570$ & $\mathrm{C}=\mathrm{O}$ & [241] \\
\hline 1618,1563 & Amide I and amide II & [242] \\
\hline $1630-1640,1703$ & $\begin{array}{l}\text { O-H bending vibration due to adsorbed } \\
\text { water molecules at the catalyst surface }\end{array}$ & {$[227]$} \\
\hline 1647 & $\mathrm{C}=\mathrm{N}$ & [231] \\
\hline $\begin{array}{l}1658,1640-1650,1700,1680- \\
1687\end{array}$ & $\mathrm{C}=\mathrm{O}$ & {$[230,237,243,244]$} \\
\hline 1731 & Urethane & [242] \\
\hline $1739-1775$ & $\mathrm{O}-\mathrm{H}$ stretching and bending vibration & [227] \\
\hline 2098 & $\mathrm{C} \equiv \mathrm{N}$ & [245] \\
\hline 2300 & Isocyanate & [242] \\
\hline 2243 & Nitrile & [231] \\
\hline $2800-3750$ & $\mathrm{OH}$ group & [235] \\
\hline 2839 & Aliphatic C-H stretching vibration & [233] \\
\hline $\begin{array}{l}2922,3014, \\
2940,2924,2847,2925\end{array}$ & Aromatic C-H stretching vibration & {$[105,232,233,246]$} \\
\hline $\begin{array}{l}3400,3420-3450,3570,3573- \\
3574,3777,3430\end{array}$ & $\mathrm{O}-\mathrm{H}$ stretching and bending vibration & {$[61,105,227,232]$} \\
\hline $3700-3000$ & $\mathrm{O}-\mathrm{H}, \mathrm{N}-\mathrm{H}$, water molecule & {$[231,239]$} \\
\hline
\end{tabular}


nants using co-precipitation method for preparation of any catalyst [289]. Scherrer equation is used for determining the crystals size. Sometimes XRD pattern is also utilized for the quantitative investigation and detection purposes [218,219]. X-ray diffraction is not only used for the identification of structural change during phase transformation, but also to check the purity and improvement of catalyst (as shown in Figure 16 (a) [58]. Some studies reported that due to coating or burning of carbon nanotubes, peak decreases and get broad [139]. Broadening of peaks shows poor crystallinity and nonstructural dimensions [220]. Broadening or disappearing of peaks is due to the calcination of the sample. During calcination, catalyst is directly exposed to temperature and air, hence hydrolysis occurs and local sites gets affected. Few composites do show diffraction pattern or line which means that composite is in the amorphous state $[221,222]$. Due to the modification of catalyst, either by acid treatment, alkali treatment, heat treatment $[58,118]$ or metal loading [77], diffraction pattern changes. Authors investigated that when copper loaded from 2.5 to $10 \mathrm{wt} \%$, peaks decrease from $100 \%$ to $72 \%$ and less ordered structure are formed (as shown in Figure 16 (b) [77]. Holding time is not much more significant to change the crystal structure during calcination reported by $\mathrm{Hu} \&$ co-researchers $[118,223]$. Any catalyst prepared by different methods (wet impregnation, hydrothermal method, etc.) can alter the structure of catalyst which can be observed by change in peaks [86]. XRD also reveals the impurities present in the catalyst or unreacted molecule. For calculation of particle size through XRD pattern, following formula (Williamson formu-

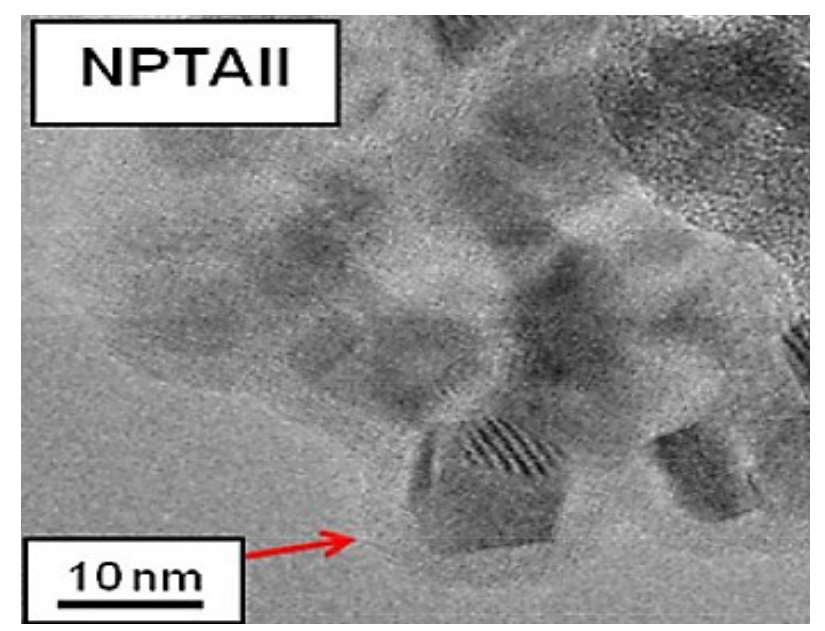

Figure 17. HRTEM image (Reprinted with permission from Ref. [253] copyright from Elsevier). la) can be used [224]:

$$
\beta \cos \theta / \lambda=1 / L+\varepsilon \sin \theta / \lambda
$$

where, $\beta=$ full-width half maximum of diffraction peak, $\varepsilon=$ strain, and $L=$ crystalline size.

\subsection{FTIR Analysis}

The synthesized catalysts are examined using FTIR for the identification of interactions between the functional groups during catalyst preparation. Fourier transform infrared spectroscopy instrument is generally used to characterize the catalyst in terms of availability of functional groups like carboxyl $(-\mathrm{COOH})$, a hydroxyl group (C-OH), epoxide (C-O-C), lactone etc. and associated chemical bonding [225,226]. In the investigation range functional group varies from wavenumber 400 to $4000 \mathrm{~cm}^{-1}$ [227]. FTIR value shows the bending or stretching of the bond between molecule or atoms. In present days, researchers use FTIR to analyze the peaks (broadening and narrowing of peaks) before and after the reaction to check the stability of catalyst [144]. The Table 7 shows the wavenumber and their respective functional groups.

\subsection{TEM, HRTEM, STEM Analysis}

Transmission Electron Microscopy (TEM) is used to elemental mapping, distribution or metallic dispersion of particle in the hybrid form of material. Uniform distribution of particle has significant catalytic efficiency [52,247]. TEM images are also used to determine the particle shape, size, and morphologies of particles [248-250]. High-Resolution Transmission Electron Microscopy (HRTEM) finds the microstructure and morphology of synthesized catalyst. HRTEM images reveal the extensive interface interaction between metal oxides like $\mathrm{Fe}_{3} \mathrm{O}_{4}, \mathrm{Mn}_{3} \mathrm{O}_{4}$, etc. [95,251]. It also differentiates the amorphous and crystal structure of particle (crystal is encapsulated with amorphous layer) as shown in the Figure 17 in red arrow [252]. Scanning transmission electron microscopy (STEM) shows the distribution of metal ions on support or matrix [253].

\subsection{BET Analysis}

BET (Brunauer-Emmett-Teller) analysis is used to study of the specific surface area of the catalyst. Different catalyst synthesis process could affect the characteristics and properties of the catalyst due to their varying surface area, i.e. it can control the catalytic behavior of 
catalyst. The determination of surface area is directly related with the formation, deformation of bond or structure due to the thermal process, catalyst poisoning and also with catalytic performance. Catalytic activity will be high with higher surface area and with smaller pore volume [164]. Three methods are available to measure the surface area of any catalyst: volumetric, gravimetric, dynamic, but the commonly used method is the volumetric method based on nitrogen adsorption at fixed time and temperature [254]. With the analysis of BET, the total pore volume $\left(p / p_{0}=0.98\right)$, average pore width $\left(W=4 . V_{\mathrm{mic}} / S_{\mathrm{mic}}\right)$ and the dia. of the catalyst using the Barrett-Joyner-Halenda (BJH) model can be determined [255].

\subsection{Thermogravimetric Analysis (TGA)}

Thermogravimetric Analysis (TGA) is conducted on powder to see the changes in weight with the changes in temperature. The changes in weight are correlated with the sample composition and stability. This analysis is extensively utilized to measure the thermal stability, composition, moisture, oxidative stability, volatile content, kinetics of decomposition, sample dehydration and lifetime. Schematic diagram of the TGA instrument is illustrated in Figure 18 [254]. Weight loss of the prepared catalyst in different temperature region is noted. Mostly, the first weight loss occurs at around $150{ }^{\circ} \mathrm{C}$ due to absorption of water molecules on the catalyst surface $[164,283]$. The weight loss above $500{ }^{\circ} \mathrm{C}$ is due to the oxidation loss $[284,285]$.

\subsection{Raman Spectroscopy}

This instrument is used to know the crystal formation or ordered or disordered structure [256]. There are two important Raman peaks:

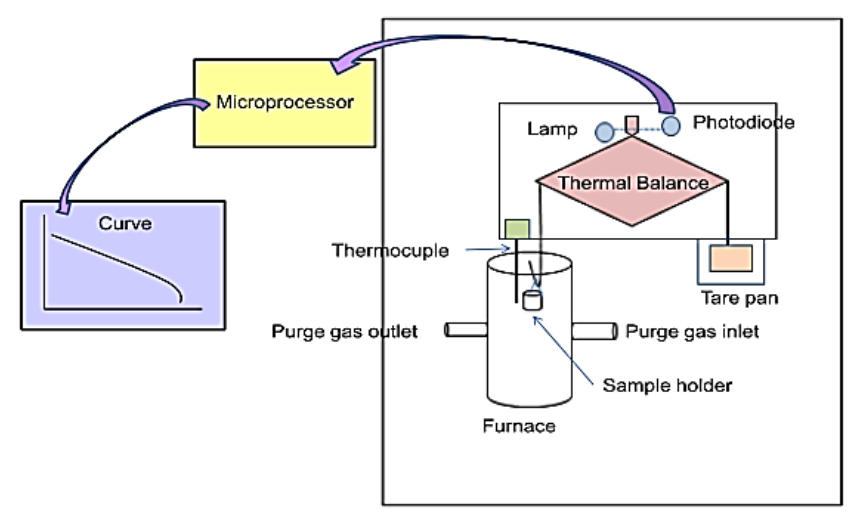

Figure 18. Schematic diagram of TGA instrument.
$\mathrm{D}$ band related to the disorders in the wall of catalyst and G band related to the C-C stretching mode of carbon, the intensity ratio of $\mathrm{D}$ and $\mathrm{G}$ band shows the degree of disorder in the walls of the catalyst [286-289]. An et al. used this technique to analyze the calcined catalyst (graphene $-\mathrm{BiFeO}_{3}$ ) before and after the reaction and at the time of synthesis at $600{ }^{\circ} \mathrm{C}$. Broadening and shifting of peaks in Raman Spectroscopy curve tells about the addition or doping of metal [150]. It is one of the popular vibrational spectroscope apart from FTIR which can be utilized to assess the motion of the molecule, identification of species and functional group [254].

\subsection{Zeta Potential Measurement-Isoelectric Point (PZC- Point of Zero Charges)}

Particle size distribution profile of suspended particles was determined using the dynamic light scattering (DLS) technique. The zeta potential indicates the degree of repulsion between adjacent charged particles in the dispersion and thus relate to the stability of the dispersion. At lower absolute zeta potential, particles get agglomerated, while resisting agglomeration at high absolute zeta potential. Zeta potential is used to determine the stability of catalyst at different $\mathrm{pH}$. Zeta potential is nothing but a charge (in $\mathrm{mV}$ ) present in the catalyst surface [257]. Zeta potential (马) and Isoelectric point (PZC) are related to each other. Zeta potential always decreases with the increase of $\mathrm{pH}$. High zeta value of any catalyst means high stability. At low $\zeta$, attraction exceeds repulsion and dispersion will break and flocculate [217]. Similar result was found by Cao et al. at $\mathrm{pH}$ of 10 , low $\zeta$ value (-3.4) was obtained, but with the decreasing $\mathrm{pH}$ ( $\mathrm{pH}$ from 3 to 9 , the $\zeta$ values were $33.7 \mathrm{mV}, 25.5 \mathrm{mV}, 18.9 \mathrm{mV}, 4.52 \mathrm{mV}$, respectively), significant $\zeta$ value found [258]. According to Hogland et al. highest proportion of micropores have low $\zeta$ and PZC. This is due to the presence of high oxygen-containing functional groups [259]. An et al. reported that the catalyst activation ability decreases at high $\mathrm{pH}$ [256].

\subsection{X-ray Photoelectron Spectroscopy (XPS)}

XPS techniques are used to analyze the top surface layer and find the composition of material with electronic binding energy of prepared catalyst. The electron binding energy is calculated by the following formula:

$$
E_{\mathrm{k}}=h v-E_{\mathrm{b}}-\Phi
$$


where $E_{\mathrm{k}}$ is the kinetic energy of emitted photoelectron, $h v$ is photo energy, $\Phi$ is work function, and $E_{\mathrm{b}}$ is binding energy.

The main advantages of XPS are to identify the chemical state on the surface and to differentiate the oxidation states of present molecules on the surface. It can identify the atomic concentration of $\mathrm{O}, \mathrm{C}, \mathrm{S}$, and $\mathrm{Fe}$ on the catalyst surface. In Fenton process, the importance of XPS lies in the oxidation state of $\mathrm{Fe}$ on the catalyst surface which can be identified by this techniques and oxygen status also plays a vital role in oxidation process. There are four peaks under oxygen region, i.e. chemisorbed oxygen, lattice oxygen, chemically or physically adsorbed water. According to literature, chemisorbed oxygen plays an active role in oxidation process and increases the catalytic activity [46].

The binding energy is calculated only for the particular element [57,260]. Some researchers used XPS before and after the reaction and they found the regeneration of species and identified active species [21,258]. XPS results also validate the significant fabrication of catalyst [57].

5.10 Temperature Programmed Reduction Profiles (TPR)

TPR gives the knowledge about the nature of catalyst (reduction with temperature) when matrixed with supportive or other materials. This technique also gives the information about how any catalyst is formed/reduced in any composite surface with the varying percentage at a different temperature. Rezende et al. synthesized a catalyst of varying iron $\%$ with niobium and found the important information about iron reduction with temperature $\left(\mathrm{Fe}_{2} \mathrm{O}_{3} \rightarrow \mathrm{Fe}_{3} \mathrm{O}_{4} \rightarrow \mathrm{FeO} \rightarrow \mathrm{Fe}\right.$ at different temperature $430{ }^{\circ} \mathrm{C}, 640{ }^{\circ} \mathrm{C}, 800{ }^{\circ} \mathrm{C}$ ). They also revealed that the sample of $2.4 \% \mathrm{Fe} / \mathrm{Nb}$ at $600{ }^{\circ} \mathrm{C}$ with intensive peaks have excellent thermal stability and $7.6 \% \mathrm{Fe} / \mathrm{Nb}$ is more reactive [261].

\subsection{Diffuse Reflectance Spectroscopy (DRS)}

The UV-Vis DRS technology is used to find the band gap energy of any catalysts. It also reveals the UV and visible light absorption capacity of any catalyst when composites are coupled. The band gap decreases with increase in Fe concentration in the catalyst, it may be closer to pure Fe-oxide. An et al. found that graphene- $\mathrm{BiFeO}_{3}(1.0 \mathrm{ev})$ have more light absorption capacity comparatively $\mathrm{BiFeO}_{3}(2.05 \mathrm{ev})$ [256]. A similar investigation was done by some authors. They found that $\mathrm{Fe}_{3} \mathrm{O}_{4} / g-\mathrm{C}_{3} \mathrm{~N}_{4}$ has high absorption capacity compared with g$\mathrm{C}_{3} \mathrm{~N}_{4}$ [262].

\section{Application of Synthesized Catalyst in the Fenton Process}

Main utilization of synthesized catalyst in the Fenton process is to treat synthetic and in-

Table 8. Application of synthesized catalyst in real wastewater.

\begin{tabular}{|c|c|c|c|c|c|c|c|}
\hline \multirow{2}{*}{ Effluent type } & \multirow{2}{*}{ Catalyst } & \multirow{2}{*}{$\begin{array}{c}\text { Catalyst preparation } \\
\text { method }\end{array}$} & \multicolumn{4}{|c|}{$\begin{array}{c}\text { Treatment/Removal/ } \\
\text { Degradation }\end{array}$} & \multirow{2}{*}{ Ref. } \\
\hline & & & $\mathrm{COD}$ & Color & TOC & BOD & \\
\hline Cosmetic industry & $\begin{array}{l}\mathrm{Fe} / \mathrm{Al}_{2} \mathrm{O}_{3} \text { and } \\
\mathrm{Fe} / \mathrm{AC}\end{array}$ & $\begin{array}{l}\text { Incipient wetness im- } \\
\text { pregnation method }\end{array}$ & $85 \%$ & - & $55 \%$ & - & {$[263]$} \\
\hline $\begin{array}{l}\text { Pharmaceutical in- } \\
\text { dustry }\end{array}$ & $\mathrm{Fe}-\mathrm{TiO}_{2}$ & Dip coating method & $83 \%$ & - & - & - & {$[264]$} \\
\hline $\begin{array}{l}\text { Household (sewage } \\
\text { wastewater) }\end{array}$ & $\mathrm{Fe} / \mathrm{C}$ & - & $75 \%$ & - & - & - & {$[265]$} \\
\hline Leather industry & MAC & - & $69 \%$ & & $61 \%$ & $74 \%$ & {$[266]$} \\
\hline Fertilizer industry & $\mathrm{Fe} / \mathrm{TiO}_{2}$ & Deposition-precipitation & - & - & $96 \%$ & - & {$[267]$} \\
\hline Oil refinery & $\begin{array}{l}\text { Fe-pillared } \\
\text { bentonite }\end{array}$ & ion exchange & $92 \%$ & - & - & - & {$[268]$} \\
\hline Pulp and Paper & $\mathrm{Fe}-\mathrm{Mn} / \mathrm{NaY}$ & $\begin{array}{l}\text { Impregnation } \\
\text { method/sol-gel }\end{array}$ & $\begin{array}{c}75.2 \% / \\
45 \%\end{array}$ & - & - & - & {$[269]$} \\
\hline Landfill leachates & $\mathrm{GAC} / \mathrm{Fe}$ & Impregnation method & $95 \%$ & $93 \%$ & - & - & {$[270]$} \\
\hline Textile industry & $\mathrm{Fe} / \mathrm{AC}$ & Impregnation method & $66.3 \%$ & $96.7 \%$ & $73.6 \%$ & $72.5 \%$ & {$[271]$} \\
\hline
\end{tabular}


Table 9. Application of synthesized catalyst on dye effluent.

\begin{tabular}{|c|c|c|c|c|c|}
\hline Dye & Catalyst & $\begin{array}{l}\text { Catalyst prepara- } \\
\text { tion method }\end{array}$ & Degradation & Remark & Ref. \\
\hline Methylene blue & $\mathrm{CoMoO}_{4}$ & Hydrothermal & $100 \%$ & $\begin{array}{l}\text {-High surface area= } 61.9 \\
\mathrm{~m}^{2} / \mathrm{g} \text {. } \\
\text {-Universality. }\end{array}$ & {$[210]$} \\
\hline $\begin{array}{l}\text { Reactive Red } \\
120\end{array}$ & Fe-ZSM-5 & $\begin{array}{l}\text { Hydrothermal and } \\
\text { Impregnation }\end{array}$ & $98 \%$ & $\begin{array}{l}\text {-High crystallinity, and sur- } \\
\text { face area. } \\
\text {-Reusable nano catalyst. }\end{array}$ & {$[272]$} \\
\hline $\begin{array}{l}\text { Basic violet } 1 \\
\text { and Basic } \\
\text { green } 4\end{array}$ & $\mathrm{Cu}-\mathrm{ZnO}$ & Wet-Impregnation & $\begin{array}{c}95.5 \% \text { and } \\
100 \%\end{array}$ & $\begin{array}{l}\text {-Promising sonophotocatalyst } \\
\text { and excellent reusability. }\end{array}$ & {$[273]$} \\
\hline $\begin{array}{l}\text { RB-21, RR-141 } \\
\text { and RG-6G }\end{array}$ & $\mathrm{CZrW} / \mathrm{ZrW}$ & Hydrothermal & $\begin{array}{c}95 \%, 92 \% \text { and } \\
50 \%\end{array}$ & $\begin{array}{l}\text { - } \mathrm{CZrW} \text { is best found for deg- } \\
\text { radation while } \mathrm{ZrW} \text { was best } \\
\text { for mineralization. }\end{array}$ & {$[274]$} \\
\hline $\begin{array}{l}\text { Reactive Blue } \\
52\end{array}$ & Fe-doped $\mathrm{TiO}_{2}$ & $\begin{array}{l}\text { Microwave assist- } \\
\text { ed hydrothermal }\end{array}$ & $100 \%$ & $\begin{array}{l}>4 \text { the release of iron in solu- } \\
\text { tion was found negligible. }\end{array}$ & {$[275]$} \\
\hline Methyl Orange & $\mathrm{NdFeB}-\mathrm{AC}$ & $\begin{array}{l}\text { Negative pressure } \\
\text { Impregnation } \\
\text { method }\end{array}$ & $97.8 \%$ & $\begin{array}{l}\text {-Catalyst has good applica- } \\
\text { tion prospect with high sta- } \\
\text { bility. } \\
\text {-Catalytic degradation shows } \\
\text { pseudo } 1^{\text {st }} \text { order model. }\end{array}$ & [88] \\
\hline Methyl green & $\mathrm{CeCX}$ & Impregnation & $100 \%$ & $\begin{array}{l}\text {-Catalyst show the synerget- } \\
\text { ic effect (adsorption and oxi- } \\
\text { dation) }\end{array}$ & {$[276]$} \\
\hline $\begin{array}{l}\text { Methylene } \\
\text { Blue }\end{array}$ & $\begin{array}{l}\mathrm{Fe}_{3} \mathrm{O}_{4} / \mathrm{SiO}_{2} / \mathrm{C} \\
\text { nanoparticles } \\
(\text { FSCNP) }\end{array}$ & Hydrothermal & $94 \%$ & $\begin{array}{l}\text {-Catalyst has large } \mathrm{pH} \\
\text { range, fast kinetics, good } \\
\text { resistance against scaven- } \\
\text { gers' radicals }\end{array}$ & {$[277]$} \\
\hline Reactive Red 2 & $\mathrm{CuFe}_{2} \mathrm{O}_{4}$ & Co-precipitation & $91.3 \%$ & $\begin{array}{l}\text {-Catalyst show reusability, } \\
\text { applicability and high stabil- } \\
\text { ity. } \\
\text { - NCFOH show high applica- } \\
\text { bility than CFOH. }\end{array}$ & {$[100]$} \\
\hline Rhodamine B & $\mathrm{Fe}_{2}\left(\mathrm{MoO}_{4}\right)_{3}$ & $\begin{array}{l}\text { Co-precipitation } \\
\text { and sol gel }\end{array}$ & $97 \%$ & $\begin{array}{l}\text {-Synergistic effect of } \mathrm{Fe}^{3+} \text { and } \\
\mathrm{MoO}_{4} 2^{-} \\
-\mathrm{k}_{\text {co-precipitation }}=1.5 \mathrm{k}_{\text {solgel }}\end{array}$ & [30] \\
\hline Orange II & $\mathrm{MeSrCuO}$ & Sol gel & $97 \%$ & $\begin{array}{l}-\mathrm{Cu}^{2+} \text { was active phase to } \\
\text { degrade O II. }\end{array}$ & {$[278]$} \\
\hline $\begin{array}{l}\text { Methylene } \\
\text { Blue }\end{array}$ & $\begin{array}{l}\text { Kieselguhr/ } \\
\mathrm{Fe}_{2} \mathrm{O}_{3} / \mathrm{TiO}_{2}\end{array}$ & $\begin{array}{l}\text { Co-precipitation } \\
\text { and impregnation }\end{array}$ & $98.86 \%$ & $\begin{array}{l}\text {-Ecofriendly catalyst. } \\
\text {-Easy recovery by external } \\
\text { magnetic field. }\end{array}$ & {$[279]$} \\
\hline Rhodamine B & Fe/SBA-15 & Impregnation & $93 \%$ & $\begin{array}{l}\text {-Easily regenerated by soak- } \\
\text { ing with } \mathrm{H}_{2} \mathrm{O}_{2} \text {. } \\
\text {-Less leaching of iron and } \\
\text { highly stable. }\end{array}$ & {$[280]$} \\
\hline $\begin{array}{l}\text { Methyl Orange } \\
\text { and Rhoda- } \\
\text { mine B }\end{array}$ & $\mathrm{BaNaPW}$ & Hydrothermal & $>95 \%$ & $\begin{array}{l}\text {-Bisfunction (photocatalyst } \\
\text { and flocculant) }\end{array}$ & [99] \\
\hline $\begin{array}{l}\text { Methylene blue } \\
\text { and Rhoda- } \\
\text { mine B }\end{array}$ & $\mathrm{Gr}-\mathrm{Fe} / \mathrm{Ce}$ & Hydrothermal & $>90 \%$ & $\begin{array}{l}\text {-Ce plays a key role to lower } \\
\text { the band gap. }\end{array}$ & {$[281]$} \\
\hline
\end{tabular}


dustrial wastewater. Currently, many types of wastewater are generated from various sectors that can be treated by Fenton process. Various researches already have been done with catalytic Fenton process, which are listed below in Table 8 and Table 9, represent the industrial wastewater contaminated with different dyes.

The evolution over the last two decades of heterogeneous catalyst preparation method, modification, characterization and overall application has been discussed. Heterogeneous catalysts have various advantages such as high surface area, less leaching of iron, less sludge formation, high recycling possibilities, ease in separation and faster rate of reaction which are useful for the suitable applications in the field of wastewater treatment using Fenton's process. The focus of this review is on the synthesis methods, physical and chemical modification to enhance their efficacy, factors which may possibly affect the performance of catalyst during synthesis, characterization such as SEM, XRD, TEM, PZC, TPR, DRS and applications.

The fundamentals of catalyst synthesis are extensively described in this review and the readers will get benefited from the deep insights. Among four synthesis methods, precipitation method was found to be most popular and used method based on Scopus data source.

The efficiency of catalyst can be enhanced by modification of catalyst and that modification is related to the changes in surface morphology and addition of foreign materials. Suitable shape and dimension of any synthesized catalyst is important to be effective catalyst. Diversified characteristics of catalyst can be achieved by only modification. Modification (doping) of catalyst enhances the morphological, magnetic, optical, structural and electrical properties. Small doping is not always fruitful for effective potential of catalyst at the same time. Ultrasound technique has a vital role to enhance the surface area of catalyst.

Low $\mathrm{pH}$ modified catalyst may have an excellent potential comparatively higher $\mathrm{pH}$ modified catalyst. It always enhances the catalytic efficiency. Calcination temperature may affect the characteristics and efficiency of catalyst. It also affects the reusability and hydrogenation of catalyst. High calcined catalyst have less surface area, loss of boding and uneven distribution compared to low calcined temperature. At low calcination temperature, catalyst becomes weak. Modifier could affect the structure, stability, activity and surface area of synthesized catalyst. Modifiers decrease the sur- face area but improves the activity and stability of catalyst.

The characterization methods, such as: SEM, XRD, FTIR, TEM, TGA, PZC, TPR, and DRS of synthesized catalyst, gives the ideas of their structure, nature, dimensions, shape, composition, functional group, distribution, dispersion, mapping, surface area, pore volume, diameter, thermal stability, nucleation, stability and band gap energy of catalyst.

\section{Conclusion and Recommendations}

The present review is focused on the different catalyst preparation methods like wet impregnation, hydrothermal, precipitation, and sol-gel. Different methods have their own significant importance, but the challenge is to identify the selectivity of the method. In this area, there is a tremendous hope to do something new by means of catalyst modification. Catalyst modification is a very vast area in the synthesis of the catalyst. The main objective of catalyst modification is to enhance the catalytic efficiency, fast catalytic reaction, surface area, pore volume, catalyst stability, and reusability. There are various ways to do modification on the catalyst, discussed in details in this review. Apart from established modification techniques, some new modification techniques need to be developed, so that it can be easily and cost-effectively applied in industrial scale level. In this work, we also discussed the factors which are responsible for the synthesis of effective catalysts like $\mathrm{pH}$, temperature or modifier. Characterization of any catalyst is necessary to reveal the reason behind effective and successful catalyst. On the basis of that, we can optimize the synthesis method, modification techniques and other factors. Many characterizations already have been done by previous researchers, but in this work, only specific characterization was discussed for the synthesized catalyst.

\section{Acknowledgment}

This work was supported by Department of Science \& Technology- Science \& Engineering Research Board, File No. YSS/2014/000996, India. 


\section{References}

[1] Guo, Y., Xue, Q., Zhang, H., Wang, N., Chang, S., Wang, H., Pang, H., Chen, H. (2018). RSC Adv., 8, 80-90.

[2] Soltani, T., Lee, B.K., Hazard, J. (2016). $M a-$ ter., 316, 122-133.

[3] Ashokkumar, M., Cavalieri, F., Chemat, F., Okitsu, K., Sambandam, A., Yasui, K., Zisu, B. (2016). Handbook of Ultrasonics and Sonochemistry.

[4] Banerjee, S., Benjwal, P., Singh, M., Kar, K.K. (2018). Appl. Surf. Sci., 439, 560-568.

[5] Diya'uddeen, B.H., Rahim Pouran, S., Abdul Aziz, A.R., Daud, W.M.A.W. (2015). RSC Adv., 5, 68159-68168.

[6] Zhuang, H., Shan, S., Fang, C., Song, Y., Xue, X. (2018). Bioresources, 13, 4175-4186.

[7] Song, Y., Rong, C., Shang, J., Wang, Y., Zhang, Y., Yu, K. (2017). J. Chem. Technol. Biotechnol., 92, 2038-2049.

[8] Ogbiye, A.S., Omole, D.O., Ade-Balogun, K.D., Onakunle, O., Elemile, O.O. (2018). Cogent. Eng., 5, 1-15.

[9] Asaithambi, P., Matheswaran, M. (2016). Arab. J. Chem., 9, S981-S987.

[10] Carrasco-Díaz, M.R., Castillejos-López, E., Cerpa-Naranjo, A., Rojas-Cervantes, M.L. (2017). Microporous Mesoporous Mater., 237, 282-293.

[11] Ternes, T.A. (1998). Water Res., 32, 32453260 .

[12] Du, D., Shi, W., Wang, L., Zhang, J. (2017). Appl. Catal. B Environ., 200, 484-492.

[13] Lima, M.J., Silva, C.G., Silva, A.M.T., Lopes, J.C.B., Dias, M.M., Faria, J.L. (2017). Chem. Eng. J., 310, 342-351.

[14] Sun, X., Wang, C., Li, Y., Wang, W., Wei, J. (2015). Desalination, 355, 68-74.

[15] Mohammadi, S., Kargari, A., Sanaeepur, H., Abbassian, K., Najafi, A., Mofarrah, E. (2015). Desalin. Water Treat., 53, 2215-2234.

[16] Villegas, L.G.C., Mashhadi, N., Chen, M., Mukherjee, D., Taylor, K.E., Biswas, N. (2016). Curr. Pollut. Reports, 2, 157-167.

[17] Dang, T.H., Denat, A. (2018). $2^{\text {nd }}$ International conference on advanced materials (ICAM2017), IOP Conf. Series: Mater. Sci. Eng., 305, $1-10$

[18] Gao, P., Song, Y., Hao, M., Zhu, A., Yang, H., Yang, S. (2018). Sep. Purif. Technol., 201, 238-243.
[19] Gupta, D., Garg, A., (2018). React. Kinet. Mech. Catal., 124, 101-121.

[20] Chen, W., Yang, X., Huang, J., Zhu, Y., Zhou, Y., Yao, Y., Li, C. (2016). Electrochim. Acta, 200, 75-83.

[21] Sheng, Y., Sun, Y., Xu, J., Zhang, J., Han, Y.F. (2018). AIChE J., 64, 538-549.

[22] Vasilyeva, M.S., Rudnev, V.S., Zvereva, A.A., Ustinov, A.Y., Arefieva, O.D., Kuryavyi, V.G., Zverev, G.A. (2018). J. Photochem. Photobiol. A Chem., 356, 38-45.

[23] Dinarvand, M., Sohrabi, M., Royaee, S.J., Zeynali, V. (2017). Asia-Pacific J. Chem. Eng., 12, 631-639.

[24] Duan, F., Yang, Y., Li, Y., Cao, H., Wang, Y., Zhang, Y. (2014). J. Environ. Sci. (China), 26, 1171-1179.

[25] Nagabhushana, H., Saundalkar, S.S., Muralidhar, L., Nagabhushana, B.M. (2011). Chinese Chem. Lett., 22, 143-146.

[26] Wang, W.M., Song, J., Han, X. (2013). J. Hazard. Mater., 262, 412-419.

[27] Yu, L., Chen, J., Liang, Z., Xu, W., Chen, L., Ye, D. (2016). Sep. Purif. Technol., 171, 8087.

[28] Jinisha, R., Gandhimathi, R., Ramesh, S.T., Nidheesh, P.V., Velmathi, S. (2018). Chemospherem, 200, 446-454.

[29] Rostamizadeh, M., Jafarizad, A., Gharibian, S. (2018). Sep. Purif. Technol., 192, 340-347.

[30] Rashad, M.M., Ibrahim, A.A., Rayan, D.A., Sanad, M.M.S., Helmy, I.M. (2017). Environ. Nanotechnology, Monit. Manag., 8, 175-186.

[31] Muruganandam, L., Kumar, M.P.S., Jena, A., Gulla, S., Godhwani, B. (2017). IOP Conf. Ser. Mater. Sci. Eng., 263, 1-11.

[32] Sowinska, A., Pawlak, M., Mazurkiewicz, J., Pacholska, M. (2017). Water (Switzerland), 9, 1-14.

[33] A.R. Martins, A.B. Salviano, A.A.S. Oliveira, R. V. Mambrini, F.C.C. Moura, (2017) Environ. Sci. Pollut. Res. 24, 5991-6001.

[34] A. Jain, M. Kotwal, S. Khan, 1 (2016) Asian J. Chem. Pharm. Sci. 1-22.

[35] S. Krishnan, H. Rawindran, C.M. Sinnathambi, J.W. Lim, (2017) IOP Conf. Ser. Mater. Sci. Eng. 206, 1-11.

[36] M. Baerns (2004). Series: Springer Series in Chemical Physics, 75, 6221-6779.

[37] Alam, U., Khan, A., Bahnemann, D., Muneer, M. (2018). J. Colloid Interface Sci., 509, 6872 . 
[38] Chanderia, K., Kumar, S., Sharma, J., Ameta, R., Punjabi, P.B. (2017). Arab. J. Chem., 10, S205-S211.

[39] Córdoba, A., Saux, C., Pierella, L.B. (2017). Appl. Catal. A Gen., 544, 173-180.

[40] Dong, Q., Chen, Y., Wang, L., Ai, S., Ding, H. (2017). Appl. Surf. Sci., 426, 1133-1140.

[41] Karthikeyan, S., Pachamuthu, M.P., Isaacs, M.A., Kumar, S., Lee, A.F., Sekaran, G. (2016). Appl. Catal. B Environ., 199, 323-330.

[42] Lan, H., Wang, A., Liu, R., Liu, H., Qu, J. (2015). J. Hazard. Mater., 285, 167-172.

[43] Guo, S., Zhang, G., Guo, Y., Yu, J.C. (2013). Carbon, 60, 437-444.

[44] Wei, G.T., Fan, C.Y., Zhang, L.Y., Ye, R.C., Wei, T.Y., Tong, Z.F. (2012). Catal. Commun., 17, 184-188.

[45] Pachamuthu, M.P., Karthikeyan, S., Maheswari, R., Lee, A.F., Ramanathan, A. (2017). Appl. Surf. Sci., 393, 67-73.

[46] Liu, T., You, H., Chen, Q. (2009). J. Hazard. Mater., 162, 860-865.

[47] Noorjahan, M., Durga Kumari, V., Subrahmanyam, M., Panda, L. (2005). Appl. Catal. B Environ., 57, 291-298.

[48] Araña, J., González Díaz, O., Doña Rodríguez, J.M., Herrera Melián, J.A., Garriga i Cabo, C., Pérez Peña, J., Carmen Hidalgo, M., Navío-Santos, J.A. (2003). J. Mol. Catal. A Chem., 197, 157-171.

[49] Zhao, D., Yi, B.L., Zhang, H.M., Yu, H.M., Wang, L., Ma, Y.W., Xing, D.M. (2009). J. Power Sources, 190, 301-306.

[50] Dükkancı, M., Gündüz, G., Yılmaz, S., Prihod, R.V., Dükkanci, M, Gündüz, G., Yilmaz, S., Prihod'ko, R.V. (2010). J. Hazard. Mater., 181, 343-350.

[51] Gajović, A., Silva, A.M.T., Segundo, R.A., Šturm, S., Jančar, B., Čeh, M. (2011). Appl. Catal. B Environ., 103, 351-361.

[52] Wu, M., Yan, J.M., Zhao, M., Jiang, Q. (2012). Chempluschem, 77, 931-935.

[53] Chen, Y., Li, N., Zhang, Y., Zhang, L. (2014). J. Colloid Interface Sci., 422, 9-15.

[54] Zhang, X., Zhang, Y., Gao, L., Yu, H., Wei, Y. (2015). J. Colloid Interface Sci., 452, 24-32.

[55] An, J, Zhang, G., Zheng, R., Wang, P. (2016). J. Environ. Sci. (China), 48, 218-229.

[56] Han, C., Huang, G., Zhu, D., Hu, K. (2017). Mater. Chem. Phys., 200, 16-22.

[57] Bian, L., Liu, Y., Zhu, G., Yan, C., Zhang, J., Yuan, A. (2018). Ceram. Int., 44, 7580-7587.
[58] Diao, Y., Yan, Z., Guo, M., Wang, X. (2018). J. Hazard. Mater., 344, 829-838.

[59] He, Y., Bin Jiang, D., Jiang, D.Y., Chen, J., Zhang, Y.X. (2018). J. Hazard. Mater., 344, 230-240.

[60] Escribano, P.G., del Río, C., Morales, E., Aparicio, M., Mosa, J. (2018). J. Memb. Sci., 551, 136-144.

[61] Vinosha, P.A., Das, S.J., Annie Vinosha, P., Jerome Das, S. (2018). Mater. Today Proc., 5, 8662-8671.

[62] Rezgui, S., Amrane, A., Fourcade, F., Assadi, A., Monser, L., Adhoum, N. (2018). Appl. Catal. B Environ. 226, 346-359.

[63] Sharma, A., Dutta, R.K. (2018). J. Clean. Prod., 185, 464-475.

[64] García, J.C., Castellanos, M.P., Uscátegui, Á., Fernández, J., Pedroza, A.M., Daza, C.E. (2012). Univ. Sci., 17, 303-314.

[65] Ayoub, H., Roques-Carmes, T., Potier, O., Koubaissy, B., Pontvianne, S., Lenouvel, A., Guignard, C., Mousset, E., Poirot, H., Toufaily, J., Hamieh, T. (2018). Environ. Sci. Pollut. Res., 25, 34950-34967.

[66] Wang, N. (2017). RSC Adv., 7, 27619-27628.

[67] Mostafa Sadek, A. (2016). Am. J. Chem. Eng., 4, 1-8.

[68] Wang, N., Chen, J., Zhao, Q., Xu, H. (2017). RSC Adv., 7, 52524-52532.

[69] Wang, N., Hao, L., Chen, J., Zhao, Q., Xu, H. (2018). Environ. Sci. Pollut. Res., 25, 1248112490 .

[70] Barbosa, I.A., Zanatta, L.D., Espimpolo, D.M., da Silva, D.L., Nascimento, L.F., Zanardi, F.B., de Sousa Filho, P.C., Serra, O.A., Iamamoto, Y. (2017). Solid State Sci., 72,1420.

[71] Mena, I.F., Diaz, E., Rodriguez, J.J., Mohedano, A.F. (2017). Chem. Eng. J., 318, 153-160.

[72] Garrido-Ramírez, E.G., Marco, J.F., Escalona, N., Ureta-Zañartu, M.S. (2016). Microporous Mesoporous Mater., 225, 303-311.

[73] Idrissi, M., Miyah, Y., Benjelloun, Y., Chaouch, M. (2016). J. Mater. Environ. Sci., 7, 50-58.

[74] Doumic, L.I., Salierno, G., Ramos, C., Haure, P.M., Cassanello, M.C., Ayude, M.A. (2016). RSC Adv., 6, 46625-46633.

[75] Esteves, B.M., Rodrigues, C.S.D., Boaventura, R.A.R., Maldonado-Hódar, F.J., Madeira, L.M. (2016). J. Environ. Manage., 166, 193203. 
[76] Zhang, R., You, H., Wu, D. (2016). Desalin. Water Treat., 57, 12010-12018.

[77] Singh, L., Rekha, P., Chand, S. (2016). Sep. Purif. Technol., 170, 321-336.

[78] Sable, S.S., Ghute, P.P., Álvarez, P., Beltrán, F.J., Medina, F., Contreras, S. (2015). Catal. Today, 240, 46-54.

[79] di Luca, C., Ivorra, F., Massa, P., Fenoglio, R. (2015). Chem. Eng. J., 268, 280-289.

[80] Lu, M., Yao, Y., Gao, L., Mo, D., Lin, F., Lu, S. (2015). Water. Air. Soil Pollut., 226, 87-90.

[81] Pan, W., Zhang, G., Zheng, T., Wang, P. (2015). RSC Adv., 5, 27043-27051.

[82] Xie, F., Zhong, J., Wang, L., Wang, K., Hua, D.X. (2015). IOP Conf. Ser. Mater. Sci. Eng., 87, 1-7.

[83] Ersöz, G. (2014). Appl. Catal. B Environ., 147, $353-358$.

[84] Zaidill Azizan, M.A., Hassan, H., Faraziehan, S., Abu Hassan, N. (2014). Appl. Mech. Mater., 661, 29-33.

[85] Xiong, W., Liu, D.R., Yuan, G.Y., Wei, Q., Sen Dang, Q., Feng, J., Xu, B. (2014). Adv. Mater. Res., 881-883, 279-282.

[86] Ramli, R.M., Kait, C.F., Omar, A.A. (2014). Adv. Mater. Res., 917, 160-167.

[87] Azmi, N.H.M., Vadivelu, V.M., Hameed, B.H. (2014). Desalin. Water Treat., 52, 5583-5593.

[88] Yang, C., Wang, D., Tang, Q. (2014). J. Taiwan Inst. Chem. Eng., 45, 2584-2589.

[89] Bayat, M., Sohrabi, M., Royaee, S.J. (2012). J. Ind. Eng. Chem., 18, 957-962.

[90] Xu, J.Q., Guo, F., Zou, S.S., Quan, X.J. (2011). Mater. Sci. Forum, 694, 640-644.

[91] Xu, L., Wang, J. (2012). Environ. Sci. Technol., 46, 10145-10153.

[92] Dhaouadi, A., Adhoum, N. (2010). Appl. Catal. B Environ., 97, 227-235.

[93] Shukla, P., Wang, S., Sun, H., Ang, H.M., Tadé, M. (2010). Chem. Eng. J., 164, 255-260.

[94] Gan, G., Liu, J., Zhu, Z., Yang, Z., Zhang, C., Hou, X. (2017). Chemosphere, 168, 254-263.

[95] Wan, Z., Wang, J. (2017). J. Hazard. Mater., 324, 653-664.

[96] Jusoh, R., Jalil, A.A., Triwahyono, S., Idris, A., Noordin, M.Y. (2015). Sep. Purif. Technol., 149, 55-64.

[97] Haber, J., Block, J., H., Delmon, B. (1995). Pure Appl. Chem., 67, 1257-1306.

[98] Bian, L., Liu, Y., Zhu, G., Yan, C., Zhang, J., Yuan, A. (2018). Ceram. Int., 44, 7580-7587.
[99] Fei, B.L., Zhong, J.K., Deng, N.P., Wang, J.H., Liu, Q.B., Li, Y.G., Mei, X. (2018). Chemosphere, 197, 241-250.

[100] Yu, D., Ni, H., Wang, L., Wu, M., Yang, X. (2018). J. Clean. Prod., 186, 146-154.

[101] Xiao, C., Li, J., Zhang, G. (2018). J. Clean. Prod., 180, 550-559.

[102] Edla, R., Tonezzer, A., Orlandi, M., Patel, N., Fernandes, R., Bazzanella, N., Date, K., Kothari, D.C., Miotello, A. (2017). Appl. Catal. B Environ., 219, 401-411.

[103] Jaramillo-Páez, C., Navío, J.A., Hidalgo, M.C., Bouziani, A., El Azzouzi, M. (2017). J. Photochem. Photobiol. A Chem., 332, 521533.

[104] Zhao, Z., Zhou, X., Liu, Z. (2015). Water Air Soil Pollut., 228, 463, 1-11.

[105] Liang, C., Liu, Y., Li, K., Wen, J., Xing, S, Ma, Z., Wu, Y. (2017). Sep. Purif. Technol., 188, 105-111.

[106] Ma, P., Yu, Y., Xie, J., Fu, Z. (2017). Adv. Powder Technol., 28, 2797-2804.

[107] Ojha, D.P., Joshi, M.K., Kim, H.J. (2017). Ceram. Int., 43, 1290-1297.

[108] To, T., Phan, N., Nikoloski, A.N., Bahri, P.A., Li, D. (2017). RSC. $A d v .$, 8, 36181-36190.

[109] Sun, H., Dong, B., Song, L., Du, J., Gao, R., Su, G., Cao, L. (2017). J. Photochem. Photobiol. A Chem., 334, 20-25.

[110] Dhiman, M., Sharma, R., Kumar, V., Singhal, S. (2016). Ceram. Int., 42, 12594-12605.

[111] Wang, Z., Fan, Y., Wu, R., Huo, Y., Wu, H., Wang, F., Xu, X. (2018). RSC Adv., 8, 51805188.

[112] Turki, A., Kochkar, H., Berhault, G., Ghorbel, A., (2010). P-Hydroxybenzoic Acid Degradation by $\mathrm{Fe} / \mathrm{Pd}$-HNT Catalysts with in Situ Generated Hydrogen Peroxide, Elsevier B.V.

[113] Wu, D., Long, M., Chen, C., Wu, Y., Cai, W., Zhou, J., Ding, D. (2009). Water Sci. Technol., 59, 565-571.

[114] Bolova, E., Gunduz, G., Dukkanci, M., Yilmaz, S., Yaman, Y.C. (2011). Int. J. Chem. React. Eng., 9, 1-20.

[115] Li, D., Pan, C., Shi, R., Zhu, Y. (2011). CrystEngComm, 13, 6688.

[116] Xu, Z., Zhang, M., Wu, J., Liang, J., Zhou, L., Lü, B. (2013). Water Sci. Technol., 68, 21782185.

[117] Vu, T.A., Le, G.H., Dao, C.D., Dang, L.Q., Nguyen, K.T., Dang, P.T., Tran, H.T.K., Duong, Q.T., Nguyen, T.V., Lee, G.D. (2014). RSC Adv., 4, 41185-41194. 
[118] Hu, Z.T., Da Oh, W., Liu, Y., Yang, E.H., Lim, T.T. (2018). J. Colloid Interface Sci., 509, 502-514.

[119] Ren, M., Qian, X., Fang, M., Yue, D., Zhao, Y. (2018). Res. Chem. Intermed., 44, 4103-4117.

[120] Ridruejo, C., Alcaide, F., Álvarez, G., Brillas, E., Sirés, I. (2018). J. Electroanal. Chem., 808, 364-371.

[121] Zhang, X., Dong, J., Hao, Z., Cai, W., Wang, F. (2018). Trans. Tianjin Univ., 24, 361-369.

[122] Zhang, H., Xue, G., Chen, H., Li, X. (2018). Chemosphere, 191, 64-71.

[123] Zhang, Z., Guo, Y., Wang, Q., Louis, B., Qi, F. (2017). Comptes Rendus Chim., 20, 87-95.

[124] Zhang, L., Xu, D., Hu, C., Shi, Y. (2017). Appl. Catal. B Environ., 207, 9-16.

[125] Xie, Z., Wang, C., Yin, L. (2017). J. Catal., 353, 11-18.

[126] Wang, X., Dou, L., Yang, L., Yu, J., Ding, B. (2017). J. Hazard. Mater., 324, 203-212.

[127] Tang, J., Wang, J. (2017). RSC Adv., 7, 50829-50837.

[128] Ramesh, M., Rao, M.P., Rossignol, F., Nagaraja, H.S. (2017). Water Sci. Technol., 76, 1652-1665.

[129] Kim, E.J., Oh, D., Lee, C.S., Gong, J., Kim, J., Chang, Y.S. (2017). Catal. Today, 282, 71-76.

[130] Li, J, Zhang, S., Chen, Y., Liu, T., Liu, C., Zhang, X., Yi, M., Chu, Z., Han, X. (2017). RSC Adv., 7, 29051-29057.

[131] Chai, F., Li, K., Song, C., Guo, X. (2016). J. Colloid Interface Sci., 475, 119-125.

[132] Li, S., Zhang, G., Zhang, W., Zheng, H., Zhu, W, Sun, N., Zheng, Y., Wang, P. (2017). Chem. Eng. J., 326, 756-764.

[133] Li, S., Zhang, G., Zheng, H., Wang, N., Zheng, Y., Wang, P. (2016). RSC Adv., 6, 8243982446.

[134] Wang, R., Liu, X., Wu, R., Yu, B., Li, H., Zhang, X., Xie, J., Yang, S.-T. (2016). RSC Adv., 6, 8594-8600.

[135] Yang, X., Sun, H., Zhang, L., Zhao, L., Lian, J., Jiang, Q. (2016). Sci. Rep., 6, 1-12.

[136] Mao, Y., Zou, H., Wang, Q., Huang, C. (2016). Sci. China Chem., 59, 903-909.

[137] Lyu, L., Zhang, L., Hu, C., Yang, M. (2016). J. Mater. Chem. A, 4, 8610-8619.

[138] Kong, L., Zhou, X., Yao, Y., Jian, P., Diao, G. (2016). Environ. Technol. (United Kingdom), 37, 422-429.

[139] Hao, S.M., Qu, J., Zhu, Z.S., Zhang, X.Y., Wang, Q.Q., Yu, Z.Z. (2016). Adv. Funct. Mater., 26, 7334-7342.
[140] Fang, D., Yu, Y., Xu, Z., Liang, J, Zhou, L. (2016). Mater. Chem. Phys., 171, 386-393.

[141] Zhou, M., Li, W., Du, Y., Kong, D., Wang, Z., Meng, Y., Sun, X., Yan, T., Kong, D., You, J. (2016). J. Nanoparticle Res.,18, 346, 1-15.

[142] Lyu, L., Zhang, L., Hu, C. (2016). Environ. Sci. Nano, 3, 1483-1492.

[143] Edla, R., Patel, N., Orlandi, M., Bazzanella, N., Bello, V., Maurizio, C., Mattei, G., Mazzoldi, P., Miotello, A. (2015). Appl. Catal. B Environ., 166-167, 475-484.

[144] Jia, Y., Wu, C., Kim, D.H, Lee, B.W., Rhee, S.J., Park, Y.C., Kim, C.S., Wang, Q.J., Liu, C. (2018). Chem. Eng. J., 337, 709-721.

[145] Mahy, J.G, Tasseroul, L., Herlitschke, M., Hermann, R.P., Lambert, S.D. (2016). Mater. Today Proc., 3, 464-469.

[146] Uma, K., Arjun, N., Pan, G.T., Yang, T.C.K. (2017). Appl. Surf. Sci., 425, 377-383.

[147] Nurhayati, E., Yang, H., Chen, C., Liu, C., Juang, Y., Huang, C., chang Hu, C. (2016). Int. J. Electrochem. Sci., 11, 3615-3632.

[148] Wang, L., Yu, Z., Peng, Z., Chen, Y., Xiang, G., Liu, Q., Liu, Y., Chen, D. (2016). Russ. J. Phys. Chem. A, 90, 777-782.

[149] Martínez, F., Molina, R., Pariente, M.I., Siles, J.A, Melero, J.A. (2017). Catal. Today, 280, 176-183.

[150] Soltani, T., Lee, B.K. (2017). Chem. Eng. J., 313, 1258-1268.

[151] Su, L, Li, K., Zhang, H., Fan, M., Ying, D., Sun, T., Wang, Y., Jia, J. (2017). Water Res., $120,1-11$.

[152] Ribeiro, R.S., Frontistis, Z., Mantzavinos, D., Venieri, D., Antonopoulou, M., Konstantinou, I., Silva, A.M.T., Faria, J.L., Gomes, H.T. (2016). Appl. Catal. B Environ., 199, 170-186.

[153] Soltani, T., Lee, B.K. (2016). J. Mol. Catal. A Chem., 425, 199-207.

[154] Cheng, C.K., Kong, Z.Y., Khan, M.R. (2015). Water. Air. Soil Pollut., 226, 1-12.

[155] Feng, J., Wong, R.S.K., Hu, X., Yue, P.L. (2004). Catal. Today, 98, 441-446.

[156] Vinosha, P.A., Xavier, B., Krishnan, S., Das, S.J. (2018). Mater. Res. Bull., 101, 190-198.

[157] Vinosha, P.A., Xavier, B., Anceila, D., Das, S.J. (2018). Optik (Stuttg), 157, 441-448.

[158] Vinosha, P.A., Xavier, B., Ashwini, A., Ansel Mely, L., Das, S.J. (2017). Optik (Stuttg)., 137, 244-253.

[159] Xu, H.-Y., Wang, Y., Shi, T.-N., Zhao, H., Tan, Q., Zhao, B.-C., He, X.-L., Qi, S.-Y. (2018). Front. Mater. Sci., 12, 34-44. 
[160] Zuorro, A., Lavecchia, R., Monaco, M.M., Iervolino, G., Vaiano, V. (2017). Catalysts, $9(8), 645$.

[161] Bai, J., Liu, Y., Yin, X., Duan, H., Ma, J. (2017). Appl. Surf. Sci., 416, 45-50.

[162] Barbosa, I.A., Zanatta, L.D., Espimpolo, D.M., da Silva, D.L., Nascimento, L.F., Zanardi, F.B., de Sousa Filho, P.C., Serra, O.A., Iamamoto, Y. (2017). Solid State Sci., 72, 14-20.

[163] Fida, H., Zhang, G., Guo, S., Naeem, A. (2017). J. Colloid Interface Sci., 490, 859-868.

[164] Gogoi, A., Navgire, M., Sarma, K.C., Gogoi, P. (2017). Chem. Eng. J., 311, 153-162.

[165] Liu, Y., Zhang, G., Chong, S., Zhang, N., Chang, H., Huang, T., Fang, S. (2017). J. Environ. Manage., 192, 150-155.

[166] Shen, Y., Zhou, Y., Zhang, Z., Xiao, K. (2017). J. Ind. Eng. Chem., 52, 153-161.

[167] Tian, X., Liu, Y., Chi, W., Wang, Y., Yue, X., Huang, Q., Yu, C. (2017). Water. Air. Soil Pollut., 228, 1-12.

[168] Wan, D., Wang, G., Li, W., Wei, X. (2017). Appl. Surf. Sci., 413, 398-407.

[169] Ya, V., Martin, N., Chou, Y.H., Chen, Y.M., Choo, K.H., Chen, S.S., Li, C.W. (2018). J. Taiwan Inst. Chem. Eng., 83, 107-114.

[170] Xu, R., Huang, X., Li, H., Su, M., Chen, D. (2018). IOP Conf. Ser. Earth Environ. Sci., 111, 1-6.

[171] Tiya-Djowe, A., Ruth, N., Kamgang-Youbi, G., Acayanka, E., Laminsi, S., Gaigneaux, E.M. (2018). Microporous Mesoporous Mater., 255, $148-155$.

[172] Shi, X., Tian, A., You, J., Yang, H., Wang, Y., Xue, X. (2018). J. Hazard. Mater., 353, 182189.

[173] Santos, A.P.F., Souza, B.M., Silva, T.F.C.V., Cavalcante, R.P., Oliveira, S.C., Machulek, A., Boaventura, R.A.R., Vilar, V.J.P. (2018). Environ. Sci. Pollut. Res., 25, 1-13.

[174] He, H., Zhang, X., Yang, C., Zeng, G., Li, H., Chen, Y. (2018). J. Environ. Eng., 144, 04018041.

[175] Zhang, H., Liu, J., Ou, C., Faheem, C., Shen, J., Yu, H.., Jiao, Z., Han, W., Sun, X., Li, J., Wang, L. (2017). J. Environ. Sci. (China). 53, $1-8$.

[176] Takayanagi, A., Kobayashi, M., Kawase, Y. (2017). Environ. Sci. Pollut. Res., 24, 80878097.

[177] Vilar, V.J.P., Silva, T.F.C.V., Santos, M.A.N., Fonseca, A., Saraiva, I., Boaventura, R.A.R. (2012). Sol. Energy, 86, 3301-3315.
[178] Nogueira, A.A., Souza, B.M., Dezotti, M.W.C., Boaventura, R.A.R., Vilar, V.J.P. (2017). J. Photochem. Photobiol. A Chem., 345, 109123.

[179] Glugoski, L.P., de Jesus Cubas, P., Fujiwara, S.T. (2017). Environ. Sci. Pollut. Res. 24, 6143-6150.

[180] Frade, V.M.F., Converti, A., Al Arni, S., Silva, M.F., Palma, M.S.A. (2017). Chem. Eng. Technol., 40, 663-669.

[181] Emin, M., Alver, A, Karataş, M. (2017). Journal name??, 90, 21241.

[182] Xie, X., Hu, Y., Cheng, H. (2016). Water Res., $89,59-67$.

[183] Wang, P., Wang, L., Sun, Q., Qiu, S., Liu, Y., Zhang, X, Liu, X., Zheng, L. (2016). Mater. Lett., 183, 61-64.

[184] Valero-Luna, C., Palomares-Sanchéz, S.A., Ruíz, F. (2016). Catal. Today, 266, 110-119.

[185] Su, C., Li, W., Liu, X., Huang, X., Yu, X. (2016). Front. Environ. Sci. Eng., 10, 37-45.

[186] Peng, S., Zhang, W., He, J., Yang, X., Wang, D., Zeng, G. (2016). J. Environ. Sci. (China), $41,16-23$.

[187] Expósito, A.J., Monteagudo, J.M., Díaz, I., Durán, A. (2016). Chem. Eng. J., 306, 12031211.

[188] W., Wan, D., Wang, G., Chen, K., Hu, Q., Lu, L. (2016). Korean J. Chem. Eng., 33, 15571564 .

[189] Kim Phuong, N.T., wook Beak, M., Huy, B.T., Lee, Y.I. (2016). Chemosphere, 146, 51-59.

[190] Nadejde, C., Neamtu, M., Hodoroaba, V.D., Schneider, R.J., Paul, A., Ababei, G., Panne, U. (2015). J. Nanoparticle Res., 17, 1-10.

[191] Ealias, A.M., Jose, J.V., Saravanakumar, M.P. (2016). Environ. Sci. Pollut. Res., 23, 21416-21430.

[192] Kakarla, P.K., Andrews, T., Greenberg, R.S., Zervas, D.S. (2002). Remediation, 12, 23-36.

[193] Reyes, I.A., Patiño, F., Flores, M.U., Narayanan, J., Calderón, H., Pandiyan, T. (2013). J. Mex. Chem. Soc., 57, 96-104.

[194] Barbusiński, K. (2014). Polish J. Environ. Stud. 14(3), 281-285.

[195] Banuelos, J.A., Garcia-Rodriguez, O., Rodriguez-Valadez, F.J., Godinez, L.A. (2015). J. Electrochem. Soc., 162, E154-E159.

[196] Rahman, W.U., Khan, M.D., Khan, M.Z., Halder, G. (2018). J. Environ. Chem. Eng., 6, 2957-2964.

[197] Ganesan, R., Thanasekaran, K. (2011). Int. J. Environ. Sci., 1, 1168-1176. 
[198] Lewis, S., Lynch, A., Bachas, L., Hampson, S., Ormsbee, L., Bhattacharyya, D. (2009). Environ. Eng. Sci., 26, 849-859.

[199] Lee, S., Oh, J., Park, Y. (2006). Bull. Chem. Soc., 27, 489-494.

[200] Zhao, X., Dong, Y., Cheng, B., Kang, W. (2013). Int. J. Photoenergy, 2009, 1-9.

[201] Pradisty, N.A., Sihombing, R., Howe, R.F., Krisnandi, Y.K. (2017). Makara J. Sci., 21, 25-33.

[202] Tony, M.A., Zhao, Y.Q., Purcell, P.J., ElSherbiny, M.F. (2009). J. Environ. Sci. Heal. Part A Toxic/Hazardous Subst. Environ. Eng., 44, 488-493.

[203] Lee, S.Y., Park, S.J. (2013). J. Ind. Eng. Chem., 19, 1761-1769.

[204] Kurian, M., Nair, D.S. (2015). J. Water Process Eng., 8, e37-e49.

[205] Lee, H., Choi, J., Lee, S., Yun, S.T., Lee, C., Lee, J. (2013). Appl. Catal. B Environ., 138139, 311-317.

[206] Huang, Z., Wu, P., Li, H., Li, W., Zhu, Y., Zhu, N. (2014). RSC Adv., 4, 6500.

[207] Liang, C., Zhao, W., Song, Z., Xing, S. (2017). RSC Adv., 7, 35257-35264.

[208] Huang, H., Shan, W., Yang, S., Zhang, J. (2014). Catal. Sci. Technol., 4, 3611-3614.

[209] Yong, E.L., Lin, Y.P. (2016). RSC Adv., 6, 18587-18595.

[210] Fan, Y., Ma, W., He, J., Du, Y. (2017). RSC Adv., 7, 36193-36200.

[211] Chen, Y.F., Lee, C.Y., Yeng, M.Y., Chiu, H.T. (2003). J. Cryst. Growth, 247, 363-370.

[212] Li, G., Liu, Z.Q., Lu, J., Wang, L., Zhang, Z. (2009). Appl. Surf. Sci., 255, 7323-7328.

[213] Park, G.C., Seo, T.Y., Park, C.H., Lim, J.H., Joo, J. (2017). Ind. Eng. Chem. Res., 56, 8235-8240.

[214] Edwards, J.K., Pritchard, J., Piccinini, M., Shaw, G., He, Q., Carley, A.F., Kiely, C.J., Hutchings, G.J. (2012). J. Catal., 292, 227238.

[215] Wang, Y., Yu, Y., Deng, C., Wang, J., Zhang, B.-T. (2015). RSC Adv., 5, 103989-103998.

[216] Alipour, Z., Rezaei, M., Meshkani, F. (2014). Fuel, 129, 197-203.

[217] Parvin, T., Keerthiraj, N., Ibrahim, I.A., Phanichphant, S., Byrappa, K. (2012). Int. J. Photoenergy., 2012, 1-8.

[218] López-Ramón, M.V., Álvarez, M.A., MorenoCastilla, C., Fontecha-cámara, M.A., YebraRodríguez, Á., Bailón-García, E. (2018). J. Colloid Interface Sci., 511, 193-202.
[219] Chaudhuri, R.G., Paria, S., Ghosh Chaudhuri, R., Paria, S. (2012). Chem. Rev., 112, 2373-2433.

[220] Zhou, C., Sun, L., Zhang, A., Wu, X., Ma, C., $\mathrm{Su}, \mathrm{S} ., \mathrm{Hu}, \mathrm{S}$. (2015). Chemosphere, 125, 1624.

[221] El-salamony, R.A. (2017). J. Sol-Gel Sci. Technol., 18, 102-112.

[222] Gobara, H., El-salamony, R., Mohamed, D., Mishrif, M., Moustafa, Y., Gendy, T. (2014). Chem. Mater. Res., 6, 63-82.

[223] Wang, J., Liu, C., Tong, L., Li, J., Luo, R., Qi, J., Li, Y., Wang, L. (2015). RSC Adv., 5, 69593-69605.

[224] Kaur, G., Singh, B., Singh, P., Kaur, M., Buttar, K.K., Singh, K., Thakur, A., Bala, R., Kumar, M., Kumar, A. (2016). RSC Adv., 6, 99120-99128.

[225] Banerjee, S., Benjwal, P., Singh, M., Kar, K.K. (2018). Appl. Surf. Sci., 439, 560-568.

[226] Yang, Z., Yu, A., Shan, C., Gao, G., Pan, B. (2018). Water Res., 137, 37-46.

[227] Rajoriya, S., Bargole, S., George, S., Kumar, V., Gogate, P.R. (2019). Sep. Purif. Technol., 209, 254-269.

[228] Kalam, A., Al-Sehemi, A.G., Assiri, M., Du, G., Ahmad, T., Ahmad, I., Pannipara, M. (2018). Results Phys., 8, 1046-1053.

[229] Tyagi, M., Rana, A., Kumari, S., Jagadevan, S. (2018). J. Clean. Prod., 178, 398-407.

[230] Xu, S., Zhu, H., Cao, W., Wen, Z., Wang, J., François-Xavier, C.P., Wintgens, T. (2018). Appl. Catal. B Environ., 234, 223-233.

[231] Wang, Q., Liang, S., Zhang, G., Su, R., Yang, C., Xu, P., Wang, P. (2019). Sep. Purif. Technol., 209, 270-278.

[232] Wang, B., Zhang, Q., Hong, J., Li, L. (2018). Process Saf. Environ. Prot., 119, 75-86.

[233] Samaddar, P., Ok, Y.S., Kim, K.H., Kwon, E.E., Tsang, D.C.W. (2018). J. Clean. Prod., 197, 1190-1209.

[234] Abukhadra, M.R., Rabia, M., Shaban, M., Verpoort, F. (2018). Adv. Powder Technol., 29, 2501-2511.

[235] Kalapathy, U., Proctor, A., Shultz, J. (2000). J. Chem. Technol. Biotechnol. 468, 464-468.

[236] Yu, G., Lyu, L., Zhang, F., Yan, D., Cao, W., Hu, C. (2018). RSC Adv., 8, 3312-3320.

[237] Mohamed, F., Abukhadra, M.R., Shaban, M. (2018). Sci. Total Environ., 640-641, 352363.

[238] Dong, C., Lu, J., Qiu, B., Shen, B., Xing, M., Zhang, J. (2018). Appl. Catal. B Environ., 222, 146-156. 
[239] Liu, N., Huang, W., Zhang, X., Tang, L., Wang, L., Wang, Y., Wu, M. (2018). Appl. Catal. B Environ., 221, 119-128.

[240] He, Z., Xia, D., Huang, Y., Tan, X., He, C., Hu, L., He, H., Zeng, J., Xu, W., Shu, D. (2018). J. Hazard. Mater., 344, 1198-1208.

[241] Gu, L., Zhu, N., Guo, H., Huang, S., Lou, Z., Yuan, H. (2013). J. Hazard. Mater., 246-247, $145-153$.

[242] Tursi, A., Beneduci, A., Chidichimo, F., De Vietro, N., Chidichimo, G. (2018). Chemosphere, 201, 530-539.

[243] Zhu, F., Ma, S., Liu, T., Deng, X. (2018). J. Clean. Prod., 174, 184-190.

[244] Nadejde, C., Neamtu, M., Hodoroaba, V., Schneider, R.J., Paul, A., Ababei, G., Panne, U. (2015). Applied Catal. B, Environ., 176177, 667-677.

[245] Rani, M., Shanker, U. (2018). J. Colloid Interface Sci., 530, 16-28.

[246] Chen, H., Lu, Q., He, K., Liu, M., Zhang, Y., Yao, S. (2018). Sensors Actuators, B Chem., 260, 908-917.

[247] Garcia, J., Gomes, H.T., Serp, P., Kalck, P., Figueiredo, J.L., Faria, J.L. (2005). Catal. Today, 103, 101-109.

[248] Yehia, F.Z., Eshaq, G., Rabie, A.M., Mady, A.H., Elmetwally, A.E. (2015). Egypt. J. Pet., $24,13-18$.

[249] Hou, X., Huang, X., Li, M., Zhang, Y., Yuan, S., Ai, Z., Zhao, J., Zhang, L. (2018). Chem. Eng. J., 348, 255-262.

[250] Cao, Z., Wen, X., Chen, P., Yang, F., Ou, X, Wang, S. (2018). Colloids Surfaces A, 549, 94104.

[251] Moura, C.C., Helena, M., Costa, R.C.C., Fabris, D., Ardisson, D., Macedo, W.A.A., Lago, R.M. (2005). Chemosphere, 60, 11181123.

[252] Donadelli, J.A., García Einschlag, F.S., Laurenti, E., Magnacca, G., Carlos, L. (2018). Colloids Surfaces B Biointerfaces, 161, 654-661.

[253] Wang, J., Liu, C., Hussain, I., Li, C., Li, J., Sun, X., Shen, J., Han, W., Wang, L. (2016). RSC Adv., 6, 54623-54635.

[254] Cotto-Maldonado, M.C. (2012). PhD Dissertation. Universidad del Turabo, 1-255.

[255] Diaz, J.L., Tuesta, D., Silva, A.M.T., Faria, J.L., Gomes, H.T. (2018). Chem. Eng. J., 347, 963-971.

[256] An, J., Zhu, L., Wang, N., Song, Z., Yang, Z., Du, D., Tang, H. (2013). Chem. Eng. J., 219, 225-237.
[257] Xiong, Q., Zhou, M., Yang, H., Liu, M., Wang, T., Dong, Y., Hou, H. (2018). ACS Sustain. Chem. Eng., 6, 872-881.

[258] Cao, J., Xiong, Z., Lai, B. (2018). Chem. Eng. J., 343, 492-499.

[259] Hogland, W., Marques, M. (2016). Chem. Eng. J., 219, 499-511.

[260] Ozinger, N., 1997. Weitkamp (eds.): Handbook of Heterogeneous Catalysis, Wiley-VCH, Weinheim, 1-107.

[261] Rezende, C.C., Neto, J.L., Silva, A.C., Lima, V.M., Pereira, M.C., Oliveira, L.C.A. (2012). Catal. Commun., 26, 209-213.

[262] Jia, X., Dai, R., Sun, Y., Song, H., Wu, X. (2015). J. Mater. Sci. Mater. Electron., 27, 3791-3798.

[263] Casas, J.A., Rodriguez, J.J., Bautista, P., Mohedano, A.F., Mene, N. (2010). Catal. Today, 151, 148-152.

[264] Bansal, P., Verma, A., Talwar, S. (2018). Chem. Eng. J., 349, 838-848.

[265] Ma, T., Zhang, L., Xi, B., Xiong, Y., Yu, P., Li, G., Li, J., Zhao, C. (2018). Ecol. Eng., 110, 192-203.

[266] Sekaran, G., Karthikeyan, S., Ramani, K., Ravindran, B., Gnanamani, A., Mandal, A.B. (2011). Environ. Chem. Lett., 9, 499-504.

[267] Banić, N., Abramović, B., Krstić, J., Šojić, D., Lončarević, D., Cherkezova-Zheleva, Z., Guzsvány, V. (2011). Appl. Catal. B Environ., 107, 363-371.

[268] Cao, C.-Y., Meng, L.-K., Zhao, Y.-H. (2013). Toxicol. Environ. Chem., 95, 747-756.

[269] Yingcai, W., Shuai, S., Can, W., Shuai, S.F. (2018). Environ. Prot. Eng., 44, 132-144.

[270] May-Marrufo, A.A., Mendez-Novelo, R.I., Barceló-Quintal, I.D., Solís-Correa, H.E., Giacoman-Vallejos, G. (2017). J. Environ. Prot. (Irvine,. Calif)., 08, 524-539.

[271] Duarte, F., Morais, V., Maldonado-hódar, F.J., Madeira, L.M. (2013). Chem. Eng. J., 232, 34-41.

[272] Rostamizadeh, M., Jafarizad, A., Gharibian, S. (2018). Sep. Purif. Technol., 192, 340-347.

[273] Shah, J., Jan, M.R., Khitab, F. (2018). Process Saf. Environ. Prot., 116, 149-158.

[274] Vanamudan, A., Sadhu, M., Pamidimukkala, P.S. (2018). J. Taiwan Inst. Chem. Eng., 85, 74-82.

[275] Nešic, J., Manojlovic, D.D., Jovic, M., Dojcinovic, B.P., Vulic, P.J., Krstic, J., Roglic, G.M. (2014). J. Serbian Chem. Soc., 79, 977-991. 
[276] Rashwan, W.E., Fathy, N.A., Elkhouly, S.M. (2018). J. Taiwan Inst. Chem. Eng., 88, 234242.

[277] Xue, F., Yang, S.-T., Jin, X., Li, T., Wang, R., Liu, X., Bai, Y., Chen, L., Ming, Z., Yang, H. (2016). Micro Nano Lett., 11, 675-679.

[278] Chen, H., Motuzas, J., Martens, W., Diniz da Costa, J.C. (2018). Sep. Purif. Technol., 205, 293-301.

[279] Barbosa, I.A., Zanatta, L.D., Espimpolo, D.M., Silva, D.L., Nascimento, L.F., Zanardi, F.B., Filho, P.C.D.S., Serra, O.A., Iamamoto, Y. (2017). Solid State Sci., 72, 14-20.

[280] Hu, L.X., Xu, D.D., Zou, L.P., Yuan, H., Hu, X. (2015). Wuli Huaxue Xuebao/ Acta Phys. Chim. Sin., 31, 771-782.

[281] Molla, A., Li, Y., Khandelwal, M., Mandal, B., Kang, S.G., Hur, S.H., Chung, J.S. (2018). J. Environ. Chem. Eng., 6, 2616-2626.

[282] Ayodele, O.B., Lim, J.K., Hameed, B.H. (2012). Appl. Catal. A Gen., 413, 301-309.
[283] Wang, H., Jiang, H., Wang, S., Shi, W., He, J., Liu, H., Huang, Y. (2014). RSC Adv., 4, 45809-45815.

[284] Shi, P., Su, R., Zhu, S., Zhu, M., Li, D., Xu, S. (2012). J. hazard. Mater., 229, 331-339.

[285] Istadi, I., Anggoro, D.D., Amin, N.A.S., Ling, D.H.W. (2011), Bull. Chem. React. Eng. Catal., 6 (2), 129-136.

[286] Variava, M.F., Church, T.L., Harris, A.T. (2012). Appl. Catal. B, 123, 200-207.

[287] Liu, T., You, H., Chen, Q. (2009). J. Hazard. Mater., 162, 860-865.

[288] Noorjahan, M., Kumari, V.D., Subrahmanyam, M., Panda, L. (2005). Appl. Catal. B Env., 57, 291-298.

[289] Iranmahboob, J., Hill, D.O., Toghiani, H. (2001). Appl. Surf. Sci., 185, 72-78. 